\title{
Search for anomalous couplings in the $W t b$ vertex from the measurement of double differential angular decay rates of single top quarks produced in the $t$-channel with the ATLAS detector
}

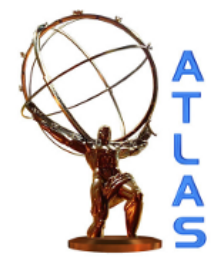

\section{The ATLAS collaboration}

\section{E-mail: atlas.publications@cern.ch}

ABSTRACT: The electroweak production and subsequent decay of single top quarks is determined by the properties of the $W t b$ vertex. This vertex can be described by the complex parameters of an effective Lagrangian. An analysis of angular distributions of the decay products of single top quarks produced in the $t$-channel constrains these parameters simultaneously. The analysis described in this paper uses $4.6 \mathrm{fb}^{-1}$ of proton-proton collision data at $\sqrt{s}=7 \mathrm{TeV}$ collected with the ATLAS detector at the LHC. Two parameters are measured simultaneously in this analysis. The fraction $f_{1}$ of decays containing transversely polarised $W$ bosons is measured to be $0.37 \pm 0.07$ (stat. $\oplus$ syst.). The phase $\delta_{-}$between amplitudes for transversely and longitudinally polarised $W$ bosons recoiling against lefthanded $b$-quarks is measured to be $-0.014 \pi \pm 0.036 \pi$ (stat. $\oplus$ syst.). The correlation in the measurement of these parameters is 0.15 . These values result in two-dimensional limits at the $95 \%$ confidence level on the ratio of the complex coupling parameters $g_{\mathrm{R}}$ and $V_{\mathrm{L}}$, yielding $\operatorname{Re}\left[g_{\mathrm{R}} / V_{\mathrm{L}}\right] \in[-0.36,0.10]$ and $\operatorname{Im}\left[g_{\mathrm{R}} / V_{\mathrm{L}}\right] \in[-0.17,0.23]$ with a correlation of 0.11 . The results are in good agreement with the predictions of the Standard Model.

KEYWORDS: Hadron-Hadron scattering

ARXIV EPRINT: 1510.03764 


\section{Contents}

1 Introduction 1

2 Measurement definition 3

3 The ATLAS detector 6

4 Data and simulated samples $\quad 7$

5 Object definitions and event selection $\quad 8$

6 Background estimation and event yields 10

7 Analysis method 11

8 Sources of systematic uncertainty $\quad 17$

$\begin{array}{lll}8.1 & \text { Object modelling } & 17\end{array}$

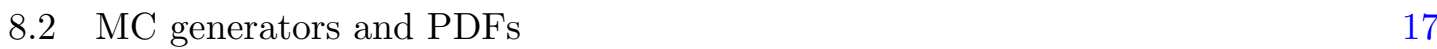

$\begin{array}{ll}\text { 8.3 Signal and background normalisation } & 18\end{array}$

$\begin{array}{lll}8.4 & \text { Detector correction and background parameterisation } & 18\end{array}$

$\begin{array}{ll}8.5 & \text { Uncertainty combination } \\ \end{array}$

9 Results 18

$\begin{array}{ll}10 \text { Conclusion } & 21\end{array}$

A Parameter dependence of the folding and background models 23

$\begin{array}{ll}\text { The ATLAS collaboration } & 29\end{array}$

\section{Introduction}

The top quark is the heaviest known fundamental particle, making the measurement of its production and decay kinematics a unique probe of physical processes beyond the Standard Model (SM). The top quark was first observed in 1995 at the Tevatron [1, 2] through its dominant production channel, top-quark pair $(t \bar{t})$ production via the flavour-conserving strong interaction. An alternative process produces single top quarks through the weak interaction, first observed at the Tevatron in 2009 [3, 4].

The $t$-channel exchange of a virtual $W$ boson is the dominant process contributing to single top-quark production (see figure 1). The production cross-section is calculated for 


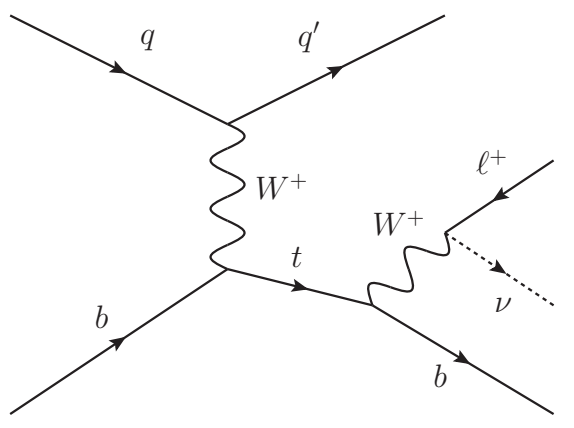

(a)

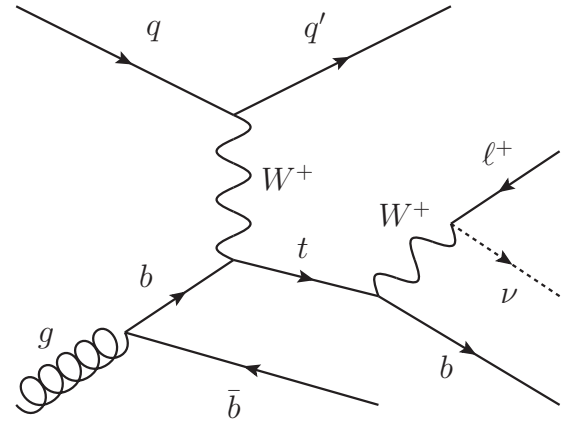

(b)

Figure 1. Representative Feynman diagrams for $t$-channel single top-quark production and decay. Here $q$ represents a $u$ or $\bar{d}$ quark, and $q^{\prime}$ represents a $d$ or $\bar{u}$ quark, respectively. The initial $b$-quark arises from (a) a sea $b$-quark in the $2 \rightarrow 2$ process, or (b) a gluon splitting into a $b \bar{b}$ pair in the $2 \rightarrow 3$ process.

proton-proton $(p p)$ collisions at $\sqrt{s}=7 \mathrm{TeV}$ using a next-to-leading-order (NLO) QCD prediction with resummed next-to-next-to-leading logarithmic (NNLL) accuracy, referred to as approximate next-to-next-to-leading order (NNLO). With a top-quark mass of $172.5 \mathrm{GeV}$ and MSTW2008NNLO [5] parton distribution function (PDF) sets, the cross-section for the $t$-channel process is calculated to be $64.6_{-1.7}^{+2.6} \mathrm{pb}$ [6]. The uncertainties correspond to the sum in quadrature of the error obtained from the MSTW PDF set at the $90 \%$ confidence level (C.L.) and the factorisation and renormalisation scale uncertainties.

New physics resulting in corrections to the $W t b$ vertex would affect $t$-channel single top-quark production and decay, and can be probed through processes which affect variables sensitive to the angular distributions of the final-state particles from the top-quark decay. Within the effective field theory framework, the Lagrangian can be expressed in full generality as $[7,8]$ :

$$
\mathcal{L}_{W t b}=-\frac{g}{\sqrt{2}} \bar{b} \gamma^{\mu}\left(V_{\mathrm{L}} P_{\mathrm{L}}+V_{\mathrm{R}} P_{\mathrm{R}}\right) t W_{\mu}^{-}-\frac{g}{\sqrt{2}} \bar{b} \frac{i \sigma^{\mu \nu} q_{\nu}}{m_{W}}\left(g_{\mathrm{L}} P_{\mathrm{L}}+g_{\mathrm{R}} P_{\mathrm{R}}\right) t W_{\mu}^{-}+\text {h.c. }
$$

where $g$ is the weak coupling constant, and $m_{W}$ and $q_{\nu}$ are the mass and the four-momentum of the $W$ boson, respectively. $P_{\mathrm{L}, \mathrm{R}} \equiv\left(1 \mp \gamma^{5}\right) / 2$ are the left- and right-handed projection operators and $\sigma^{\mu \nu}=i\left[\gamma^{\mu}, \gamma^{\nu}\right] / 2 . \quad V_{\mathrm{L}, \mathrm{R}}$ and $g_{\mathrm{L}, \mathrm{R}}$ are the complex left- and right-handed vector and tensor couplings, respectively. The couplings could also be expressed using the Wilson coefficients [9] of the relevant dimension-six operators, ${ }^{1}$ described in refs. [7, 10]. In the $\mathrm{SM}$ at tree level, $V_{\mathrm{L}}=V_{t b}$, which is an element in the Cabibbo-Kobayashi-Maskawa (CKM) matrix, while the anomalous couplings $V_{\mathrm{R}}=g_{\mathrm{L}, \mathrm{R}}=0$. Deviations from these values would provide hints of physics beyond the $\mathrm{SM}$, and complex values could imply that top-quark decay has a $\mathcal{C P}$-violating component [11].

\footnotetext{
${ }^{1}$ In general the couplings can depend on the momentum transfer $q^{2}$. Since this analysis is mainly sensitive to top-quark decays to an on-shell $W$ boson, where $q^{2}=m_{W}^{2}$, no $q^{2}$ dependence is considered.
} 
Indirect constraints on $V_{\mathrm{R}}, g_{\mathrm{L}}$, and $g_{\mathrm{R}}$ were obtained $[12,13]$ from precision measurements of $B$-meson decays. These result in very tight constraints on $V_{\mathrm{R}}$ and $g_{\mathrm{L}}$, but much looser constraints on $g_{\mathrm{R}}$. Calculations of the anomalous couplings in specific models predict a much larger contribution to $g_{\mathrm{R}}$ than to $V_{\mathrm{R}}$ or $g_{\mathrm{L}}$ [14]. However, within the models studied so far for tensor couplings, the magnitude of $g_{\mathrm{R}}$ is expected to be $<0.01[14,15]$. Measurements of the $W$ boson polarisation fractions [16-19] are sensitive to the magnitude of combinations of anomalous couplings. In order to obtain constraints on $\operatorname{Re}\left[g_{\mathrm{R}}\right]$ in those measurements, the anomalous couplings are taken to be purely real, $V_{\mathrm{R}}=g_{\mathrm{L}}=0$ is assumed and $V_{\mathrm{L}}$ is fixed to 1 . In this analysis, the anomalous couplings $V_{\mathrm{L}}$ and $g_{\mathrm{R}}$ are allowed to be complex, and the ratios $\operatorname{Re}\left[g_{\mathrm{R}} / V_{\mathrm{L}}\right]$ and $\operatorname{Im}\left[g_{\mathrm{R}} / V_{\mathrm{L}}\right]$ are measured.

This paper is organised as follows. Section 2 describes the coordinate system and parameterisation used in the measurement. Section 3 gives a short description of the ATLAS detector, then section 4 describes the simulated samples used to predict properties of the $t$-channel signal and the background processes, and the data samples used. Section 5 describes the definitions of the objects used to identify $t$-channel events, and the set of criteria applied to reduce the number of background events. The procedures for modelling background processes are described in section 6 , along with comparisons between the predictions and data. Section 7 describes the efficiency, resolution, and background models used to translate the distribution of true $t$-channel signal events to the distribution of reconstructed signal and background events, and how the parameters of the model are estimated. Section 8 quantifies the sources of uncertainty important in this measurement, section 9 presents the resulting central value and covariance matrix for the model parameters and the ratios $\operatorname{Re}\left[g_{\mathrm{R}} / V_{\mathrm{L}}\right]$ and $\operatorname{Im}\left[g_{\mathrm{R}} / V_{\mathrm{L}}\right]$, and the conclusions are given in section 10 .

\section{Measurement definition}

An event-specific coordinate system is defined for analysing the decay of the top quark in its rest frame using the directions of the jets, lepton, and $W$ boson in the final state, depicted in figure 2. The $\hat{z}$-axis is chosen along the direction of the $W$ boson boosted into the top-quark rest frame, $\hat{z} \equiv \hat{q}=\vec{q} /|\vec{q}|$. The angle $\theta^{*}$ between $\hat{q}$ and the momentum of the lepton in the $W$ boson rest frame, $\vec{p}_{\ell}$, is the same angle used to measure the $W$ boson helicity fractions in top-quark decays [17-19]. The spin of single top quarks produced in the $t$-channel, $\vec{s}_{t}$, is predicted to be polarised along the direction of the light quark $\left(q^{\prime}\right.$ in figure 1) in the top-quark rest frame [20], called the spectator-quark direction, $\hat{p}_{\mathrm{s}}$. If this jet defines the spin analysing direction, the degree of polarisation is shown in refs. [21, 22] to be $P \equiv \hat{p}_{\mathrm{s}} \cdot \vec{s}_{t} /\left|\vec{s}_{t}\right| \approx 0.9$ at $\sqrt{s}=7 \mathrm{TeV}$ for SM couplings. A three-dimensional coordinate system is defined from the $\hat{q}-\hat{p}_{\mathrm{s}}$ plane and the perpendicular direction, with $\hat{y}=\hat{p}_{\mathrm{s}} \times \hat{q}$ and $\hat{x}=\hat{y} \times \hat{q}$. In this coordinate system, the direction of the lepton $(\ell)$ in the $W$ boson rest frame $\hat{p}_{\ell}$ is specified by $\theta^{*}$ and the complementary azimuthal angle $\phi^{*}$.

These angles arise as a natural choice for measuring a normalised double differential distribution, in the helicity basis (top-quark rest frame), of the leptonic decay of the $W$ boson arising from the top quark. In this basis, the $t \rightarrow W b$ transition is determined by four helicity amplitudes, where the $W$ boson and $b$-quark are either both right-handed, both 


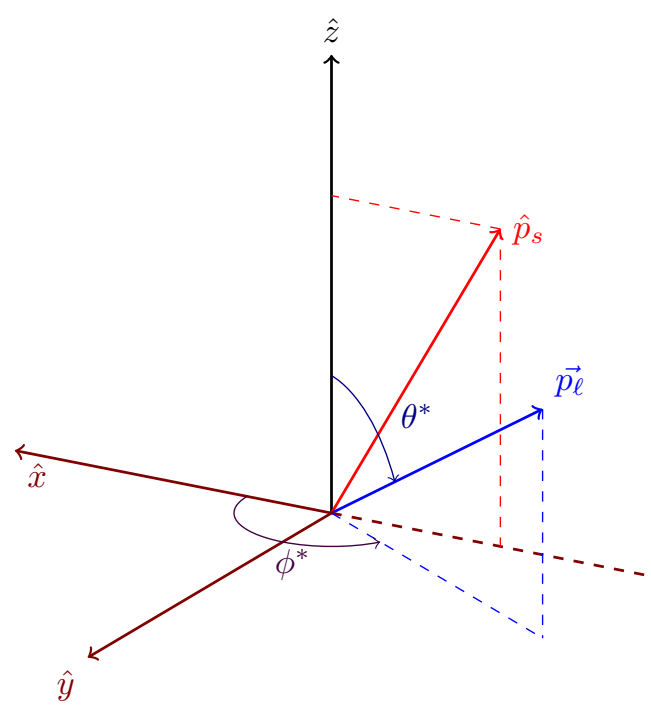

Figure 2. Definition of the coordinate system with $\hat{x}, \hat{y}$, and $\hat{z}$ defined as shown from the momentum directions of the $W$ boson, $\hat{q} \equiv \hat{z}$, and the spectator jet, $\hat{p}_{\mathrm{s}}$, in the top-quark rest frame. The angles $\theta^{*}$ and $\phi^{*}$ indicate the lepton direction $\hat{p}_{\ell}$ in this coordinate system.

left-handed, or the $W$ boson is longitudinal and the $b$-quark is either left- or right-handed. The resulting angular distribution of final-state leptons in $\theta^{*}$ and $\phi^{*}$ is expressed [23] as a series in spherical harmonics, $Y_{l}^{m}\left(\theta^{*}, \phi^{*}\right)$, parameterised by $\vec{\alpha}=\left(f_{1}, f_{1}^{+}, f_{0}^{+}, \delta_{+}, \delta_{-}\right)$and $P$ :

$$
\begin{aligned}
& \rho\left(\theta^{*}, \phi^{*} ; \vec{\alpha}, P\right) \equiv \frac{1}{N} \frac{\mathrm{d} N}{\mathrm{~d} \Omega^{*}}=\sum_{l=0}^{2} \sum_{m=-l}^{l} a_{l, m}(\vec{\alpha}, P) Y_{l}^{m}\left(\theta^{*}, \phi^{*}\right), \text { with } \\
& a_{0,0}=\frac{1}{\sqrt{4 \pi}}, \quad a_{1,0}=\frac{\sqrt{3}}{\sqrt{4 \pi}} f_{1}\left(f_{1}^{+}-\frac{1}{2}\right), \quad a_{2,0}=\frac{1}{\sqrt{20 \pi}}\left(\frac{3}{2} f_{1}-1\right), \\
& a_{1,1}=-a_{1,-1}^{*}=P \frac{\sqrt{3 \pi}}{16} \sqrt{f_{1}\left(1-f_{1}\right)}\left\{\sqrt{f_{1}^{+} f_{0}^{+}} e^{i \delta_{+}}+\sqrt{\left(1-f_{1}^{+}\right)\left(1-f_{0}^{+}\right)} e^{-i \delta_{-}}\right\}, \\
& a_{2,1}=-a_{2,-1}^{*}=P \frac{\sqrt{3 \pi}}{16 \sqrt{5}} \sqrt{f_{1}\left(1-f_{1}\right)}\left\{\sqrt{f_{1}^{+} f_{0}^{+}} e^{i \delta_{+}}-\sqrt{\left(1-f_{1}^{+}\right)\left(1-f_{0}^{+}\right)} e^{-i \delta_{-}}\right\}, \\
& a_{2,2}=a_{2,-2}=0 .
\end{aligned}
$$

The restriction to $l \leq 2$ in Equation (2.1) is caused by the limited spin states of the initial and final state fermions and the vector boson at the weak vertex.

The parameters $\vec{\alpha}$ describing this distribution can be expressed at tree level in terms of four linear combinations of the anomalous couplings. The fractions, $f_{1}, f_{1}^{+}$, and $f_{0}^{+}$, depend on the magnitude of these four combinations, while $\delta_{+}$and $\delta_{-}$depend on the phases between pairs of combinations. In addition there is a small correction due to the finite mass of the $b$-quark, $m_{b}$. Defining $x_{W}=m_{W} / m_{t}$ and $x_{b}=m_{b} / m_{t}$, where $m_{t}$ is the top-quark mass, the parameters are given by:

- $f_{1}$, the fraction of decays containing transversely polarised $W$ bosons,

$$
f_{1}=\frac{2\left(\left|x_{W} V_{\mathrm{L}}-g_{\mathrm{R}}\right|^{2}+\left|x_{W} V_{\mathrm{R}}-g_{\mathrm{L}}\right|^{2}\right)+\mathcal{O}\left(x_{b}\right)}{2\left(\left|x_{W} V_{\mathrm{L}}-g_{\mathrm{R}}\right|^{2}+\left|x_{W} V_{\mathrm{R}}-g_{\mathrm{L}}\right|^{2}\right)+\left|V_{\mathrm{L}}-x_{W} g_{\mathrm{R}}\right|^{2}+\left|V_{\mathrm{R}}-x_{W} g_{\mathrm{L}}\right|^{2}+\mathcal{O}\left(x_{b}\right)}
$$


- $f_{1}^{+}$, the fraction of transversely polarised $W$ boson decays that are right-handed,

$$
f_{1}^{+}=\frac{\left|x_{W} V_{\mathrm{R}}-g_{\mathrm{L}}\right|^{2}+\mathcal{O}\left(x_{b}\right)}{\left|x_{W} V_{\mathrm{L}}-g_{\mathrm{R}}\right|^{2}+\left|x_{W} V_{\mathrm{R}}-g_{\mathrm{L}}\right|^{2}+\mathcal{O}\left(x_{b}\right)}
$$

- $f_{0}^{+}$, in events with longitudinally polarised $W$ bosons, the fraction of $b$-quarks that are right-handed,

$$
f_{0}^{+}=\frac{\left|V_{\mathrm{R}}-x_{W} g_{\mathrm{L}}\right|^{2}+\mathcal{O}\left(x_{b}\right)}{\left|V_{\mathrm{R}}-x_{W} g_{\mathrm{L}}\right|^{2}+\left|V_{\mathrm{L}}-x_{W} g_{\mathrm{R}}\right|^{2}+\mathcal{O}\left(x_{b}\right)}
$$

- $\delta_{+}$, the phase between amplitudes for longitudinally polarised and transversely polarised $W$ bosons recoiling against right-handed $b$-quarks,

$$
\delta_{+}=\arg \left(\left(x_{W} V_{\mathrm{R}}-g_{\mathrm{L}}\right)\left(V_{\mathrm{R}}-x_{W} g_{\mathrm{L}}\right)^{*}+\mathcal{O}\left(x_{b}\right)\right)
$$

- $\delta_{-}$, the phase between amplitudes for longitudinally polarised and transversely polarised $W$ bosons recoiling against left-handed $b$-quarks,

$$
\delta_{-}=\arg \left(\left(x_{W} V_{\mathrm{L}}-g_{\mathrm{R}}\right)\left(V_{\mathrm{L}}-x_{W} g_{\mathrm{R}}\right)^{*}+\mathcal{O}\left(x_{b}\right)\right)
$$

- $P$, which is considered separately from $\vec{\alpha}$ because it depends on the production of the top quark, rather than the decay. There is no analytical expression for $P$ in terms of anomalous couplings, but a parameterisation is determined in ref. [24] by fitting simulated samples produced with the leading-order (LO) Protos [25] generator ${ }^{2}$ with different values for the various couplings.

Through these expressions, measurements of the parameterisation variables, $(\vec{\alpha}, P)$, can be used to set limits on the values of the couplings $V_{\mathrm{L}, \mathrm{R}}$ and $g_{\mathrm{L}, \mathrm{R}}$.

The parameters to which this analysis is most sensitive are the fraction $f_{1} \in[0,1]$ and the phase $\delta_{-} \in[-\pi, \pi]$. The parameter $f_{1}$ can be related to the $W$ boson helicity fractions via $f_{1}=F_{\mathrm{R}}+F_{\mathrm{L}}$, where $F_{\mathrm{R}}=f_{1} f_{1}^{+}$and $F_{\mathrm{L}}=f_{1}\left(1-f_{1}^{+}\right)$. Since $f_{1}^{+}$and $f_{0}^{+}$are small near the SM point, the term in Equation (2.1) that is proportional to $e^{i \delta_{+}}$is nearly zero. Thus $\delta_{+}$cannot be constrained and $f_{0}^{+}$cannot be separated from $P$. Constraints on $f_{1}^{+}$are currently better determined from limits on $F_{\mathrm{R}}[17,18]$. The variations that the parameters $f_{1}$ and $\delta_{-}$induce in the model are demonstrated in figure 3 for $t$-channel signal events generated with Protos. For regions of the parameter space $\left(f_{1}, \delta_{-}\right)$close to the SM, only these parameters can be significantly constrained. The dependence of $\delta_{-}$on $V_{\mathrm{R}}$ and $g_{\mathrm{L}}$ is suppressed by $x_{b}$, while both $f_{1}$ and $\delta_{-}$are dependent on $V_{\mathrm{L}}$ and $g_{\mathrm{R}}$ directly or through $x_{W}$. Thus, to simplify the analysis, only variations in $V_{\mathrm{L}}$ and $g_{\mathrm{R}}$ are considered, while $V_{\mathrm{R}}$ and $g_{\mathrm{L}}$ are assumed to be zero. With this assumption, the values of $f_{1}^{+}$and $f_{0}^{+}$are very small. The value of $P$ is also determined from the values of $V_{\mathrm{L}}$ and

\footnotetext{
${ }^{2}$ Protos (PROgram for TOp Simulations) is a generator for studying new physics processes involving the top quark. It has generators for single top-quark and top-quark pair production with anomalous $W t b$ couplings.
} 


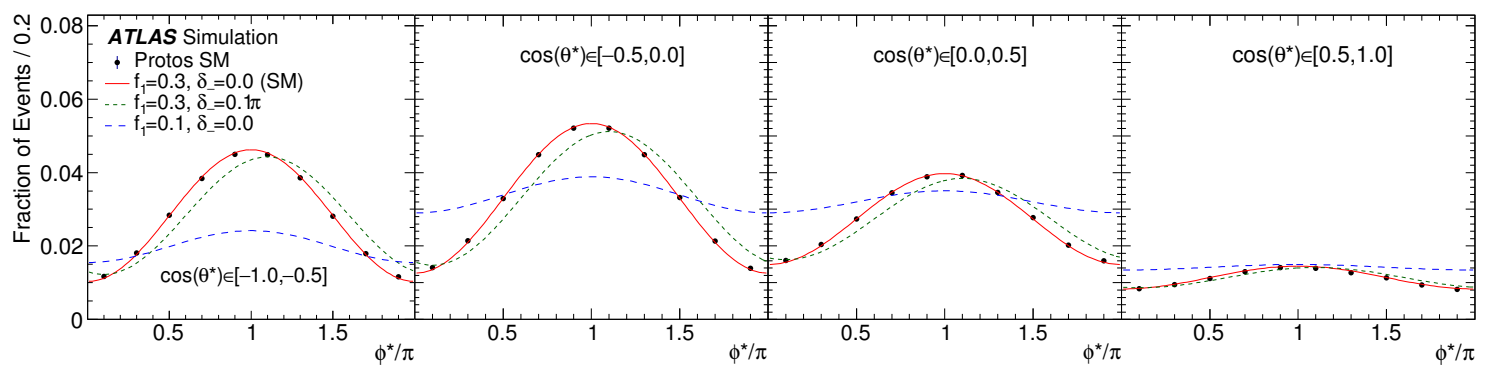

(a)

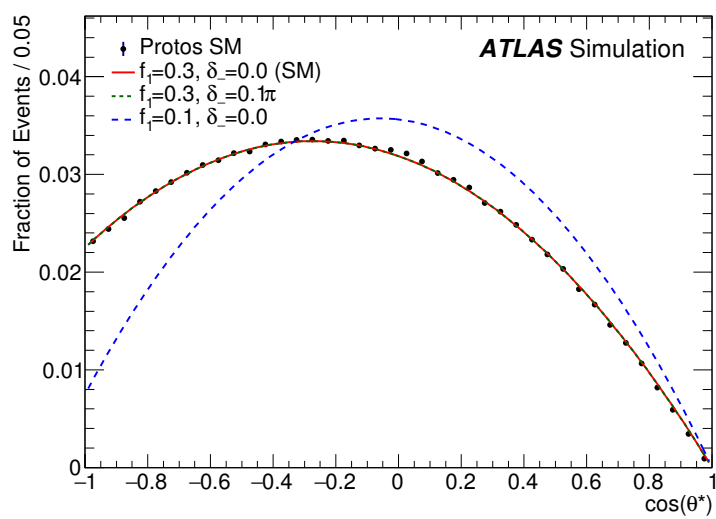

(b)

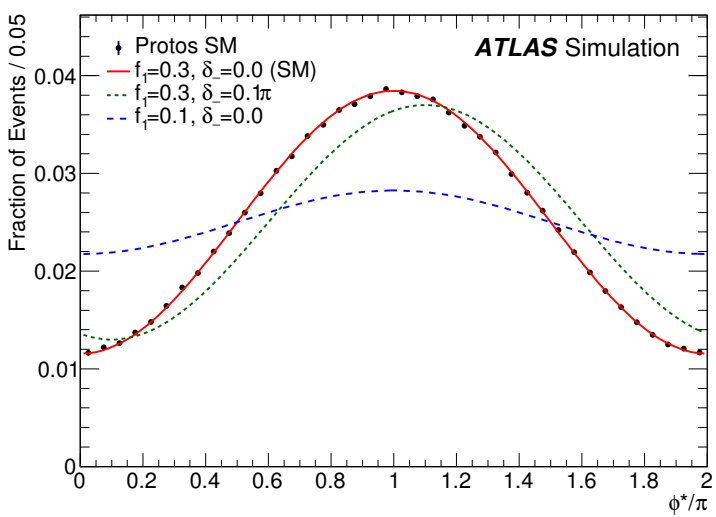

(c)

Figure 3. Projections into (a) $\phi^{*}$ in bins of $\cos \theta^{*}$, (b) $\cos \theta^{*}$, and (c) $\phi^{*}$ in Equation (2.1), for different values of $f_{1}$ and $\delta_{-}$. The black points represent the Protos $t$-channel signal generated with SM parameters, and the curves shown represent the signal model. For the three curves shown, the parameters $f_{1}$ and $\delta_{-}$are set to their values in the SM, $f_{1}=0.3, \delta_{-}=0$ (solid red), and to two sets of beyond-the-SM values, $f_{1}=0.1, \delta_{-}=0$ (dashed blue) and $f_{1}=0.3, \delta_{-}=0.1 \pi$ (dotted green).

$g_{\mathrm{R}}$. The highest-order dependence of $f_{1}$ and $\delta_{-}$on the couplings $V_{\mathrm{L}}$ and $g_{\mathrm{R}}$ appear as the ratio $\frac{g_{\mathrm{R}}}{V_{\mathrm{L}}}$, where the real and imaginary parts of this ratio are measured separately. This motivates quoting the results in both the parameter space $\left(f_{1}, \delta_{-}\right)$and the coupling space $\left(\operatorname{Re}\left[g_{\mathrm{R}} / V_{\mathrm{L}}\right], \operatorname{Im}\left[g_{\mathrm{R}} / V_{\mathrm{L}}\right]\right)$.

\section{The ATLAS detector}

The ATLAS detector [26] consists of a set of sub-detector systems, cylindrical in the central region and planar in the two end-cap regions, that cover almost the full solid angle around the interaction point. ${ }^{3}$ ATLAS is composed of an inner tracking detector (ID) close to the interaction point, surrounded by a superconducting solenoid providing a $2 \mathrm{~T}$ axial magnetic field, electromagnetic and hadronic calorimeters, and a muon spectrometer (MS).

\footnotetext{
${ }^{3}$ ATLAS uses a right-handed coordinate system with its origin at the nominal interaction point (IP) in the centre of the detector and the $z$-axis along the beam pipe. The $x$-axis points from the IP to the centre of the LHC ring, and the $y$-axis points upward. Cylindrical coordinates $(r, \phi)$ are used in the transverse plane, $\phi$ being the azimuthal angle around the beam pipe. The pseudorapidity is defined in terms of the polar angle $\theta$ as $\eta=-\ln \tan (\theta / 2)$.
} 
The ID consists of a silicon pixel detector, a silicon microstrip detector (SCT), providing tracking information within pseudorapidity $|\eta|<2.5$, and a straw-tube transition radiation tracker (TRT) that covers $|\eta|<2.0$. The central electromagnetic calorimeter is a lead and liquid-argon (LAr) sampling calorimeter with high granularity, and is divided into a barrel region that covers $|\eta|<1.475$ and end-cap regions that cover $1.375<|\eta|<$ 3.2. An iron/scintillator tile calorimeter provides hadronic energy measurements in the central pseudorapidity range. The forward regions are instrumented with LAr calorimeters covering $3.1<|\eta|<4.9$ for both the electromagnetic and hadronic energy measurements. The MS covers $|\eta|<2.7$ and consists of three large superconducting toroid magnets with eight coils each, a system of trigger chambers, and precision tracking chambers. The ATLAS detector has a three-level trigger system [27], used to select events to be recorded for offline analysis. The first-level trigger is hardware-based and uses a subset of the detector information to reduce the physical event rate from $40 \mathrm{MHz}$ to at most $75 \mathrm{kHz}$. The second and third levels are software-based and together reduce the event rate to about $300 \mathrm{~Hz}$.

\section{Data and simulated samples}

This analysis is performed using $p p$ collision data delivered by the LHC [28] in 2011 at $\sqrt{s}=7 \mathrm{TeV}$ and recorded by the ATLAS experiment. Stringent detector and data quality requirements are applied, resulting in a data sample corresponding to a total integrated luminosity of $4.59 \pm 0.08 \mathrm{fb}^{-1}$ [29]. The events are selected by un-prescaled single-lepton triggers $[27,30]$ that require a minimum transverse energy, $E_{\mathrm{T}}$, of $20 \mathrm{GeV}$ or $22 \mathrm{GeV}$ for electrons and a minimum transverse momentum, $p_{\mathrm{T}}$, of $18 \mathrm{GeV}$ for muons, depending on the data-taking conditions.

Samples of events generated using Monte Carlo (MC) simulations are produced for $t$ channel signal and the background processes, and are used to evaluate models of efficiency and resolution, and to estimate systematic uncertainties.

Samples of simulated $t$-channel single top-quark events are produced with the ACERMC multi-leg LO generator [31] (version 3.8) using the LO CTEQ6L1 [32] PDF sets. ACERMC incorporates both the $2 \rightarrow 2$ and $2 \rightarrow 3$ processes (see figure 1 ) and features an automated procedure to remove the overlap in phase space between them [33]. The factorisation and renormalisation scales are set to $\mu_{\mathrm{F}}=\mu_{\mathrm{R}}=172.5 \mathrm{GeV}$. Additional $t$-channel samples with different anomalous couplings are produced with PROTOS [25] (version 2.2) using the CTEQ6L1 PDF sets. Events are generated using PROTOs with the four-flavour scheme, ${ }^{4}$ incorporating only the $2 \rightarrow 3$ process, and anomalous couplings are enabled in both the production and the decay vertices, varying $\operatorname{Re}\left[V_{\mathrm{L}}\right]$ and $\operatorname{Im}\left[g_{\mathrm{R}}\right]$ simultaneously to keep the top-quark width invariant. The factorisation scale is set to $\mu_{\mathrm{F}}^{2}=-p_{W}^{2}$ for the light quark and $\mu_{\mathrm{F}}^{2}=p_{\bar{b}}^{2}+m_{b}^{2}$ for the gluon. These samples are used to evaluate $t$-channel generator modelling uncertainties as described in section 8.2. They are also used to derive folding models with non-SM values of the couplings, used for performing validation tests in MC simulation and measurements in real data, as described in section 7 . The

\footnotetext{
${ }^{4}$ In the four-flavour scheme, the PDFs only contain the quarks lighter than the $b$-quark.
} 
parton showering (PS), hadronisation and underlying-event (UE) modelling in these samples are simulated with Pyтнia [34] (version 6.426) using the Perugia 2011C set of tuned parameters (P2011C tune) [35] with the CTEQ6L1 [32] PDF sets.

Samples of $t \bar{t}$ events, $s$-channel single top-quark events, and associated production of a $W$ boson and top quark $(W t)$ are produced using the PowHEG-BOx NLO generator (version 1.0) coupled with the NLO CT10 [36] set of PDFs and interfaced with PYTHIA for showering and hadronisation using the P2011C tune with CTEQ6L1 PDF sets. Additional $t \bar{t}$ samples are produced with Protos (version 2.2) using the CTEQ6L1 PDF sets. These samples are used to evaluate the background model with non-SM values of the couplings. The PS, hadronisation and UE in these samples are simulated with PYTHIA (version 6.426) using the AUET2B tune [37] with the MRST LO** [38] PDF sets. All processes involving top quarks are produced assuming $m_{t}=172.5 \mathrm{GeV}$.

Vector-boson production in association with jets $(V+$ jets $)$ is simulated using the multileg LO ALPGEn generator [39] (version 2.13) using CTEQ6L1 PDF sets and interfaced to Herwig [40] (version 6.5.20) together with the Jimmy UE model [41] (version 4.31). The contributions of $W+$ light-jets and $W+$ heavy-jets $(W+b \bar{b}, W+c \bar{c}, W+c)$ are simulated separately. To remove overlaps between the $n$ and $n+1$ parton samples the MLM matching scheme [39] is used. Double counting between the inclusive $W+n$ parton samples and samples with associated heavy-quark pair production is removed using an overlap-removal algorithm based on parton-jet $\Delta R$ matching [42]. Diboson processes ( $W W, W Z$ and $Z Z$ ) are produced using the HERWIG generator with the MRST LO** PDF sets.

After the event-generation step, the samples are passed through the full simulation of the ATLAS detector [43] based on Geant4 [44]. Additional Protos samples are passed through the ATLFAST2 simulation $[43,45]$ of the ATLAS detector, which uses a fast simulation for the calorimeters and their response. They are then reconstructed using the same procedure as for data. The simulation includes the effect of multiple $p p$ collisions per bunch crossing (pile-up). The events are weighted such that the distribution of the average number of collisions per bunch crossing is the same as in data.

\section{Object definitions and event selection}

The definitions of objects used in the analysis, including electrons, muons, jets and $b$-tagged jets, and missing transverse momentum, as well as the basic event selection are chosen to be identical to those used for the $t$-channel cross-section measurements in ref. [46]. This analysis requires exactly one isolated charged light lepton (electron or muon) with transverse momentum $p_{\mathrm{T}}>25 \mathrm{GeV}$ and pseudorapidity $|\eta|<2.5$. Exactly one $b$-tagged jet with $|\eta|<2.5$ and exactly one untagged jet with $|\eta|<4.5$ are required, both with $p_{\mathrm{T}}>30 \mathrm{GeV}$. The second $b$-quark coming from gluon splitting as shown in figure $1(\mathrm{~b})$ can result in an additional $b$-tagged jet. This second $b$-tagged jet generally has a softer $p_{\mathrm{T}}$ spectrum and a broader $\eta$ distribution compared to the $b$-tagged jet produced in the top-quark decay. It is often not detected in the experiment and is thus not required in the event selection. To reject jets from pile-up collisions, the jet-vertex fraction $\epsilon_{\mathrm{JVF}}$, defined as the ratio of the $\sum p_{\mathrm{T}}$ of all tracks in the jet originating at the primary vertex to the $\sum p_{\mathrm{T}}$ 
of all tracks associated with the jet, is required to satisfy $\epsilon_{\mathrm{JVF}}>0.75$. The magnitude of the missing transverse momentum must be $E_{\mathrm{T}}^{\text {miss }}>30 \mathrm{GeV}$. Two additional multijet background rejection criteria are applied. The transverse mass of the lepton- $E_{\mathrm{T}}^{\text {miss }}$ system,

$$
m_{\mathrm{T}}\left(\ell, E_{\mathrm{T}}^{\mathrm{miss}}\right)=\sqrt{2 p_{\mathrm{T}}(\ell) \cdot E_{\mathrm{T}}^{\mathrm{miss}}\left[1-\cos \left(\Delta \phi\left(\ell, E_{\mathrm{T}}^{\mathrm{miss}}\right)\right)\right]},
$$

is required to be larger than $30 \mathrm{GeV}$. Also, a more stringent cut on the lepton $p_{\mathrm{T}}$ is applied to events in which the lepton and leading jet, $j_{1}$, are back-to-back,

$$
p_{\mathrm{T}}(\ell)>\max \left(25,40\left(1-\frac{\pi-\left|\Delta \phi\left(j_{1}, \ell\right)\right|}{\pi-1}\right)\right) \mathrm{GeV},
$$

where $\Delta \phi\left(j_{1}, \ell\right)$ is the difference in azimuthal angle between the lepton momentum and the leading jet.

The $W$ boson coming from the decay of the top quark can be reconstructed from the momenta of the lepton and the neutrino by using four-momentum conservation. The missing transverse momentum vector, with magnitude $E_{\mathrm{T}}^{\text {miss }}$, is used to represent the transverse component of the neutrino momentum and the longitudinal component $p_{\nu}^{z}$ is chosen such that the resulting $W$ boson is on its mass shell. A quadratic expression is found for $p_{\nu}^{z}$. If there are two possible real values, the value closer to zero is taken. If the solutions are complex, the assumption that the neutrino is the only contributor to the $E_{\mathrm{T}}^{\text {miss }}$ is not valid. Therefore, the $E_{\mathrm{T}}^{\text {miss }}$ is rescaled, preserving its direction, in order to have physical (real) solutions for $p_{\nu}^{z}$. If two solutions for $E_{\mathrm{T}}^{\text {miss }}$ are found, the one resulting in the smaller value of $\left|p_{\nu}^{z}\right|$ is taken. The top-quark momentum is then reconstructed from this $W$ boson momentum and the momentum of the $b$-tagged jet. Finally, the momenta of the $W$ boson and spectator jet are boosted into the top-quark rest frame to obtain $\vec{q}$ and $\vec{p}_{\mathrm{s}}$, used to generate the coordinate system in figure 2 , and the lepton is boosted into the $W$ boson rest frame to obtain $\vec{p}_{\ell}$.

In addition to this basic event selection, further discrimination between $t$-channel single top-quark events and background events is achieved by applying additional criteria:

- The pseudorapidity of the untagged jet must satisfy $|\eta|>2$, since the spectator jet tends to be forward in the $t$-channel signature.

- The sum of the $p_{\mathrm{T}}$ of all final-state objects, $H_{\mathrm{T}}$, must be larger than $210 \mathrm{GeV}$, since the $H_{\mathrm{T}}$ distributions of the backgrounds have their peaks just below this value.

- The mass of the top quark reconstructed from its decay products is required to be within $150-190 \mathrm{GeV}$, to reject background events from processes not involving top quarks.

- The distance in $\eta$ between the untagged jet and the $b$-tagged jet must be larger than 1 , to further reduce $t \bar{t}$ contributions.

These criteria and the basic event selection together define the signal region of the analysis.

Two control regions are defined, enhanced in each of the two dominant backgrounds, $t \bar{t}$ and $W+$ jets, to validate the modelling of the backgrounds by simulated events. In the 
control region where the $t \bar{t}$ background is enriched, events with exactly four jets, exactly one of which is $b$-tagged, passing the basic event selection together with the top-quark mass and $H_{\mathrm{T}}$ requirements are selected. In the control region where $W+$ jets backgrounds are enriched, events with exactly two jets passing the basic event selection and an inversion of the top-quark mass criterion are required.

\section{Background estimation and event yields}

The largest background contributions to single top-quark $t$-channel production in the signal region arise from $t \bar{t}$ production and from $W$ boson production in association with jets $(W+$ jets $)$. Events containing $t \bar{t}$ production are difficult to distinguish from single topquark events, as they also have real top quarks in the final state. Also, the production of a $W$ boson with two jets, where one is identified as containing a $b$-hadron, can mimic the $t$-channel final state. Multijet production via the strong interaction can enter the signal region as well, and data-driven techniques are required to accurately model it, as explained at the end of this section. Other backgrounds originate from $W t$ production and $s$-channel single top-quark, diboson $(W W, W Z$, and $Z Z)$ and $Z+$ jets production. Most of these background models are derived directly from MC simulation, but specialised procedures are implemented for $W+$ jets and multijet production.

The $t \bar{t}$ cross-section is calculated at NNLO in QCD including resummation of NNLL soft-gluon terms with Top $++2.0[47-53]$ and is found to be $177_{-11}^{+10} \mathrm{pb}$. The uncertainties due to the PDFs at $68 \%$ C.L. and $\alpha_{\mathrm{S}}$ are calculated following the PDF4LHC [54] prescription for the MSTW2008NNLO, CT10, and NNPDF2.3 [55] error PDF sets, and are added in quadrature to the scale uncertainty to yield a total uncertainty of $6 \%$. Single top-quark production cross-sections for $W t$ and $s$-channel production are calculated at approximate NNLO and are found to be $15.7 \pm 1.2 \mathrm{pb}$ [56] and $4.63_{-0.18}^{+0.20} \mathrm{pb}$ [57], respectively. The uncertainties correspond to the sum in quadrature of the uncertainty derived from the MSTW2008NNLO error PDF sets at 90\% C.L. and the scale uncertainties, yielding a final uncertainty of about $8 \%$ for $W t$ production and about $4 \%$ for $s$-channel production. The $Z+$ jets inclusive production cross-section is estimated with NNLO precision using the FEWZ program [58]. Diboson events $(W W, W Z$, and $Z Z)$ are normalised to the NLO cross-section prediction calculated with MCFM [59]. The uncertainty on the combined $Z+$ jets and diboson background is estimated to be $60 \%$ as in ref. [46].

For the production of a $W$ boson in association with jets, the shapes of the relevant kinematic distributions are predicted from the AlPGEN sample. They are initially normalised to make the inclusive $W$ cross-section correspond to the NNLO prediction using the FEWZ program [58], with the same scaling factor being applied to the AlPGEN prediction for the $W+b \bar{b}, W+c \bar{c}$, and $W+$ light-jets samples. The Alpgen prediction for the $W+c$ process is scaled by a factor that is obtained from a study based on NLO calculations using MCFM [59]. Data-driven techniques are then used to estimate the flavour composition and the overall normalisation as described in ref. [60], and an uncertainty of $18 \%$ $(15 \%)$ for electron (muon) selections is applied. 
Multijet background events pass the signal region selection if a jet is mis-identified as an isolated lepton or if the event has a non-prompt lepton from the decay of a hadron that appears to be isolated. Since it is neither possible to simulate a sufficient number of those events nor to calculate the rate precisely, dedicated techniques are used to model multijet events and to estimate their production rate, employing both collision data and simulated events. In the electron channel, mis-identified jets are the main source of multijet background events. This is modelled by the jet-lepton method [46], in which an electron-like jet is selected in PүтніA di-jet MC with special requirements and redefined as a lepton. In the muon channel, a data-driven matrix method [46, 61] based on the muon impact parameter significance is used. An uncertainty of $50 \%$ is assigned to the estimated yield based on comparisons of the rates obtained by using alternative methods [46], i.e. the matrix method in the electron channel and the jet-lepton method in the muon channel, and using an alternative variable, i.e. $m_{\mathrm{T}}\left(\ell, E_{\mathrm{T}}^{\mathrm{miss}}\right)$ instead of $E_{\mathrm{T}}^{\mathrm{miss}}$.

Table 1 provides the event yields for the electron and muon channels after the event selection. The predictions are normalised to $4.6 \mathrm{fb}^{-1}$ and their uncertainties are derived taking into account the statistical uncertainties of the simulated samples. For the multijet background, the uncertainties on the prediction are the statistical uncertainties of the data and simulated samples of the muon and electron channel, respectively. Figure 4 shows the angular distributions $\cos \theta^{*}$ and $\phi^{*}$ after the event selection for the electron and muon channels in data, compared to predictions using SM couplings. The uncertainties are derived taking into account the statistical uncertainties of the data samples for data and simulated samples on the prediction and the $50 \%$ systematic uncertainty on the normalisation of the multijet background. Since the number of events in $W$ +light-jets and multijet samples are low, statistical fluctuations and events with large generator weights affect the sample shapes. Therefore, shape templates from events selected without the $b$-tagging requirement are used for these two backgrounds to smooth the simulated models. In these events, the hardest jet in the event is chosen to take the place of the $b$-tagged-like jet for the purposes of the event selection and reconstruction. Overall, good agreement between the observed and expected distributions is found.

\section{Analysis method}

The model describing the $t$-channel signal in section 2 is connected to the angles measured in reconstructed events via an analytic folding procedure. Models of the selection efficiency and detector resolution are derived from simulated $t$-channel signal events, and a model of the reconstructed background is derived from the sum of the combined background processes described in section 4 . Since the values of the parameters $f_{1}$ and $\delta_{-}$depend on top-quark couplings, both the $t$-channel signal and the $t \bar{t}$ background are sensitive to their values. Only the angular distributions for the leptonic decay of the $W$ boson are measured, but the efficiency and resolution may also depend on other unmeasured distributions, such as those associated with the $\eta$ distribution of the top or spectator quark in the laboratory frame. The efficiency, resolution, and background models are constructed such that they account for any dependence on the values of $f_{1}$ and $\delta_{-}$, which is required to remove biases 


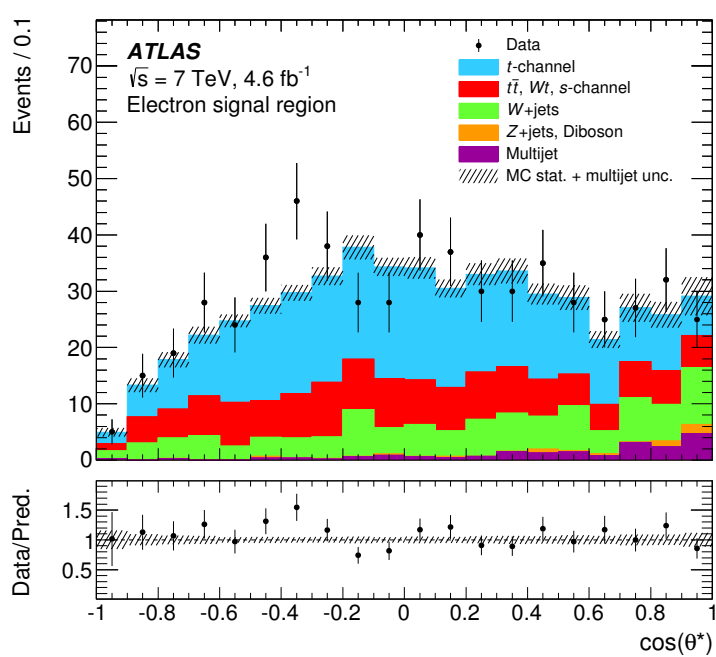

(a)

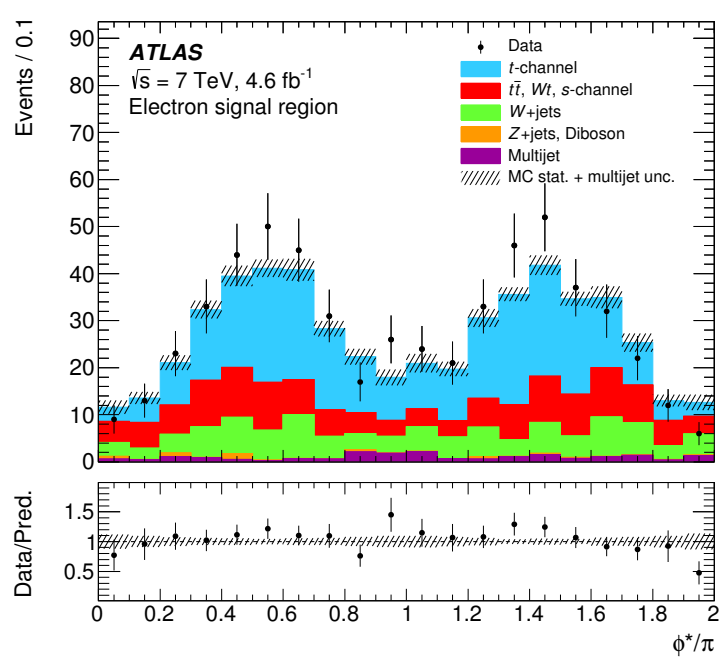

(c)

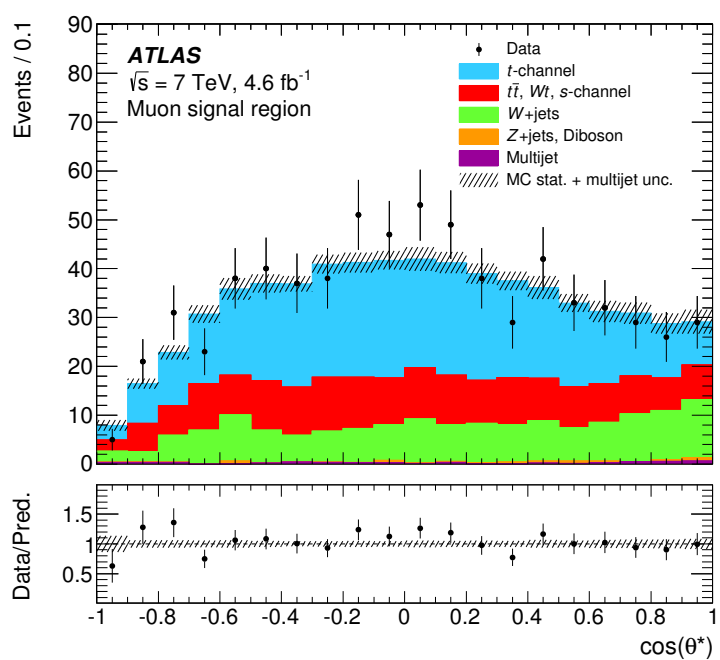

(b)

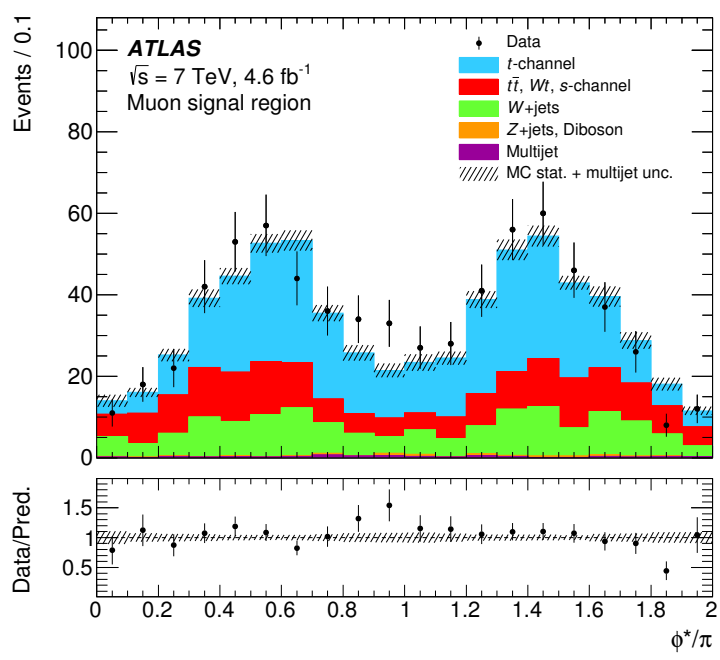

(d)

Figure 4. Angular distributions $\cos \theta^{*}$ (upper row) and $\phi^{*}$ (lower row) in the signal region for electrons (left) and muons (right), comparing data, shown as the black points with statistical uncertainties, to SM signal and background predictions. The uncertainties shown on the prediction take into account MC sample sizes and the $50 \%$ systematic uncertainty on the normalisation of the multijet background. The $W+$ jets backgrounds are normalised to the observed number of events in the control region, and the muon multijet backgrounds are predicted from observed data. All other samples are scaled to their theoretical predictions. The lower plots show the ratio of data to prediction in each bin. 


\begin{tabular}{|lr|r|}
\hline & Electrons & Muons \\
\hline$t$-channel & $274 \pm 2$ & $336 \pm 2$ \\
$s$-channel & $4 \pm 0$ & $5 \pm 0$ \\
$W t$-channel & $13 \pm 1$ & $16 \pm 1$ \\
$\bar{t}$ & $119 \pm 2$ & $147 \pm 3$ \\
$W+$ heavy-jets & $92 \pm 7$ & $128 \pm 8$ \\
$W+$ light-jets & $13 \pm 4$ & $18 \pm 3$ \\
$Z+$ light-jets & $4 \pm 1$ & $3 \pm 1$ \\
Diboson & $1 \pm 0$ & $2 \pm 0$ \\
Multijet & $20 \pm 7$ & $6 \pm 1$ \\
\hline Total expected & $538 \pm 11$ & $660 \pm 10$ \\
\hline Data & 576 & 691 \\
\hline
\end{tabular}

Table 1. Event yields for the electron and muon channels in the signal region. Individual predictions are rounded to integers while "Total expected" corresponds to the rounding of the sum of full precision individual predictions. The uncertainties shown are statistical only. Uncertainties of less than 0.5 events appear as zero.

dependent on the values of these parameters (see appendix A). A probability density is derived for all events in the signal region, as a function of the reconstructed angles $\cos \theta^{*}$ and $\phi^{*}$, and conditional on the parameters. This density is then used to construct a likelihood, from which $f_{1}$ and $\delta_{-}$are measured in data.

The signal model described by Equation (2.1) is a series in spherical harmonics, only containing terms up to a maximum value of $l, l_{\max }^{\text {sig }}=2$. Describing the efficiency and resolution models similarly allows the use of the orthogonality properties of spherical harmonics to construct analytically-folded distributions [23]. An efficiency function, given by

$$
\epsilon\left(\theta_{\mathrm{T}}^{*}, \phi_{\mathrm{T}}^{*} ; \vec{\alpha}, P\right)=\sum_{l^{\prime}, m^{\prime}}^{l_{\max }^{\mathrm{eff}}} e_{l^{\prime}, m^{\prime}}(\vec{\alpha}, P) Y_{l^{\prime}}^{m^{\prime}}\left(\theta_{\mathrm{T}}^{*}, \phi_{\mathrm{T}}^{*}\right),
$$

describes the probability that a $t$-channel signal event with the given true angles $\theta_{\mathrm{T}}^{*}$ and $\phi_{\mathrm{T}}^{*}$ will be reconstructed in the signal region. The series contains all allowed values of $l^{\prime}$ and $m^{\prime}$ up to a maximum spherical harmonic degree, $l_{\max }^{\text {eff }}$. The selected signal density, $\rho_{\mathrm{s}}$, is then defined as the product of the efficiency function and the signal model, $\rho$, normalised to the overall rate,

$$
\begin{aligned}
& \rho_{\mathrm{s}}\left(\theta_{\mathrm{T}}^{*}, \phi_{\mathrm{T}}^{*} ; \vec{\alpha}, P\right)=\frac{\epsilon\left(\theta_{\mathrm{T}}^{*}, \phi_{\mathrm{T}}^{*} ; \vec{\alpha}, P\right) \rho\left(\theta_{\mathrm{T}}^{*}, \phi_{\mathrm{T}}^{*} ; \vec{\alpha}, P\right)}{\int \epsilon\left(\theta_{\mathrm{T}}^{*}, \phi_{\mathrm{T}}^{*} ; \vec{\alpha}, P\right) \rho\left(\theta_{\mathrm{T}}^{*}, \phi_{\mathrm{T}}^{*} ; \vec{\alpha}, P\right) \mathrm{d} \Omega_{\mathrm{T}}^{*}}=\sum_{L, M} c_{L, M}(\vec{\alpha}, P) Y_{L}^{M}\left(\theta_{\mathrm{T}}^{*}, \phi_{\mathrm{T}}^{*}\right), \\
& \text { for } \quad c_{L, M}(\vec{\alpha}, P)=\frac{\sum_{l, l^{\prime}, m, m^{\prime}} e_{l^{\prime}, m^{\prime}}(\vec{\alpha}, P) a_{l, m}(\vec{\alpha}, P) G_{l, l^{\prime}, L}^{m, m^{\prime}, M}}{\sum_{l, m}(-1)^{m} e_{l,-m}(\vec{\alpha}, P) a_{l, m}(\vec{\alpha}, P)} .
\end{aligned}
$$

The Gaunt coefficients $G$ are defined in terms of Clebsch-Gordan coefficients $C_{l_{1} l_{2} L}^{m_{1} m_{2} M}$, 
given by,

$$
G_{l, l^{\prime}, L}^{m, m^{\prime}, M}=\sqrt{\frac{(2 l+1)\left(2 l^{\prime}+1\right)}{4 \pi(2 L+1)}} C_{l, l^{\prime}, L}^{0,0,0} C_{l, l^{\prime}, L}^{m, m^{\prime}, M} .
$$

The efficiency function is determined from a likelihood fit to simulated $t$-channel events. A value is chosen for $l_{\max }^{\text {eff }}$ by comparing the associated values of the corrected Akaike Information Criteria, AICc [62, 63], a likelihood-ratio test with an additional penalty term which increases with the number of parameters. From this test, $l_{\max }^{\mathrm{eff}}=6$ is chosen. The deviation in the results from the selection of efficiency models with $l_{\max }^{\text {eff }}$ varied by \pm 2 are included as systematic uncertainties. Other criteria, such as the Schwarz Criteria [64], select values of $l_{\max }^{\mathrm{eff}}$ within the range chosen for this uncertainty.

When reconstructing events in the signal region, the finite resolution of the detector results in a migration from the true angles to measured angles $\theta^{*}$ and $\phi^{*}$. This migration is modelled by the resolution function,

$$
\mathcal{R}\left(\theta^{*}, \phi^{*} \mid \theta_{\mathrm{T}}^{*}, \phi_{\mathrm{T}}^{*} ; \vec{\alpha}, P\right)=\sum_{\lambda, \mu}^{l_{\text {max }}^{\text {reco }}} \sum_{L^{\prime}, M^{\prime}}^{l_{\text {max }}^{\text {true }}} r_{\lambda, \mu, L^{\prime}, M^{\prime}}(\vec{\alpha}, P) Y_{\lambda}^{\mu}\left(\theta^{*}, \phi^{*}\right) Y_{L^{\prime}}^{M^{\prime}}\left(\theta_{\mathrm{T}}^{*}, \phi_{\mathrm{T}}^{*}\right) .
$$

This series contains all terms with allowed values of $\lambda, \mu, L^{\prime}$, and $M^{\prime}$ up to the maximum degree parameters $l_{\max }^{\text {true }}$, associated with the dependence on $\theta_{\mathrm{T}}^{*}$ and $\phi_{\mathrm{T}}^{*}$, and $l_{\max }^{\text {reco }}$, associated with the dependence on $\theta^{*}$ and $\phi^{*}$. The reconstructed signal density, $\rho_{\mathrm{r}}$, is defined as the convolution of this function with the selected signal density,

$$
\begin{aligned}
\rho_{\mathrm{r}}\left(\theta^{*}, \phi^{*} ; \vec{\alpha}, P\right) & =\int \mathcal{R}\left(\theta^{*}, \phi^{*} \mid \theta_{\mathrm{T}}^{*}, \phi_{\mathrm{T}}^{*} ; \vec{\alpha}, P\right) \rho_{s}\left(\theta_{\mathrm{T}}^{*}, \phi_{\mathrm{T}}^{*} ; \vec{\alpha}, P\right) \mathrm{d} \Omega_{\mathrm{T}}^{*} \\
& =\sum_{\lambda, \mu} d_{\lambda, \mu}(\vec{\alpha}, P) Y_{\lambda}^{\mu}\left(\theta^{*}, \phi^{*}\right), \\
\text { for } \quad d_{\lambda, \mu}(\vec{\alpha}, P) & =\sum_{L, M}(-1)^{M} c_{L, M}(\vec{\alpha}, P) r_{\lambda, \mu, L,-M}(\vec{\alpha}, P) .
\end{aligned}
$$

The resolution function is determined using a spherical Fourier technique [65]. In principle, describing a very narrow resolution function could require large values of $l_{\max }^{\text {true }}$ and $l_{\max }^{\text {reco }}$. In practice, the mathematics of angular-momentum addition guarantee that there are no terms in Equation (7.2) with $L>l_{\max }^{\text {sig }}+l_{\max }^{\text {eff }}$. Thus, $l_{\max }^{\text {true }}=l_{\max }^{\text {sig }}+l_{\max }^{\text {eff }}$. The parameter $l_{\max }^{\text {reco }}$ is determined using the mean integrated squared error, MISE $=\int(\rho(x)-\hat{\rho}(x))^{2} \mathrm{~d} x$, where $\rho(x)$ is the true probability density and $\hat{\rho}(x)$ is a distribution estimating that density. Calculating the MISE with $\rho_{\mathrm{r}}$ yields a broad minimum for $l_{\max }^{\text {reco }}>4$, and for this analysis $l_{\max }^{\text {reco }}=6$ is chosen. The deviation in the results from the selection of resolution models with $l_{\max }^{\text {reco }}$ varied by \pm 2 are included as systematic uncertainties.

This density of reconstructed $t$-channel signal events is a series in spherical harmonics, with coefficients which are functions of the physics parameters describing the production $(P)$ and decay $(\vec{\alpha})$ of the top quark. A background model,

$$
\beta\left(\theta^{*}, \phi^{*} ; \vec{\alpha}, P\right)=\sum_{\lambda, \mu}^{l_{\max }^{\mathrm{bkg}}} b_{\lambda, \mu}(\vec{\alpha}, P) Y_{\lambda}^{\mu}\left(\theta^{*}, \phi^{*}\right)
$$


is derived which is also a series in spherical harmonics, and is added to the reconstructed signal density via the signal fraction, $f_{\mathrm{s}}$. This background function is determined from a likelihood fit to all simulated background events in the signal region, and contains all terms with allowed values of $\lambda$ and $\mu$ up to the maximum degree parameter $l_{\max }^{\mathrm{bkg}}$. This parameter is determined like the equivalent parameter in the efficiency function, using the AICc. The variations in the results from the selection of background models with $l_{\max }^{\mathrm{bkg}} \pm 2$ are included as systematic uncertainties.

The probability density of all events in the signal region, $\rho_{\mathrm{t}}$, is the sum of the reconstructed signal and background densities with signal fraction $f_{\mathrm{s}}$,

$$
\begin{aligned}
\rho_{\mathrm{t}}\left(\theta^{*}, \phi^{*} ; \vec{\alpha}, P, f_{\mathrm{s}}\right) & =\sum_{\lambda, \mu}^{\max \left(l_{\mathrm{max}}^{\mathrm{reco}}, l_{\max }^{\mathrm{bkg}}\right)} \mathcal{A}_{\lambda, \mu}(\vec{\alpha}, P) Y_{\lambda}^{\mu}\left(\theta^{*}, \phi^{*}\right) \text { for } \\
\mathcal{A}_{\lambda, \mu}\left(\vec{\alpha}, P, f_{\mathrm{s}}\right) & =f_{s} d_{\lambda, \mu}(\vec{\alpha}, P)+\left(1-f_{s}\right) b_{\lambda, \mu} \\
& =f_{s} \frac{\sum_{l, l^{\prime}, L, m, m^{\prime}, M}(-1)^{M} e_{l^{\prime}, m^{\prime}} a_{l, m}(\vec{\alpha}, P) G_{l, l^{\prime}, L}^{m, m^{\prime}, M} r_{\lambda, \mu, L,-M}}{\sum_{l, m}(-1)^{m} e_{l,-m} a_{l, m}(\vec{\alpha}, P)}+\left(1-f_{s}\right) b_{\lambda, \mu} .
\end{aligned}
$$

Figure 5 shows the data summed over the electron and muon channels for the signal region. Three representative models are also shown: one for the SM expectations, one that is near the expected $95 \%$ C.L. sensitivity in $f_{1}$, and one near the expected sensitivity in $\delta_{-}$. These values for $f_{1}$ and $\delta_{-}$are the same as those used in figure 3 , but the curves now include the effects of efficiency, resolution and background. The main differences between the two figures are due to the isolation requirements placed on the leptons. For $\cos \theta^{*}=-1$, the lepton overlaps the $b$-tagged jet, while for $\phi^{*}=\pi$, the lepton may overlap the spectator jet. The region where the efficiency is less than $0.05 \%\left(\cos \theta^{*}<-0.95\right.$, and $-0.95<\cos \theta^{*}<-0.85$ for $\pi-0.1<\phi^{*}<\pi+0.1$ ), is excluded from further analysis. For other regions, the efficiency varies, as a function of $\phi^{*}$ and $\cos \theta^{*}$, from $0.1 \%$ up to $1.7 \%$ for larger $\cos \theta^{*}$ and away from the plane containing the spectator jet. As the features of the angular distributions are broad, the effects of resolution are small. The main effect is to smooth out some of the effects of the acceptance and to increase the number of events at large $\cos \theta^{*}$ where the SM contribution is small. As seen in figure 4 , the background is also somewhat larger for large $\cos \theta^{*}$.

An unbinned likelihood function is constructed from the probability density in Equation (7.6) and from a set of events $\mathcal{D}=\left\{\theta_{i}^{*}, \phi_{i}^{*}, w_{i}\right\}, i=1,2, \ldots N$,

$$
\mathcal{L}(\vec{\alpha})=\prod_{i=1}^{N} \exp \left(w_{i} \rho_{\mathrm{t}}\left(\theta_{i}^{*}, \phi_{i}^{*} \mid \vec{\alpha}, P, f_{\mathrm{s}}\right)\right),
$$

where $\theta_{i}^{*}$ and $\phi_{i}^{*}$ can be taken either from data or MC simulation, and the event weights $w_{i}$ are 1 for measured data. The likelihood is evaluated on a grid with spacing 0.01 in $f_{1}$ and $0.01 \pi$ in $\delta_{-}$. The efficiency and resolution models at each point are derived from РRотоs $t$-channel simulated events, and the background model uses Protos $t \bar{t}$ simulated events. 


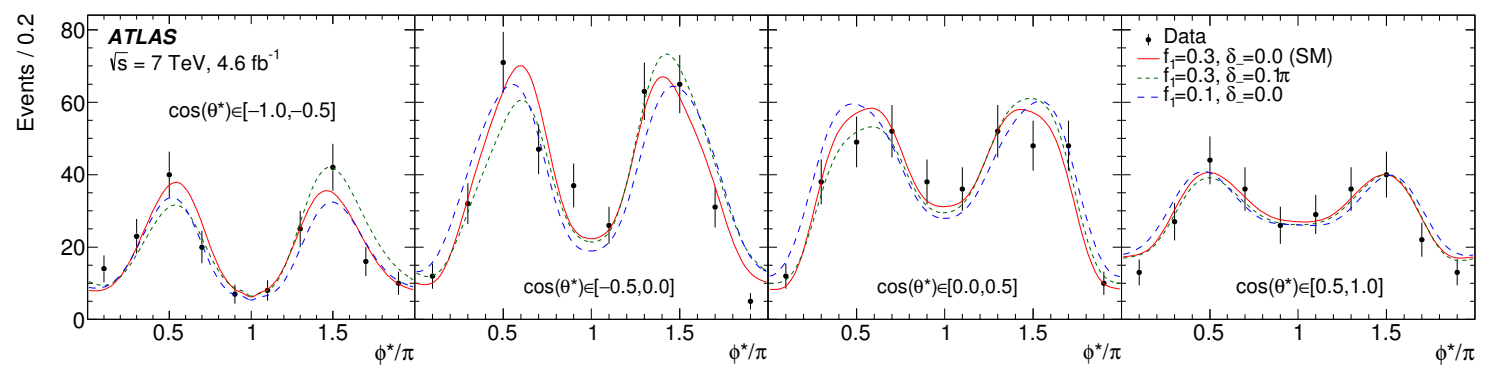

(a)

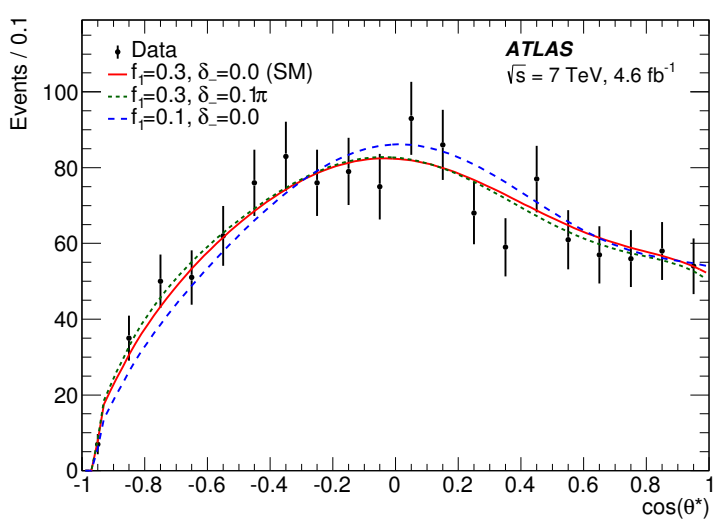

(b)

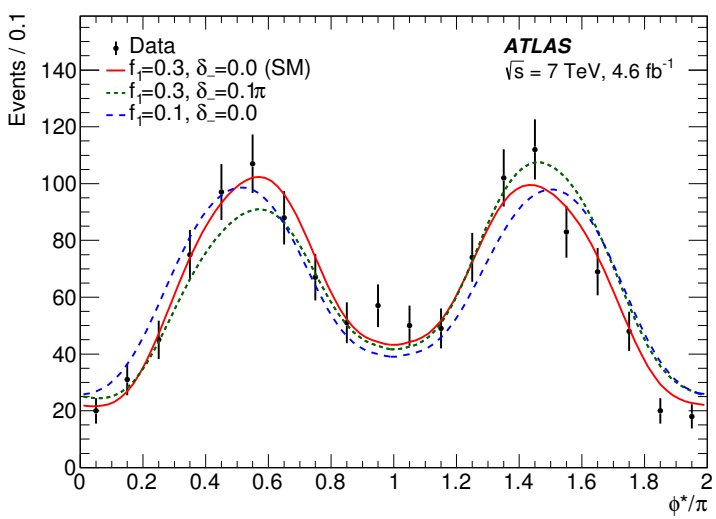

(c)

Figure 5. Projections into (a) $\phi^{*}$ in bins of $\cos \theta^{*}$, (b) $\cos \theta^{*}$, and (c) $\phi^{*}$ of the function described in Equation (7.6), for different values of $f_{1}$ and $\delta_{-}$. The black points shown are for the selected data events with statistical uncertainties. The curves shown represent the model at the SM point $f_{1}=0.3, \delta_{-}=0$ (solid red), and two sets of beyond-the-SM values, $f_{1}=0.1, \delta_{-}=0$ (dashed blue) and $f_{1}=0.3, \delta_{-}=0.1 \pi$ (dotted green).

Interpreting these parameters as varying the coupling ratio $g_{\mathrm{R}} / V_{\mathrm{L}}$, the polarisation $P$ is varied simultaneously according to the parameterisation in ref. [24].

A central value is measured at the maximum value of $\mathcal{L}$ on this grid, determined from a Gaussian fit to the points at which $\mathcal{L}$ is evaluated. The $68 \%$ and $95 \%$ confidence limits are defined by the region where the likelihood ratio, $\mathcal{L} / \mathcal{L}_{\max }$, is larger than the value that would yield the same likelihood for a two-dimensional Gaussian distribution. They are refined to increase the accessible precision by interpolating between points on either side of the contours determined from these evaluated points. The statistical uncertainty is estimated from the symmetrised $68 \%$ C.L. interval of each parameter.

This model is checked for closure both with MC samples, and by defining toy-MC samples based on Equation (7.6) with the same number of events as in data. These samples were generated at various points in $\left(f_{1}, \delta_{-}\right)$space. In all cases they are found to reproduce the expected values of $f_{1}$ and $\delta_{-}$. These toy-MC samples were also used to derive pull distributions for $f_{1}$ and $\delta_{-}$to check the statistical uncertainties returned by the fit. 


\section{Sources of systematic uncertainty}

Systematic uncertainties are evaluated in the full $\left(f_{1}, \delta_{-}\right)$parameter space. Efficiency, resolution, and background models are determined from MC samples with a parameter varied by its uncertainty, or a subset where appropriate. A likelihood is constructed from the resulting model, using events generated with nominal values of the varied parameters. The difference between the central values estimated at the nominal value of a parameter and at the value varied by its uncertainty, or half the difference between central values estimated with the parameter varied up and down by its uncertainty, is used to construct a covariance matrix for each source of systematic uncertainty. The total covariance matrix for the systematic uncertainties and its correlation matrix are found from the sum of the covariance matrices determined for individual uncertainties.

\subsection{Object modelling}

The uncertainties in the reconstruction of jets, leptons, and $E_{\mathrm{T}}^{\mathrm{miss}}$ are propagated to the analysis, following the same procedures as described in ref. [46]. The main source of uncertainty from these physics objects is the jet energy scale (JES) [66], which is the largest systematic uncertainty on the measurement of $f_{1}$. To estimate the impact of the JES uncertainty on the result, the jet energy is scaled up and down by its uncertainty [66], which ranges from $2.5 \%$ in the central region with high- $p_{\mathrm{T}}$ jets to $14 \%$ in the far forward region with low- $p_{\mathrm{T}}$ jets. Uncertainties are also estimated for jet energy resolution and reconstruction efficiency; the impact of varying the jet-vertex fraction requirement; $E_{\mathrm{T}}^{\text {miss }}$ reconstruction and the effect of pile-up collisions on the $E_{\mathrm{T}}^{\text {miss }} ; b$-tagging efficiency and mistagging rate; lepton trigger, identification, and reconstruction efficiencies; and lepton momentum, energy scale, and resolution.

\subsection{MC generators and PDFs}

Multiple MC event generators are used to model the $t$-channel and $t \bar{t}$ processes in this analysis, and the differences between these generators are included as systematic uncertainties. Comparing the ACERMC generator used for $t$-channel events and the PowHEG-BOX generator used for $t \bar{t}$ events to the PROTOs generator used for both the $t$-channel single top-quark events and $t \bar{t}$ events yields the largest uncertainty in $\delta_{-}$. Additional $t$-channel comparisons are performed between the ACERMC and POWHEG-BOX generators and between ACERMC events with showering by Pythia and HeRwig. The renormalisation and factorisation scales in the PowHEG-BOx $t$-channel sample are also varied independently by factors of 0.5 and 2.0. Additional $t \bar{t}$ comparisons are performed between the Powheg-BOX generator and the MC@NLO [67] generator with showering by HERwiG, and between Powheg-Box events with showering by Pythia and Herwig. The variations between PDF sets and within individual sets are used to estimate a systematic uncertainty following the PDF4LHC prescription. 


\subsection{Signal and background normalisation}

Cross-sections for background processes given in section 6 are varied up and down by their uncertainties. The multijet normalisation is varied by $50 \%$ as discussed in section 6 , and the remaining backgrounds are varied simultaneously to produce a conservative estimate. The impact of object modelling and other uncertainties on the $W+$ jets background normalisation are considered in parallel with the variations made in $t$-channel and $t \bar{t}$ samples. A separate shape uncertainty is assigned to the $W+$ jets samples by varying the matching and factorisation scales in the ALPGEN generator. The integrated luminosity is varied up and down by its uncertainty, $\pm 1.8 \%$, derived as detailed in ref. [29].

\subsection{Detector correction and background parameterisation}

An uncertainty due to the limited size of the MC samples used to estimate the efficiency and resolution models is estimated from the statistical uncertainties derived from a measurement of $f_{1}$ and $\delta_{-}$in the $t$-channel signal MC sample alone. The background statistical uncertainty is estimated by varying the background model according to the eigenvectors of its covariance matrix. The background statistical uncertainty dominates the total "MC statistics" uncertainty listed in table 2. Its significance reflects the small size of some background samples in the signal region, and the resulting disparate values of the sample weights. The effect of varying the cutoff degree $l_{\max }$ in the determination of these models is also estimated, and is found to be small compared to MC statistical uncertainty.

\subsection{Uncertainty combination}

Table 2 shows the contribution of each source of uncertainty to the measurement of the parameters $f_{1}$ and $\delta_{-}$and their correlation, $\rho\left(f_{1}, \delta_{-}\right)$. The total systematic uncertainty and correlation is obtained from the sum of the covariance matrices determined for each source. It is combined with the covariance matrix of the statistical uncertainty via the following method. At each point $\vec{\alpha}_{i}$ in the $\left(f_{1}, \delta_{-}\right)$space, a multivariate normal distribution $\mathcal{N}_{i}$ is constructed with the covariance matrix representing the systematic uncertainties, $\boldsymbol{\Sigma}^{\text {syst }}$, and the mean, $\vec{\alpha}_{i}$. The resulting distribution is evaluated at a point $\vec{\alpha}_{j}$ and multiplied by the likelihood at this point, $\mathcal{L}_{j}^{\text {stat }}$. The maximum modified likelihood value, over all possible points $\vec{\alpha}_{j}$, is kept, and the resulting broadened likelihood distribution $\mathcal{L}_{i}^{\text {stat+syst }}$ is used to represent the measurement with both statistical and systematic variation incorporated:

$$
\mathcal{L}_{i}^{\text {stat+syst }}=\max _{j}\left\{\mathcal{N}_{i}\left(\overrightarrow{\alpha_{j}} ; \vec{\alpha}_{i}, \Sigma^{\text {syst }}\right) \cdot \mathcal{L}_{j}^{\text {stat }}\right\} .
$$

The resulting confidence limits are taken as the full uncertainty in the measurement.

\section{Results}

The result for $\left(f_{1}, \delta_{-}\right)$and the coupling ratios $\left(\operatorname{Re}\left[g_{\mathrm{R}} / V_{\mathrm{L}}\right], \operatorname{Im}\left[g_{\mathrm{R}} / V_{\mathrm{L}}\right]\right)$ is shown in figure 6 . The $68 \%$ contour represents the total uncertainty on the measurement.

The parameters $f_{1}$ and $\delta_{-}$and their uncertainties are measured to be

$$
\begin{aligned}
f_{1} & =0.37 \pm 0.05 \text { (stat.) } \pm 0.05 \text { (syst.), } \\
\delta_{-} & =-0.014 \pi \pm 0.023 \pi \text { (stat.) } \pm 0.028 \pi \text { (syst.). }
\end{aligned}
$$




\begin{tabular}{|l|r|r|r|}
\hline Source & $\sigma\left(f_{1}\right)$ & $\sigma\left(\delta_{-}\right) / \pi$ & $\rho\left(f_{1}, \delta_{-}\right)$ \\
\hline Data statistics & 0.05 & 0.023 & 0.01 \\
\hline Jets & 0.03 & 0.015 & 0.39 \\
$b$-tagging & $<0.01$ & $<0.001$ & -0.70 \\
Leptons & 0.02 & 0.007 & 0.39 \\
$E_{\mathrm{T}}^{\text {miss }}$ & 0.01 & 0.004 & -0.27 \\
\hline Generator & 0.02 & 0.017 & 0.40 \\
Parton shower & 0.02 & 0.001 & 0.98 \\
PDF variations & 0.01 & 0.009 & 0.23 \\
\hline Cross-sections & $<0.01$ & $<0.001$ & 1.00 \\
$W+$ jets shape & $<0.01$ & 0.001 & -0.59 \\
Multijet normalisation & $<0.01$ & 0.002 & -1.00 \\
Luminosity & $<0.01$ & $<0.001$ & -1.00 \\
\hline Model $l_{\text {max variation }}$ & 0.01 & 0.001 & -0.70 \\
MC statistics & 0.02 & 0.011 & 0.14 \\
\hline Combined systematic & 0.05 & 0.028 & 0.27 \\
\hline Total & 0.07 & 0.036 & 0.15 \\
\hline
\end{tabular}

Table 2. Sources of systematic uncertainty on the measurement of $f_{1}$ and $\delta_{-}$using ACERMC $t$ channel single top-quark simulated events and backgrounds estimated from both MC simulation and data, including Powheg-Box $t \bar{t}$ simulation. Individual sources are evaluated separately for shifts up and down, and symmetrised uncertainties $\sigma\left(f_{1}\right), \sigma\left(\delta_{-}\right)$and correlation coefficients $\rho\left(f_{1}, \delta_{-}\right)$ are given.

The correlation in the measurement of these parameters is $\rho\left(f_{1}, \delta_{-}\right)=0.15$. The results are compatible with the SM expectations at LO, derived from expressions in refs. [11, 68] with $m_{t}=172.5 \mathrm{GeV}, m_{W}=80.399 \mathrm{GeV}$, and $m_{b}=4.95 \mathrm{GeV}: f_{1}=0.304$ and $\delta_{-}=0$.

The dependence of the parameters $f_{1}$ and $\delta_{-}$on the top-quark mass is evaluated using $t$-channel and $t \bar{t}$ simulation samples with a range of different top-quark masses. A linear dependence is found, resulting from changes in acceptance at different masses, with a slope of $-0.019 \mathrm{GeV}^{-1}$ for $f_{1}$ and a negligible slope for $\delta_{-}$. The uncertainty due to the topquark mass dependence is not included in the total systematic uncertainty since it has no significant impact on the results.

The propagation of the uncertainties to the $\left(\operatorname{Re}\left[g_{\mathrm{R}} / V_{\mathrm{L}}\right], \operatorname{Im}\left[g_{\mathrm{R}} / V_{\mathrm{L}}\right]\right)$ space gives

$$
\begin{aligned}
& \operatorname{Re}\left[\frac{g_{\mathrm{R}}}{V_{\mathrm{L}}}\right]=-0.13 \pm 0.07 \text { (stat.) } \pm 0.10 \text { (syst.), } \\
& \operatorname{Im}\left[\frac{g_{\mathrm{R}}}{V_{\mathrm{L}}}\right]=0.03 \pm 0.06 \text { (stat.) } \pm 0.07 \text { (syst.). }
\end{aligned}
$$

The correlation in the measurement of these coupling ratios is $\rho\left(\operatorname{Re}\left[g_{\mathrm{R}} / V_{\mathrm{L}}\right], \operatorname{Im}\left[g_{\mathrm{R}} / V_{\mathrm{L}}\right]\right)=0.11$. The effect on the propagation due to the current 


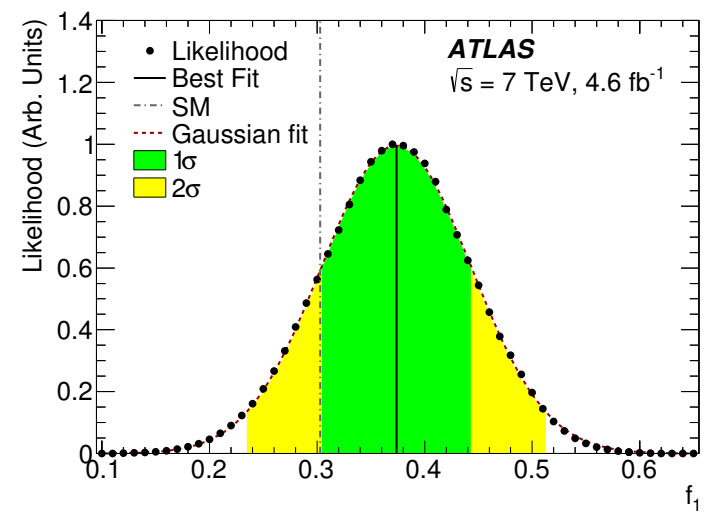

(a)

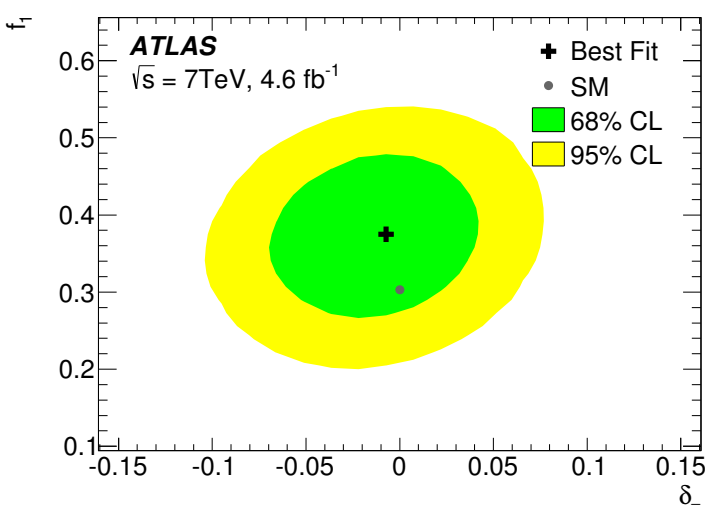

(c)

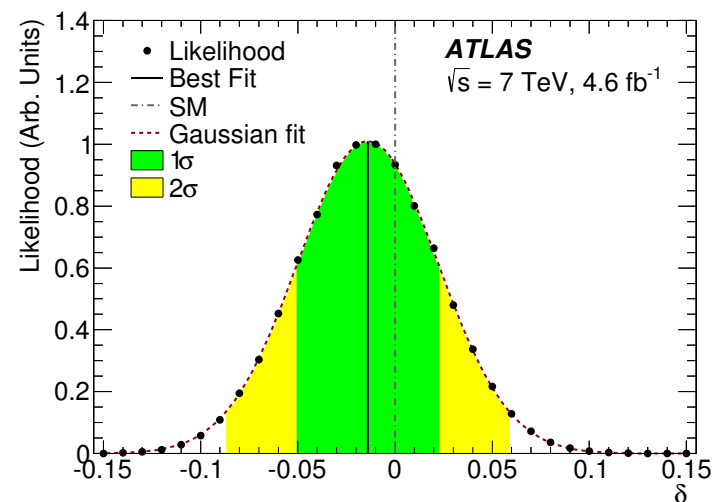

(b)

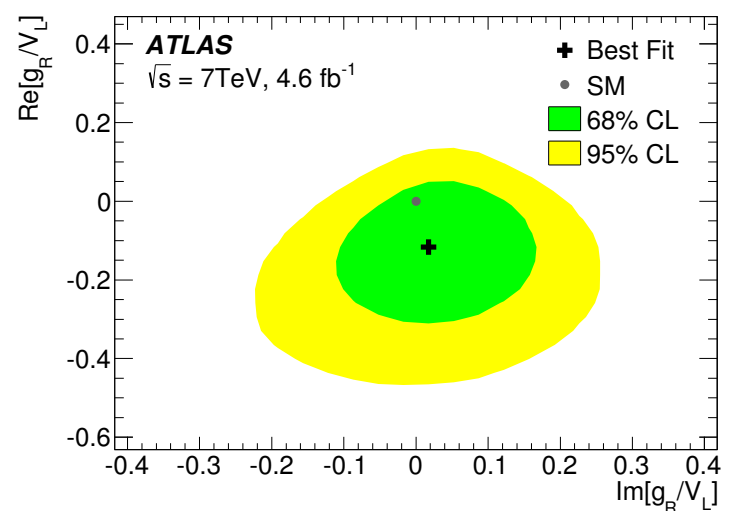

(d)

Figure 6. Projections of the likelihood function constructed from the signal region probability density Equation (7.6) and data events into (a) $f_{1}$, (b) $\delta_{-}$, (c) $f_{1}$ vs. $\delta_{-}$, and (d) $\operatorname{Re}\left[g_{\mathrm{R}} / V_{\mathrm{L}}\right]$ vs. $\operatorname{Im}\left[g_{\mathrm{R}} / V_{\mathrm{L}}\right]$, with systematic uncertainties incorporated. The black points indicate the largest evaluated likelihood in each bin of the projected variable. Gaussian fits to the one-dimensional projections were performed, displayed as the red curve. Regions shown in green and yellow represent the $68 \%$ and $95 \%$ confidence level regions, respectively. A black line or cross indicates the observed value, and the grey line or point indicates the SM expectation.

uncertainty in the top-quark, $W$ boson and $b$-quark masses [69] is $<0.01$ in $\operatorname{Re}\left[g_{\mathrm{R}} / V_{\mathrm{L}}\right]$, and $<0.0001$ in $\operatorname{Im}\left[g_{\mathrm{R}} / V_{\mathrm{L}}\right]$.

Limits are placed simultaneously on the possible complex values of the ratio of the anomalous couplings $g_{\mathrm{R}}$ and $V_{\mathrm{L}}$ at $95 \%$ C.L.,

$$
\operatorname{Re}\left[\frac{g_{\mathrm{R}}}{V_{\mathrm{L}}}\right] \in[-0.36,0.10] \text { and } \operatorname{Im}\left[\frac{g_{\mathrm{R}}}{V_{\mathrm{L}}}\right] \in[-0.17,0.23] \text {. }
$$

The best constraints on $\operatorname{Re}\left[g_{\mathrm{R}}\right]$ come from $W$ boson helicity fractions in top-quark decays, with $\operatorname{Re}\left[g_{\mathrm{R}}\right]$ of $[-0.08,0.04]$ and $[-0.08,0.07]$, both at $95 \%$ C.L., from ATLAS $[17]$ and from CMS [18], respectively. However, these limits use the measured single top-quark production cross-section $[46,70]$ along with the assumption that $V_{\mathrm{L}}=1$ and $\operatorname{Im}\left[g_{\mathrm{R}}\right]=0$. Without these assumptions no value within the range $0.0 \lesssim \operatorname{Re}\left[g_{\mathrm{R}} / V_{\mathrm{L}}\right] \lesssim 0.8$ can be 
excluded. The limits presented in this paper remove these assumptions and extend the knowledge of $g_{\mathrm{R}}$ to the whole complex plane by simultaneously measuring information about $\operatorname{Re}\left[g_{\mathrm{R}} / V_{\mathrm{L}}\right]$ and $\operatorname{Im}\left[g_{\mathrm{R}} / V_{\mathrm{L}}\right]$; the latter is previously unmeasured.

\section{Conclusion}

The analysis described in this publication is a two-dimensional measurement of anomalous couplings in the $W t b$ vertex in $t$-channel single top-quarks events, performed on $4.6 \mathrm{fb}^{-1}$ of $p p$ collisions at a centre-of-mass energy of $7 \mathrm{TeV}$ collected by the ATLAS detector at the LHC. An analytic folding model and likelihood maximisation techniques are used to extract values of the parameters $f_{1}$ and $\delta_{-}$in a parameterisation of the decay of the top quark, which translate to limits on the couplings $\operatorname{Re}\left[g_{\mathrm{R}} / V_{\mathrm{L}}\right]$ and $\operatorname{Im}\left[g_{\mathrm{R}} / V_{\mathrm{L}}\right]$ with $V_{\mathrm{R}}=g_{\mathrm{L}}=0$. The coupling parameterisation in terms of the spherical angles $\theta^{*}$ and $\phi^{*}$ defines the angular distribution of the $t$-channel signal process. Efficiency and resolution functions are fit in this space, which are then folded into the signal model describing the underlying physics analytically. A background function is fit and added to the signal model. The full model is used to construct a likelihood and its characterisation provides estimators of the best-fit central value, uncertainties, and correlations between $f_{1}$ and $\delta_{-}$, or $\operatorname{Re}\left[g_{\mathrm{R}} / V_{\mathrm{L}}\right]$ and $\operatorname{Im}\left[g_{\mathrm{R}} / V_{\mathrm{L}}\right]$. The result is combined with estimated sources of systematic uncertainty to give the measurement of the parameters, $f_{1}=0.37 \pm 0.05$ (stat.) \pm 0.05 (syst.), $\delta_{-}=-0.014 \pi \pm 0.023 \pi$ (stat.) $\pm 0.028 \pi$ (syst.), and $\rho\left(f_{1}, \delta_{-}\right)=0.15$, or $\operatorname{Re}\left[g_{\mathrm{R}} / V_{\mathrm{L}}\right]=$ $-0.13 \pm 0.07$ (stat.) \pm 0.10 (syst.), $\operatorname{Im}\left[g_{\mathrm{R}} / V_{\mathrm{L}}\right]=0.03 \pm 0.06$ (stat.) \pm 0.07 (syst.), and $\rho\left(\operatorname{Re}\left[g_{\mathrm{R}} / V_{\mathrm{L}}\right], \operatorname{Im}\left[g_{\mathrm{R}} / V_{\mathrm{L}}\right]\right)=0.11$ in terms of the couplings. The measurement sets limits at the $95 \%$ confidence level on $\operatorname{Re}\left[g_{\mathrm{R}} / V_{\mathrm{L}}\right] \in[-0.36,0.10]$ and $\operatorname{Im}\left[g_{\mathrm{R}} / V_{\mathrm{L}}\right] \in[-0.17,0.23]$. The limits on $\operatorname{Re}\left[g_{\mathrm{R}} / V_{\mathrm{L}}\right]$ are complementary to limits from $B$-meson decay and $W$ helicity measurements, and contain previously unmeasured information about $\operatorname{Im}\left[g_{\mathrm{R}} / V_{\mathrm{L}}\right]$. The measured values are in agreement with SM expectations.

\section{Acknowledgments}

We thank CERN for the very successful operation of the LHC, as well as the support staff from our institutions without whom ATLAS could not be operated efficiently.

We acknowledge the support of ANPCyT, Argentina; YerPhI, Armenia; ARC, Australia; BMWFW and FWF, Austria; ANAS, Azerbaijan; SSTC, Belarus; CNPq and FAPESP, Brazil; NSERC, NRC and CFI, Canada; CERN; CONICYT, Chile; CAS, MOST and NSFC, China; COLCIENCIAS, Colombia; MSMT CR, MPO CR and VSC CR, Czech Republic; DNRF, DNSRC and Lundbeck Foundation, Denmark; IN2P3-CNRS, CEADSM/IRFU, France; GNSF, Georgia; BMBF, HGF, and MPG, Germany; GSRT, Greece; RGC, Hong Kong SAR, China; ISF, I-CORE and Benoziyo Center, Israel; INFN, Italy; MEXT and JSPS, Japan; CNRST, Morocco; FOM and NWO, Netherlands; RCN, Norway; MNiSW and NCN, Poland; FCT, Portugal; MNE/IFA, Romania; MES of Russia and NRC KI, Russian Federation; JINR; MESTD, Serbia; MSSR, Slovakia; ARRS and MIZŠ, Slovenia; DST/NRF, South Africa; MINECO, Spain; SRC and Wallenberg Foundation, 
Sweden; SERI, SNSF and Cantons of Bern and Geneva, Switzerland; MOST, Taiwan; TAEK, Turkey; STFC, United Kingdom; DOE and NSF, United States of America. In addition, individual groups and members have received support from BCKDF, the Canada Council, CANARIE, CRC, Compute Canada, FQRNT, and the Ontario Innovation Trust, Canada; EPLANET, ERC, FP7, Horizon 2020 and Marie Skłodowska-Curie Actions, European Union; Investissements d'Avenir Labex and Idex, ANR, Region Auvergne and Fondation Partager le Savoir, France; DFG and AvH Foundation, Germany; Herakleitos, Thales and Aristeia programmes co-financed by EU-ESF and the Greek NSRF; BSF, GIF and Minerva, Israel; BRF, Norway; the Royal Society and Leverhulme Trust, United Kingdom.

The crucial computing support from all WLCG partners is acknowledged gratefully, in particular from CERN and the ATLAS Tier-1 facilities at TRIUMF (Canada), NDGF (Denmark, Norway, Sweden), CC-IN2P3 (France), KIT/GridKA (Germany), INFN-CNAF (Italy), NL-T1 (Netherlands), PIC (Spain), ASGC (Taiwan), RAL (UK) and BNL (USA) and in the Tier-2 facilities worldwide. 


\section{A Parameter dependence of the folding and background models}

The derivation of the efficiency, resolution, and background models, described in Equations (7.1)-(7.6), is based on the form of these distributions in MC simulation. For the background model, constructed from the sum of all predicted backgrounds with an appreciable effect on the distribution, this includes events containing top quarks, primarily from $t \bar{t}$ production, the distribution of which is affected by changing the values of the couplings $V_{\mathrm{L}}$ and $g_{\mathrm{R}}$. The efficiency and resolution models are averages over all unmeasured distributions in the signal. Variations in the values of $V_{\mathrm{L}}$ and $g_{\mathrm{R}}$ alter those unmeasured distributions, which could lead to a dependence on these couplings for the efficiency and resolution models. For instance, $t$-channel single top-quark production depends on anomalous coupling in both the top-quark production and decay vertices, so varying the couplings alters production-side distributions, such as the $p_{\mathrm{T}}$ and $\eta$ distributions of the top or spectator quark.

To test how these potential sources of dependence affect the models, closure tests are performed with input MC events generated at different values of the parameters $f_{1}$ and $\delta_{-}$using efficiency, resolution, and background models derived from MC events generated with SM parameter values. The $t$-channel signal and $t \bar{t}$ background events are generated with Protos for both the input MC events and the events used to derive the models. The results of these closure tests can then be compared to the values with which the input events were generated; any trend connecting them other than a line with a slope of one and an intercept of zero indicates a dependence which could bias the measurement. The results of these tests, shown in figure 7 , indicate biases in the extracted parameters.

If the biases shown are due to the mismatch between the values of $f_{1}$ and $\delta_{-}$in the efficiency, resolution, and background models, they can be removed by varying these parameters in the functions describing these models to match their values in the signal model. This is achieved by deriving these models from $t$-channel signal MC events representing each point in $\left(f_{1}, \delta_{-}\right)$independently, and calculating the likelihood using the same $f_{1}$ and $\delta_{-}$in the signal model and in the $t$-channel signal MC events. The results of these tests, shown in figure 8 , indicate that this procedure eliminates the biases shown in figure 7 . This procedure for deriving the efficiency, resolution, and background models is used in the measurement. 


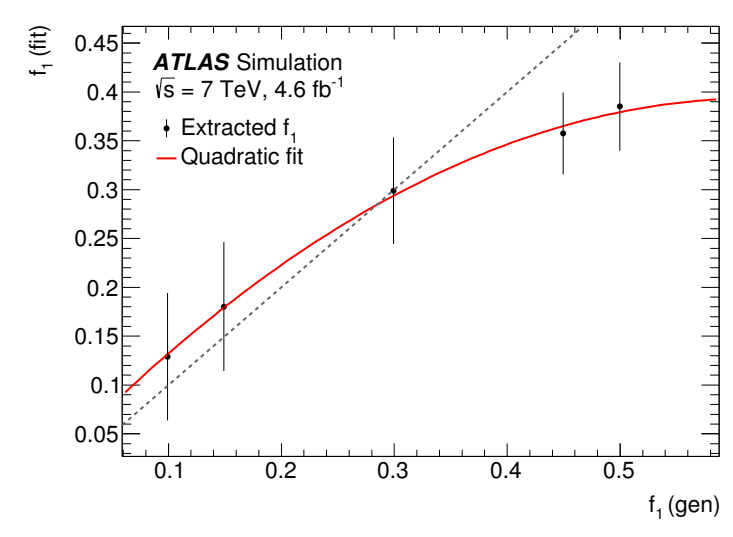

(a)

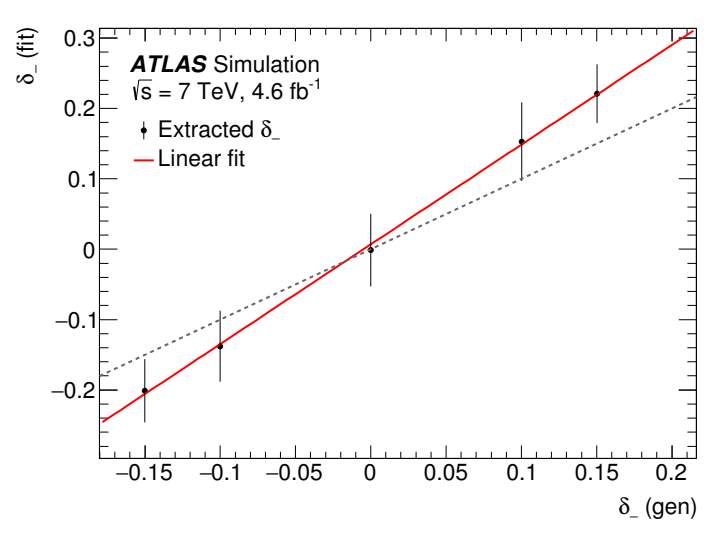

(b)

Figure 7. Biases in the estimation of the values of the parameters (a) $f_{1}$ and (b) $\delta_{-}$which arise when the efficiency, resolution, and background models are determined from SM simulated events, where $f_{1}=0.3$ and $\delta_{-}=0$. The points represent the central values obtained from the likelihood fit, with the expected statistical uncertainty in $4.6 \mathrm{fb}^{-1}$ of data at $\sqrt{s}=7 \mathrm{TeV}$ shown by the error bars. The dashed line with a slope of one and intercept of zero, representing no bias in the measurement, is added to guide the eye. A non-negligible bias is evident, and is represented at the lowest reasonable order with a polynomial fit. For $\delta_{-}$a linear fit is sufficient, while for $f_{1}$ a quadratic fit gives a better description.

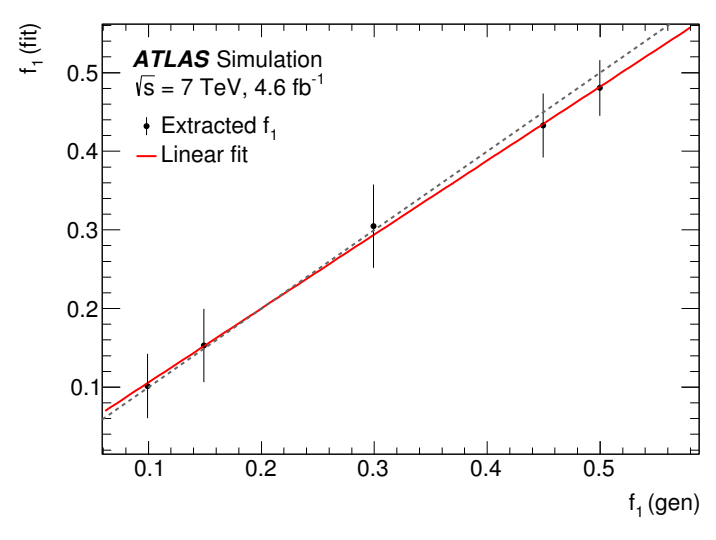

(a)

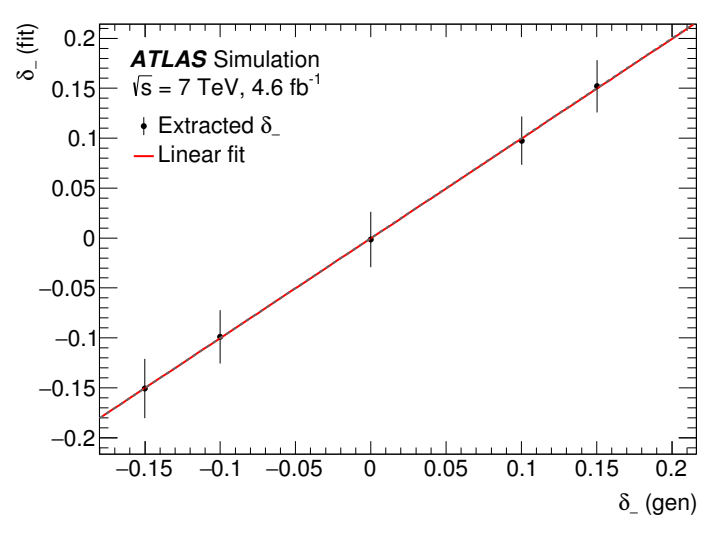

(b)

Figure 8. Comparison of the estimation of the values of the parameters (a) $f_{1}$ and (b) $\delta_{-}$derived by allowing their values in the efficiency, resolution, and background models to vary together with the values in the signal model. The points represent the central values obtained from the likelihood fit, with the expected statistical uncertainty in $4.6 \mathrm{fb}^{-1}$ of data at $\sqrt{s}=7 \mathrm{TeV}$ shown by the error bars. The dashed line with a slope of one and intercept of zero, representing no bias in the measurement, is added to guide the eye. All estimates are consistent with this line; linear fits produce a slope consistent with one and an intercept consistent with zero. 
Open Access. This article is distributed under the terms of the Creative Commons Attribution License (CC-BY 4.0), which permits any use, distribution and reproduction in any medium, provided the original author(s) and source are credited.

\section{References}

[1] CDF collaboration, F. Abe et al., Observation of top quark production in $\bar{p} p$ collisions, Phys. Rev. Lett. 74 (1995) 2626 [hep-ex/9503002] [INSPIRE].

[2] D0 collaboration, S. Abachi et al., Observation of the top quark, Phys. Rev. Lett. 74 (1995) 2632 [hep-ex/9503003] [INSPIRE].

[3] CDF collaboration, T. Aaltonen et al., First Observation of Electroweak Single Top Quark Production, Phys. Rev. Lett. 103 (2009) 092002 [arXiv:0903.0885] [INSPIRE].

[4] D0 collaboration, V.M. Abazov et al., Observation of Single Top Quark Production, Phys. Rev. Lett. 103 (2009) 092001 [arXiv:0903.0850] [INSPIRE].

[5] A.D. Martin, W.J. Stirling, R.S. Thorne and G. Watt, Parton distributions for the LHC, Eur. Phys. J. C 63 (2009) 189 [arXiv:0901.0002] [inSPIRE].

[6] N. Kidonakis, Next-to-next-to-leading-order collinear and soft gluon corrections for t-channel single top quark production, Phys. Rev. D 83 (2011) 091503 [arXiv:1103.2792] [INSPIRE].

[7] J.A. Aguilar-Saavedra, A minimal set of top anomalous couplings, Nucl. Phys. B 812 (2009) 181 [arXiv: 0811.3842] [INSPIRE].

[8] J.A. Aguilar-Saavedra, A minimal set of top-Higgs anomalous couplings, Nucl. Phys. B 821 (2009) 215 [arXiv:0904.2387] [INSPIRE].

[9] K.G. Wilson, Nonlagrangian models of current algebra, Phys. Rev. 179 (1969) 1499 [INSPIRE].

[10] W. Buchmüller and D. Wyler, Effective Lagrangian Analysis of New Interactions and Flavor Conservation, Nucl. Phys. B 268 (1986) 621 [INSPIRE].

[11] J.A. Aguilar-Saavedra and J. Bernabeu, $W$ polarisation beyond helicity fractions in top quark decays, Nucl. Phys. B 840 (2010) 349 [arXiv: 1005. 5382] [INSPIRE].

[12] B. Grzadkowski and M. Misiak, Anomalous Wtb coupling effects in the weak radiative B-meson decay, Phys. Rev. D 78 (2008) 077501 [Erratum ibid. D 84 (2011) 059903] [arXiv: 0802.1413] [INSPIRE].

[13] Q.-H. Cao, B. Yan, J.-H. Yu and C. Zhang, A general analysis of Wtb anomalous couplings, arXiv: 1504.03785 [INSPIRE].

[14] W. Bernreuther, P. Gonzalez and M. Wiebusch, The Top Quark Decay Vertex in Standard Model Extensions, Eur. Phys. J. C 60 (2009) 197 [arXiv:0812.1643] [INSPIRE].

[15] L. Duarte, G.A. González-Sprinberg and J. Vidal, Top quark anomalous tensor couplings in the two-Higgs-doublet models, JHEP 11 (2013) 114 [arXiv:1308.3652] [INSPIRE].

[16] CDF and D0 collaborations, T. Aaltonen et al., Combination of CDF and D0 measurements of the $W$ boson helicity in top quark decays, Phys. Rev. D 85 (2012) 071106 [arXiv: 1202.5272] [INSPIRE].

[17] ATLAS collaboration, Measurement of the $W$ boson polarization in top quark decays with the ATLAS detector, JHEP 06 (2012) 088 [arXiv:1205.2484] [INSPIRE]. 
[18] CMS collaboration, Measurement of the W-boson helicity in top-quark decays from $t \bar{t}$ production in lepton+jets events in pp collisions at $\sqrt{s}=7 \mathrm{TeV}$, JHEP 10 (2013) 167 [arXiv: 1308.3879] [INSPIRE].

[19] CMS collaboration, Measurement of the $W$ boson helicity in events with a single reconstructed top quark in pp collisions at $\sqrt{s}=8 \mathrm{TeV}$, JHEP 01 (2015) 053 [arXiv: 1410.1154] [INSPIRE].

[20] G. Mahlon and S.J. Parke, Improved spin basis for angular correlation studies in single top quark production at the Tevatron, Phys. Rev. D 55 (1997) 7249 [hep-ph/9611367] [INSPIRE].

[21] G. Mahlon and S.J. Parke, Single top quark production at the LHC: Understanding spin, Phys. Lett. B 476 (2000) 323 [hep-ph/9912458] [INSPIRE].

[22] R. Schwienhorst, C.P. Yuan, C. Mueller and Q.-H. Cao, Single top quark production and decay in the t-channel at next-to-leading order at the LHC, Phys. Rev. D 83 (2011) 034019 [arXiv: 1012.5132] [INSPIRE].

[23] J. Boudreau, C. Escobar, J. Mueller, K. Sapp and J. Su, Single top quark differential decay rate formulae including detector effects, arXiv:1304.5639 [INSPIRE].

[24] J.A. Aguilar-Saavedra and S.A. dos Santos, New directions for top quark polarization in the t-channel process, Phys. Rev. D 89 (2014) 114009 [arXiv: 1404.1585] [INSPIRE].

[25] J.A. Aguilar-Saavedra, Single top quark production at LHC with anomalous Wtb couplings, Nucl. Phys. B 804 (2008) 160 [arXiv:0803.3810] [INSPIRE].

[26] ATLAS collaboration, The ATLAS Experiment at the CERN Large Hadron Collider, 2008 JINST 3 S08003 [INSPIRE].

[27] ATLAS collaboration, Performance of the ATLAS Trigger System in 2010, Eur. Phys. J. C 72 (2012) 1849 [arXiv:1110.1530] [INSPIRE].

[28] L. Evans and P. Bryant, LHC Machine, 2008 JINST 3 S08001 [inSPIRE].

[29] ATLAS collaboration, Improved luminosity determination in pp collisions at $\sqrt{s}=7 \mathrm{TeV}$ using the ATLAS detector at the LHC, Eur. Phys. J. C 73 (2013) 2518 [arXiv:1302.4393] [INSPIRE].

[30] ATLAS collaboration, Performance of the ATLAS muon trigger in pp collisions at $\sqrt{s}=8 \mathrm{TeV}$, Eur. Phys. J. C 75 (2015) 120 [arXiv: 1408.3179] [InSPIRE].

[31] B.P. Kersevan and E. Richter-Was, The Monte Carlo event generator AcerMC versions 2.0 to 3.8 with interfaces to PYTHIA 6.4, HERWIG 6.5 and ARIADNE 4.1, Comput. Phys. Commun. 184 (2013) 919 [hep-ph/0405247] [INSPIRE].

[32] J. Pumplin, D.R. Stump, J. Huston, H.L. Lai, P.M. Nadolsky and W.K. Tung, New generation of parton distributions with uncertainties from global QCD analysis, JHEP 07 (2002) 012 [hep-ph/0201195] [INSPIRE].

[33] B.P. Kersevan and I. Hinchliffe, A consistent prescription for the production involving massive quarks in hadron collisions, JHEP 09 (2006) 033 [hep-ph/0603068] [INSPIRE].

[34] T. Sjöstrand, S. Mrenna and P.Z. Skands, PYTHIA 6.4 Physics and Manual, JHEP 05 (2006) 026 [hep-ph/0603175] [INSPIRE].

[35] P.Z. Skands, Tuning Monte Carlo Generators: The Perugia Tunes, Phys. Rev. D 82 (2010) 074018 [arXiv: 1005 . 3457] [INSPIRE]. 
[36] H.-L. Lai et al., New parton distributions for collider physics, Phys. Rev. D 82 (2010) 074024 [arXiv: 1007.2241] [INSPIRE].

[37] ATLAS collaboration, ATLAS tunes of PYTHIA 6 and PYTHIA 8 for MC11, ATL-PHYS-PUB-2011-009 (2011).

[38] A. Sherstnev and R.S. Thorne, Parton Distributions for LO Generators, Eur. Phys. J. C 55 (2008) 553 [arXiv:0711.2473] [INSPIRE].

[39] M.L. Mangano, M. Moretti, F. Piccinini, R. Pittau and A.D. Polosa, ALPGEN, a generator for hard multiparton processes in hadronic collisions, JHEP 07 (2003) 001 [hep-ph/0206293] [INSPIRE].

[40] G. Corcella et al., HERWIG 6: An event generator for hadron emission reactions with interfering gluons (including supersymmetric processes), JHEP 01 (2001) 010 [hep-ph/0011363] [INSPIRE].

[41] J.M. Butterworth, J.R. Forshaw and M.H. Seymour, Multiparton interactions in photoproduction at HERA, Z. Phys. C 72 (1996) 637 [hep-ph/9601371] [INSPIRE].

[42] M.L. Mangano, M. Moretti, F. Piccinini and M. Treccani, Matching matrix elements and shower evolution for top-quark production in hadronic collisions, JHEP 01 (2007) 013 [hep-ph/0611129] [INSPIRE].

[43] ATLAS collaboration, The ATLAS Simulation Infrastructure, Eur. Phys. J. C 70 (2010) 823 [arXiv: 1005.4568] [INSPIRE].

[44] GEANT4 collaboration, S. Agostinelli et al., GEANT4: A simulation toolkit, Nucl. Instrum. Meth. A 506 (2003) 250 [inSPIRE].

[45] ATLAS collaboration, The simulation principle and performance of the ATLAS fast calorimeter simulation FastCaloSim, ATL-PHYS-PUB-2010-013 (2010).

[46] ATLAS collaboration, Comprehensive measurements of $t$-channel single top-quark production cross sections at $\sqrt{s}=7 \mathrm{TeV}$ with the ATLAS detector, Phys. Rev. D 90 (2014) 112006 [arXiv: 1406 .7844] [INSPIRE].

[47] M. Beneke, P. Falgari, S. Klein and C. Schwinn, Hadronic top-quark pair production with NNLL threshold resummation, Nucl. Phys. B 855 (2012) 695 [arXiv:1109.1536] [INSPIRE].

[48] M. Cacciari, M. Czakon, M. Mangano, A. Mitov and P. Nason, Top-pair production at hadron colliders with next-to-next-to-leading logarithmic soft-gluon resummation, Phys. Lett. B 710 (2012) 612 [arXiv:1111.5869] [INSPIRE].

[49] P. Bärnreuther, M. Czakon and A. Mitov, Percent Level Precision Physics at the Tevatron: First Genuine NNLO QCD Corrections to $q \bar{q} \rightarrow t \bar{t}+X$, Phys. Rev. Lett. 109 (2012) 132001 [arXiv: 1204.5201] [INSPIRE].

[50] M. Czakon and A. Mitov, NNLO corrections to top-pair production at hadron colliders: the all-fermionic scattering channels, JHEP 12 (2012) 054 [arXiv:1207.0236] [INSPIRE].

[51] M. Czakon and A. Mitov, NNLO corrections to top pair production at hadron colliders: the quark-gluon reaction, JHEP 01 (2013) 080 [arXiv:1210.6832] [INSPIRE].

[52] M. Czakon, P. Fiedler and A. Mitov, Total Top-Quark Pair-Production Cross section at Hadron Colliders Through $O\left(\alpha \frac{4}{S}\right)$, Phys. Rev. Lett. 110 (2013) 252004 [arXiv:1303.6254] [INSPIRE]. 
[53] M. Czakon and A. Mitov, Top++: A Program for the Calculation of the Top-Pair Cross-Section at Hadron Colliders, Comput. Phys. Commun. 185 (2014) 2930 [arXiv:1112.5675] [INSPIRE].

[54] M. Botje et al., The PDF4LHC Working Group Interim Recommendations, arXiv:1101.0538 [INSPIRE].

[55] R.D. Ball et al., A first unbiased global NLO determination of parton distributions and their uncertainties, Nucl. Phys. B 838 (2010) 136 [arXiv: 1002.4407] [INSPIRE].

[56] N. Kidonakis, Two-loop soft anomalous dimensions for single top quark associated production with a $W^{-}$or $H^{-}$, Phys. Rev. D 82 (2010) 054018 [arXiv: 1005.4451] [INSPIRE].

[57] N. Kidonakis, NNLL resummation for s-channel single top quark production, Phys. Rev. D 81 (2010) 054028 [arXiv: 1001.5034] [INSPIRE].

[58] C. Anastasiou, L.J. Dixon, K. Melnikov and F. Petriello, High precision QCD at hadron colliders: Electroweak gauge boson rapidity distributions at NNLO, Phys. Rev. D 69 (2004) 094008 [hep-ph/0312266] [INSPIRE].

[59] J.M. Campbell and R.K. Ellis, MCFM for the Tevatron and the LHC, Nucl. Phys. Proc. Suppl. 205-206 (2010) 10 [arXiv:1007.3492] [INSPIRE].

[60] ATLAS collaboration, Measurements of top quark pair relative differential cross-sections with ATLAS in pp collisions at $\sqrt{s}=7$ TeV, Eur. Phys. J. C 73 (2013) 2261 [arXiv: 1207.5644] [INSPIRE].

[61] ATLAS collaboration, Measurement of the top quark pair production cross-section with ATLAS in the single lepton channel, Phys. Lett. B 711 (2012) 244 [arXiv:1201.1889] [INSPIRE].

[62] J.E. Cavanaugh, Unifying the derivations for the Akaike and corrected Akaike information criteria, Stat. Probab. Lett. 33 (1997) 201.

[63] A.R. Liddle, Information criteria for astrophysical model selection, Mon. Not. Roy. Astron. Soc. 377 (2007) L74 [astro-ph/0701113] [INSPIRE].

[64] G.E. Schwarz, Estimating the dimension of a model, Anna. Stat. 6 (1978) 461.

[65] S. Efromovich, Orthogonal series density estimation, Comput. Stat. 2 (2010) 467.

[66] ATLAS collaboration, Jet energy measurement and its systematic uncertainty in proton-proton collisions at $\sqrt{s}=7 \mathrm{TeV}$ with the ATLAS detector, Eur. Phys. J. C 75 (2015) 17 [arXiv: 1406.0076] [INSPIRE].

[67] S. Frixione and B.R. Webber, Matching NLO QCD computations and parton shower simulations, JHEP 06 (2002) 029 [hep-ph/0204244] [INSPIRE].

[68] M. Fischer, S. Groote, J.G. Korner and M.C. Mauser, Complete angular analysis of polarized top decay at O(alpha(s)), Phys. Rev. D 65 (2002) 054036 [hep-ph/0101322] [INSPIRE].

[69] Particle Data Group collaboration, K.A. Olive et al., Review of Particle Physics, Chin. Phys. C 38 (2014) 090001 [INSPIRE].

[70] CMS collaboration, Measurement of the single-top-quark t-channel cross section in pp collisions at $\sqrt{s}=7 \mathrm{TeV}$, JHEP 12 (2012) 035 [arXiv:1209.4533] [INSPIRE]. 


\section{The ATLAS collaboration}

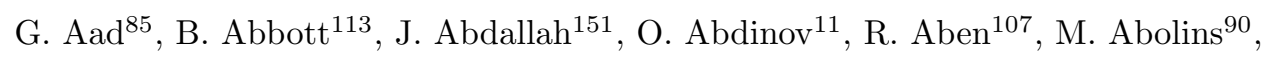
O.S. AbouZeid ${ }^{158}$, H. Abramowicz ${ }^{153}$, H. Abreu ${ }^{152}$, R. Abreu ${ }^{116}$, Y. Abulaiti ${ }^{146 a, 146 b}$, B.S. Acharya ${ }^{164 a, 164 b, a}$, L. Adamczyk ${ }^{38 a}$, D.L. Adams ${ }^{25}$, J. Adelman ${ }^{108}$, S. Adomeit ${ }^{100,}$ T. Adye ${ }^{131}$, A.A. Affolder ${ }^{74}$, T. Agatonovic-Jovin ${ }^{13}$, J. Agricola ${ }^{54}$, J.A. Aguilar-Saavedra ${ }^{126 a, 126 f}$, S.P. Ahlen ${ }^{22}$, F. Ahmadov ${ }^{65, b}$, G. Aielli ${ }^{133 a, 133 b}$, H. Akerstedt ${ }^{146 a, 146 b}$, T.P.A. Åkesson ${ }^{81}$, A.V. Akimov ${ }^{96}$, G.L. Alberghi ${ }^{20 a, 20 b}$, J. Albert ${ }^{169}$, S. Albrand ${ }^{55}$, M.J. Alconada Verzini ${ }^{71}$, M. Aleksa ${ }^{30}$, I.N. Aleksandrov ${ }^{65}$, C. Alexa ${ }^{26 b}$, G. Alexander ${ }^{153}$, T. Alexopoulos ${ }^{10}$, M. Alhroob $^{113}$, G. Alimonti ${ }^{91 a}$, L. Alio ${ }^{85}$, J. Alison ${ }^{31}$, S.P. Alkire ${ }^{35}$, B.M.M. Allbrooke ${ }^{149}$, P.P. Allport ${ }^{18}$,

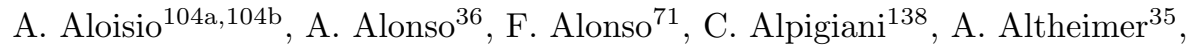

B. Alvarez Gonzalez ${ }^{30}$, D. Álvarez Piqueras ${ }^{167}$, M.G. Alviggi ${ }^{104 a, 104 b}$, B.T. Amadio ${ }^{15}$, K. Amako ${ }^{66}$, Y. Amaral Coutinho ${ }^{24 a}$, C. Amelung ${ }^{23}$, D. Amidei ${ }^{89}$, S.P. Amor Dos Santos ${ }^{126 a, 126 c}$, A. Amorim ${ }^{126 a, 126 b}$, S. Amoroso ${ }^{48}$, N. Amram ${ }^{153}$, G. Amundsen ${ }^{23}$, C. Anastopoulos ${ }^{139}$, L.S. Ancu ${ }^{49}$, N. Andari ${ }^{108}$, T. Andeen ${ }^{35}$, C.F. Anders ${ }^{58 b}$, G. Anders ${ }^{30}$, J.K. Anders ${ }^{74}$, K.J. Anderson ${ }^{31}$, A. Andreazza ${ }^{91 a, 91 b}$, V. Andrei ${ }^{58 a}$, S. Angelidakis ${ }^{9}$, I. Angelozzi ${ }^{107}$, P. Anger ${ }^{44}$, A. Angerami ${ }^{35}$, F. Anghinolfi ${ }^{30}$, A.V. Anisenkov ${ }^{109, c}$, N. Anjos ${ }^{12}$, A. Annovi ${ }^{124 a, 124 b}$, M. Antonelli ${ }^{47}$, A. Antonov ${ }^{98}$, J. Antos ${ }^{144 b}$, F. Anulli ${ }^{132 a}$, M. Aoki ${ }^{66}$, L. Aperio Bella ${ }^{18}$, G. Arabidze $^{90}$, Y. Arai ${ }^{66}$, J.P. Araque ${ }^{126 a}$, A.T.H. Arce ${ }^{45}$, F.A. Arduh ${ }^{71}$, J-F. Arguin ${ }^{95}$, S. Argyropoulos ${ }^{63}$, M. Arik ${ }^{19 a}$, A.J. Armbruster ${ }^{30}$, O. Arnaez ${ }^{30}$, H. Arnold ${ }^{48}$, M. Arratia ${ }^{28}$,

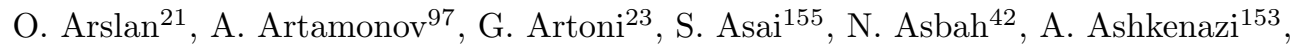

B. Åsman 146a,146b, L. Asquith ${ }^{149}$, K. Assamagan ${ }^{25}$, R. Astalos ${ }^{144 a}$, M. Atkinson ${ }^{165}$, N.B. Atlay ${ }^{141}$, K. Augsten ${ }^{128}$, M. Aurousseau ${ }^{145 b}$, G. Avolio ${ }^{30}$, B. Axen ${ }^{15}$, M.K. Ayoub ${ }^{117}$, G. Azuelos ${ }^{95, d}$, M.A. Baak ${ }^{30}$, A.E. Baas ${ }^{58 a}$, M.J. Baca ${ }^{18}$, C. Bacci'134a,134b , H. Bachacou ${ }^{136}$, K. Bachas ${ }^{154}$, M. Backes ${ }^{30}$, M. Backhaus ${ }^{30}$, P. Bagiacchi ${ }^{132 a, 132 b}$, P. Bagnaia ${ }^{132 a, 132 b}$, Y. Bai ${ }^{33 a}$, T. Bain ${ }^{35}$, J.T. Baines ${ }^{131}$, O.K. Baker ${ }^{176}$, E.M. Baldin ${ }^{109, c}$, P. Balek ${ }^{129}$, T. Balestri ${ }^{148}$, F. Balli ${ }^{84}$, W.K. Balunas ${ }^{122}$, E. Banas ${ }^{39}$, Sw. Banerjee ${ }^{173, e}$, A.A.E. Bannoura ${ }^{175}$, L. Barak ${ }^{30}$, E.L. Barberio ${ }^{88}$, D. Barberis ${ }^{50 a, 50 b}$, M. Barbero ${ }^{85}$, T. Barillari ${ }^{101}$, M. Barisonzi ${ }^{164 a, 164 b}$, T. Barklow ${ }^{143}$, N. Barlow ${ }^{28}$, S.L. Barnes ${ }^{84}$, B.M. Barnett ${ }^{131}$, R.M. Barnett ${ }^{15}$, Z. Barnovska ${ }^{5}$, A. Baroncelli ${ }^{134 a}$, G. Barone ${ }^{23}$, A.J. Barr ${ }^{120}$, F. Barreiro ${ }^{82}$, J. Barreiro Guimarães da Costa ${ }^{57}$, R. Bartoldus ${ }^{143}$, A.E. Barton ${ }^{72}$, P. Bartos ${ }^{144 a}$, A. Basalaev ${ }^{123}$, A. Bassalat ${ }^{117}$, A. Basye ${ }^{165}$, R.L. Bates ${ }^{53}$, S.J. Batista ${ }^{158}$, J.R. Batley ${ }^{28}$, M. Battaglia ${ }^{137}$, M. Bauce ${ }^{132 a, 132 b}$, F. Bauer ${ }^{136}$, H.S. Bawa ${ }^{143, f}$, J.B. Beacham ${ }^{111}$, M.D. Beattie ${ }^{72}$, T. Beau ${ }^{80}$, P.H. Beauchemin ${ }^{161}$, R. Beccherle ${ }^{124 a, 124 b}$, P. Bechtle ${ }^{21}$, H.P. Beck ${ }^{17, g}$, K. Becker ${ }^{120}$, M. Becker ${ }^{83}$, M. Beckingham ${ }^{170}$, C. Becot ${ }^{117}$, A.J. Beddall ${ }^{19 b}$, A. Beddall ${ }^{19 b}$, V.A. Bednyakov ${ }^{65}$, C.P. Bee ${ }^{148}$, L.J. Beemster ${ }^{107}$, T.A. Beermann ${ }^{30}$, M. Begel ${ }^{25}$, J.K. Behr ${ }^{120}$, C. Belanger-Champagne ${ }^{87}$, W.H. Bell ${ }^{49}$, G. Bella ${ }^{153}$, L. Bellagamba ${ }^{20 a}$, A. Bellerive ${ }^{29}$, M. Bellomo ${ }^{86}$, K. Belotskiy ${ }^{98}$, O. Beltramello ${ }^{30}$, O. Benary ${ }^{153}$, D. Benchekroun ${ }^{135 a}$, M. Bender ${ }^{100}$, K. Bendtz ${ }^{146 a, 146 b}$, N. Benekos ${ }^{10}$, Y. Benhammou ${ }^{153}$, E. Benhar Noccioli ${ }^{49}$, J.A. Benitez Garcia ${ }^{159 b}$, D.P. Benjamin ${ }^{45}$, J.R. Bensinger ${ }^{23}$, S. Bentvelsen ${ }^{107}$, L. Beresford ${ }^{120}$, M. Beretta ${ }^{47}$, D. Berge ${ }^{107}$, E. Bergeaas Kuutmann ${ }^{166}$, N. Berger ${ }^{5}$, F. Berghaus ${ }^{169}$, J. Beringer ${ }^{15}$, C. Bernard ${ }^{22}$, N.R. Bernard ${ }^{86}$, C. Bernius ${ }^{110}$, F.U. Bernlochner ${ }^{21}$, T. Berry ${ }^{77}$, P. Berta ${ }^{129}$, C. Bertella ${ }^{83}$, G. Bertoli ${ }^{146 a}, 146 b$, F. Bertolucci ${ }^{124 a}, 124 b$, C. Bertsche ${ }^{113}$, D. Bertsche ${ }^{113}$, M.I. Besana ${ }^{91 a}$, G.J. Besjes ${ }^{36}$, O. Bessidskaia Bylund ${ }^{146 a, 146 b}$, M. Bessner ${ }^{42}$, N. Besson ${ }^{136}$, C. Betancourt ${ }^{48}$, S. Bethke ${ }^{101}$, A.J. Bevan ${ }^{76}$, W. Bhimji ${ }^{15}$, R.M. Bianchi ${ }^{125}$, L. Bianchini ${ }^{23}$, M. Bianco ${ }^{30}$, O. Biebel ${ }^{100}$, D. Biedermann ${ }^{16}$, N.V. Biesuz ${ }^{124 a, 124 b}$, M. Biglietti134a ${ }^{13}$ J. Bilbao De Mendizabal ${ }^{49}$, H. Bilokon ${ }^{47}$, M. Bindi ${ }^{54}$, S. Binet ${ }^{117}$, A. Bingul ${ }^{19 b}$, C. Bini ${ }^{132 a, 132 b}$, S. Biondi ${ }^{20 a, 20 b}$, D.M. Bjergaard ${ }^{45}$, C.W. Black ${ }^{150}$, J.E. Black ${ }^{143}$, K.M. Black ${ }^{22}$, D. Blackburn ${ }^{138}$, R.E. Blair ${ }^{6}$, J.-B. Blanchard ${ }^{136}$, 
J.E. Blanco ${ }^{77}$, T. Blazek ${ }^{144 a}$, I. Bloch ${ }^{42}$, C. Blocker ${ }^{23}$, W. Blum ${ }^{83, *}$, U. Blumenschein ${ }^{54}$, S. Blunier ${ }^{32 a}$, G.J. Bobbink ${ }^{107}$, V.S. Bobrovnikov ${ }^{109, c}$, S.S. Bocchetta ${ }^{81}$, A. Bocci ${ }^{45}$, C. Bock ${ }^{100}$, M. Boehler ${ }^{48}$, J.A. Bogaerts ${ }^{30}$, D. Bogavac ${ }^{13}$, A.G. Bogdanchikov ${ }^{109}$, C. Bohm ${ }^{146 a}$, V. Boisvert ${ }^{77}$, T. Bold ${ }^{38 a}$, V. Boldea ${ }^{26 b}$, A.S. Boldyrev ${ }^{99}$, M. Bomben ${ }^{80}$, M. Bona ${ }^{76}$, M. Boonekamp ${ }^{136}$, A. Borisov ${ }^{130}$, G. Borissov ${ }^{72}$, S. Borroni ${ }^{42}$, J. Bortfeldt ${ }^{100}$, V. Bortolotto ${ }^{60 a, 60 b, 60 c}$, K. Bos $^{107}$, D. Boscherini ${ }^{20 a}$, M. Bosman ${ }^{12}$, J. Boudreau ${ }^{125}$, J. Bouffard ${ }^{2}$, E.V. Bouhova-Thacker ${ }^{72}$, D. Boumediene ${ }^{34}$, C. Bourdarios ${ }^{117}$, N. Bousson ${ }^{114}$, S.K. Boutle ${ }^{53}$, A. Boveia ${ }^{30}$, J. Boyd ${ }^{30}$, I.R. Boyko ${ }^{65}$, I. Bozic ${ }^{13}$, J. Bracinik ${ }^{18}$, A. Brandt ${ }^{8}$, G. Brandt ${ }^{54}$, O. Brandt ${ }^{58 a}$, U. Bratzler ${ }^{156}$, B. Brau ${ }^{86}$, J.E. Brau ${ }^{116}$, H.M. Braun ${ }^{175, *}$, W.D. Breaden Madden ${ }^{53}$, K. Brendlinger ${ }^{122}$, A.J. Brennan ${ }^{88}$, L. Brenner ${ }^{107}$, R. Brenner ${ }^{166}$, S. Bressler ${ }^{172}$, T.M. Bristow ${ }^{46}$, D. Britton ${ }^{53}$, D. Britzger ${ }^{42}$, F.M. Brochu ${ }^{28}$, I. Brock ${ }^{21}$, R. Brock ${ }^{90}$, J. Bronner ${ }^{101}$, G. Brooijmans ${ }^{35}$,

T. Brooks ${ }^{77}$, W.K. Brooks ${ }^{32 b}$, J. Brosamer ${ }^{15}$, E. Brost ${ }^{116}$, P.A. Bruckman de Renstrom ${ }^{39}$, D. Bruncko ${ }^{144 b}$, R. Bruneliere ${ }^{48}$, A. Bruni ${ }^{20 a}$, G. Bruni ${ }^{20 a}$, M. Bruschi $^{20 a}$, N. Bruscino ${ }^{21}$, L. Bryngemark ${ }^{81}$, T. Buanes ${ }^{14}$, Q. Buat ${ }^{142}$, P. Buchholz ${ }^{141}$, A.G. Buckley ${ }^{53}$, I.A. Budagov ${ }^{65}$, F. Buehrer ${ }^{48}$, L. Bugge ${ }^{119}$, M.K. Bugge ${ }^{119}$, O. Bulekov ${ }^{98}$, D. Bullock ${ }^{8}$, H. Burckhart ${ }^{30}$, S. Burdin ${ }^{74}$, C.D. Burgard ${ }^{48}$, B. Burghgrave ${ }^{108}$, S. Burke ${ }^{131}$, I. Burmeister ${ }^{43}$, E. Busato ${ }^{34}$, D. Büscher ${ }^{48}$, V. Büscher ${ }^{83}$, P. Bussey ${ }^{53}$, J.M. Butler ${ }^{22}$, A.I. Butt ${ }^{3}$, C.M. Buttar ${ }^{53}$, J.M. Butterworth ${ }^{78}$, P. Butti ${ }^{107}$, W. Buttinger ${ }^{25}$, A. Buzatu ${ }^{53}$, A.R. Buzykaev ${ }^{109, c}$, S. Cabrera Urbán ${ }^{167}$, D. Caforio ${ }^{128}$, V.M. Cairo ${ }^{37 a, 37 b}$, O. Cakir ${ }^{4 a}$, N. Calace $^{49}$, P. Calafiura ${ }^{15}$, A. Calandri ${ }^{136}$, G. Calderini ${ }^{80}$, P. Calfayan ${ }^{100}$, L.P. Caloba ${ }^{24 a}$, D. Calvet $^{34}$, S. Calvet ${ }^{34}$,

R. Camacho Toro ${ }^{31}$, S. Camarda ${ }^{42}$, P. Camarri ${ }^{133 a, 133 b}$, D. Cameron ${ }^{119}$,

R. Caminal Armadans ${ }^{165}$, S. Campana ${ }^{30}$, M. Campanelli ${ }^{78}$, A. Campoverde ${ }^{148}$, V. Canale ${ }^{104 a, 104 b}$, A. Canepa ${ }^{159 a}$, M. Cano Bret ${ }^{33 e}$, J. Cantero $^{82}$, R. Cantrill $^{126 a}$, T. Cao $^{40}$, M.D.M. Capeans Garrido ${ }^{30}$, I. Caprini ${ }^{26 b}$, M. Caprini ${ }^{26 b}$, M. Capua ${ }^{37 a, 37 b}$, R. Caputo ${ }^{83}$, R.M. Carbone ${ }^{35}$, R. Cardarelli ${ }^{133 a}$, F. Cardillo ${ }^{48}$, T. Carli ${ }^{30}$, G. Carlino ${ }^{104 a}$, L. Carminati ${ }^{91 a, 91 b}$, S. Caron ${ }^{106}$, E. Carquin ${ }^{32 a}$, G.D. Carrillo-Montoya ${ }^{30}$, J.R. Carter ${ }^{28}$, J. Carvalho ${ }^{126 a, 126 c}$, D. Casadei ${ }^{78}$, M.P. Casado ${ }^{12}$, M. Casolino ${ }^{12}$, D.W. Casper ${ }^{163}$, E. Castaneda-Miranda ${ }^{145 a}$, A. Castelli ${ }^{107}$, V. Castillo Gimenez ${ }^{167}$, N.F. Castro ${ }^{126 a}, h$, P. Catastini $^{57}$, A. Catinaccio $^{30}$, J.R. Catmore ${ }^{119}$, A. Cattai ${ }^{30}$, J. Caudron ${ }^{83}$, V. Cavaliere ${ }^{165}$, D. Cavalli ${ }^{91 a}$, M. Cavalli-Sforza ${ }^{12}$, V. Cavasinni ${ }^{124 a, 124 b}$, F. Ceradini134a,134b L. Cerda Alberich ${ }^{167}$, B.C. Cerio ${ }^{45}$, K. Cerny ${ }^{129}$, A.S. Cerqueira ${ }^{24 b}$, A. Cerri ${ }^{149}$, L. Cerrito ${ }^{76}$, F. Cerutti ${ }^{15}$, M. Cerv ${ }^{30}$, A. Cervelli ${ }^{17}$, S.A. Cetin ${ }^{19 c}$, A. Chafaq ${ }^{135 a}$, D. Chakraborty ${ }^{108}$, I. Chalupkova ${ }^{129}$, Y.L. Chan ${ }^{60 a}$, P. Chang $^{165}$, J.D. Chapman ${ }^{28}$, D.G. Charlton ${ }^{18}$, C.C. Chau ${ }^{158}$, C.A. Chavez Barajas ${ }^{149}$, S. Cheatham ${ }^{152}$, A. Chegwidden ${ }^{90}$, S. Chekanov ${ }^{6}$, S.V. Chekulaev ${ }^{159 a}$, G.A. Chelkov ${ }^{65, i}$, M.A. Chelstowska ${ }^{89}$, C. Chen ${ }^{64}$, H. Chen ${ }^{25}$, K. Chen ${ }^{148}$, L. Chen ${ }^{33 d, j}$, S. Chen ${ }^{33 c}$, S. Chen ${ }^{155}$, X. Chen ${ }^{33 f}$, Y. Chen ${ }^{67}$, H.C. Cheng ${ }^{89}$, Y. Cheng ${ }^{31}$, A. Cheplakov ${ }^{65}$, E. Cheremushkina ${ }^{130}$, R. Cherkaoui El Moursli ${ }^{135 e}$, V. Chernyatin ${ }^{25, *}$, E. Cheu ${ }^{7}$, L. Chevalier ${ }^{136}$, V. Chiarella ${ }^{47}$, G. Chiarelli ${ }^{124 a, 124 b}$, G. Chiodini ${ }^{73 a}$, A.S. Chisholm ${ }^{18}$, R.T. Chislett ${ }^{78}$, A. Chitan ${ }^{26 b}$, M.V. Chizhov ${ }^{65}$, K. Choi ${ }^{61}$, S. Chouridou ${ }^{9}$, B.K.B. Chow ${ }^{100}$, V. Christodoulou ${ }^{78}$, D. Chromek-Burckhart ${ }^{30}$, J. Chudoba ${ }^{127}$, A.J. Chuinard ${ }^{87}$, J.J. Chwastowski ${ }^{39}$, L. Chytka ${ }^{115}$, G. Ciapetti ${ }^{132 a, 132 b}$, A.K. Ciftci $^{4 a}$, D. Cinca ${ }^{53}$, V. Cindro ${ }^{75}$, I.A. Cioara ${ }^{21}$, A. Ciocio ${ }^{15}$, F. Cirotto ${ }^{104 a, 104 b}$, Z.H. Citron ${ }^{172}$, M. Ciubancan ${ }^{26 b}$, A. Clark ${ }^{49}$, B.L. Clark ${ }^{57}$, P.J. Clark ${ }^{46}$, R.N. Clarke ${ }^{15}$, C. Clement ${ }^{146 a, 146 b}$, Y. Coadou ${ }^{85}$, M. Cobal ${ }^{164 a, 164 c}$, A. Coccaro ${ }^{49}$, J. Cochran ${ }^{64}$, L. Coffey ${ }^{23}$, J.G. Cogan $^{143}$, L. Colasurdo ${ }^{106}$, B. Cole ${ }^{35}$, S. Cole ${ }^{108}$, A.P. Colijn ${ }^{107}$, J. Collot ${ }^{55}$, T. Colombo ${ }^{58 c}$, G. Compostella ${ }^{101}$, P. Conde Muiño ${ }^{126 a, 126 b}$,

E. Coniavitis ${ }^{48}$, S.H. Connell ${ }^{145 b}$, I.A. Connelly ${ }^{77}$, V. Consorti ${ }^{48}$, S. Constantinescu ${ }^{26 b}$, C. Conta ${ }^{121 \mathrm{a}, 121 \mathrm{~b}}$, G. Conti ${ }^{30}$, F. Conventi ${ }^{104 \mathrm{a}, k}$, M. Cooke ${ }^{15}$, B.D. Cooper ${ }^{78}$, A.M. Cooper-Sarkar ${ }^{120}$, T. Cornelissen ${ }^{175}$, M. Corradi ${ }^{20 a}$, F. Corriveau ${ }^{87, l}$, A. Corso-Radu ${ }^{163}$, A. Cortes-Gonzalez ${ }^{12}$, G. Cortiana ${ }^{101}$, G. Costa ${ }^{91 a}$, M.J. Costa ${ }^{167}$, D. Costanzo $^{139}$, D. Côté ${ }^{\text {, }}$ 


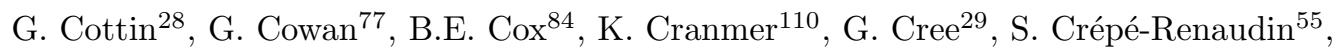

F. Crescioli ${ }^{80}$, W.A. Cribbs ${ }^{146 a, 146 b}$, M. Crispin Ortuzar ${ }^{120}$, M. Cristinziani ${ }^{21}$, V. Croft ${ }^{106}$,

G. Crosettiina,37b, T. Cuhadar Donszelmann ${ }^{139}$, J. Cummings ${ }^{176}$, M. Curatolo ${ }^{47}$, J. Cúth ${ }^{83}$,

C. Cuthbert ${ }^{150}$, H. Czirr ${ }^{141}$, P. Czodrowski ${ }^{3}$, S. D'Auria ${ }^{53}$, M. D'Onofrio ${ }^{74}$,

M.J. Da Cunha Sargedas De Sousa ${ }^{126 a, 126 b}$, C. Da Via ${ }^{84}$, W. Dabrowski ${ }^{38 a}$, A. Dafinca ${ }^{120}$,

T. Dai ${ }^{89}$, O. Dale ${ }^{14}$, F. Dallaire ${ }^{95}$, C. Dallapiccola ${ }^{86}$, M. Dam $^{36}$, J.R. Dandoy ${ }^{31}$, N.P. Dang ${ }^{48}$,

A.C. Daniells ${ }^{18}$, M. Danninger ${ }^{168}$, M. Dano Hoffmann ${ }^{136}$, V. Dao ${ }^{48}$, G. Darbo ${ }^{50 a}$, S. Darmora ${ }^{8}$,

J. Dassoulas ${ }^{3}$, A. Dattagupta ${ }^{61}$, W. Davey ${ }^{21}$, C. David ${ }^{169}$, T. Davidek ${ }^{129}$, E. Davies ${ }^{120, m}$,

M. Davies ${ }^{153}$, P. Davison ${ }^{78}$, Y. Davygora ${ }^{58 a}$, E. Dawe ${ }^{88}$, I. Dawson ${ }^{139}$,

R.K. Daya-Ishmukhametova ${ }^{86}, K . \mathrm{De}^{8}$, R. de Asmundis ${ }^{104 a}$, A. De Benedetti ${ }^{113}$,

S. De Castro ${ }^{20 a, 20 b}$, S. De Cecco $^{80}$, N. De Groot ${ }^{106}$, P. de Jong ${ }^{107}$, H. De la Torre ${ }^{82}$,

F. De Lorenzi ${ }^{64}$, D. De Pedis ${ }^{132 a}$, A. De Salvo ${ }^{132 a}$, U. De Sanctis ${ }^{149}$, A. De Santo ${ }^{149}$,

J.B. De Vivie De Regie ${ }^{117}$, W.J. Dearnaley ${ }^{72}$, R. Debbe ${ }^{25}$, C. Debenedetti ${ }^{137}$, D.V. Dedovich ${ }^{65}$,

I. Deigaard ${ }^{107}$, J. Del Peso ${ }^{82}$, T. Del Prete ${ }^{124 a, 124 b}$, D. Delgove ${ }^{117}$, F. Deliot ${ }^{136}$, C.M. Delitzsch ${ }^{49}$,

M. Deliyergiyev ${ }^{75}$, A. Dell'Acqua ${ }^{30}$, L. Dell'Asta ${ }^{22}$, M. Dell'Orso ${ }^{124 a, 124 b}$, M. Della Pietra ${ }^{104 a}, k$,

D. della Volpe ${ }^{49}$, M. Delmastro ${ }^{5}$, P.A. Delsart ${ }^{55}$, C. Deluca ${ }^{107}$, D.A. DeMarco ${ }^{158}$, S. Demers ${ }^{176}$,

M. Demichev ${ }^{65}$, A. Demilly ${ }^{80}$, S.P. Denisov ${ }^{130}$, D. Derendarz ${ }^{39}$, J.E. Derkaoui ${ }^{135 d}$, F. Derue ${ }^{80}$,

P. Dervan ${ }^{74}$, K. Desch ${ }^{21}$, C. Deterre ${ }^{42}$, K. Dette ${ }^{43}$, P.O. Deviveiros ${ }^{30}$, A. Dewhurst ${ }^{131}$,

S. Dhaliwal ${ }^{23}$, A. Di Ciaccio ${ }^{133 a, 133 b}$, L. Di Ciaccio ${ }^{5}$, A. Di Domenico ${ }^{132 a, 132 b}$,

C. Di Donato ${ }^{104 a}$,104b, A. Di Girolamo ${ }^{30}$, B. Di Girolamo ${ }^{30}$, A. Di Mattia ${ }^{152}$, B. Di Micco ${ }^{134 a, 134 b}$,

R. Di Nardo ${ }^{47}$, A. Di Simone ${ }^{48}$, R. Di Sipio ${ }^{158}$, D. Di Valentino ${ }^{29}$, C. Diaconu ${ }^{85}$, M. Diamond ${ }^{158}$,

F.A. Dias ${ }^{46}$, M.A. Diaz ${ }^{32 a}$, E.B. Diehl ${ }^{89}$, J. Dietrich ${ }^{16}$, S. Diglio ${ }^{85}$, A. Dimitrievska ${ }^{13}$,

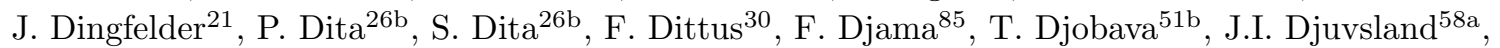
M.A.B. do Vale ${ }^{24 c}$, D. Dobos ${ }^{30}$, M. Dobre ${ }^{26 b}$, C. Doglioni ${ }^{81}$, T. Dohmae ${ }^{155}$, J. Dolejsi ${ }^{129}$,

Z. Dolezal ${ }^{129}$, B.A. Dolgoshein ${ }^{98, *}$, M. Donadelli24d ${ }^{24}$ S. Donati ${ }^{124 a, 124 b}$, P. Dondero ${ }^{121 a, 121 b}$,

J. Donini ${ }^{34}$, J. Dopke ${ }^{131}$, A. Doria ${ }^{104 a}$, M.T. Dova ${ }^{71}$, A.T. Doyle ${ }^{53}$, E. Drechsler ${ }^{54}$, M. Dris ${ }^{10}$,

Y. Du ${ }^{33 d}$, E. Dubreuil ${ }^{34}$, E. Duchovni ${ }^{172}$, G. Duckeck ${ }^{100}$, O.A. Ducu ${ }^{26 b, 85}$, D. Duda ${ }^{107}$,

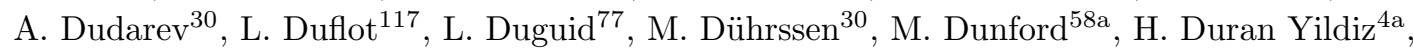

M. Düren ${ }^{52}$, A. Durglishvilis1b, D. Duschinger ${ }^{44}$, B. Dutta ${ }^{42}$, M. Dyndal ${ }^{38 a}$, C. Eckardtt ${ }^{42}$,

K.M. Ecker ${ }^{101}$, R.C. Edgar ${ }^{89}$, W. Edson ${ }^{2}$, N.C. Edwards ${ }^{46}$, W. Ehrenfeld ${ }^{21}$, T. Eifert ${ }^{30}$,

G. Eigen ${ }^{14}$, K. Einsweiler ${ }^{15}$, T. Ekelof ${ }^{166}$, M. El Kacimi ${ }^{135 c}$, M. Ellert ${ }^{166}$, S. Elles ${ }^{5}$,

F. Ellinghaus ${ }^{175}$, A.A. Elliot ${ }^{169}$, N. Ellis $^{30}$, J. Elmsheuser ${ }^{100}$, M. Elsing ${ }^{30}$, D. Emeliyanov ${ }^{131}$,

Y. Enari ${ }^{155}$, O.C. Endner ${ }^{83}$, M. Endo ${ }^{118}$, J. Erdmann ${ }^{43}$, A. Ereditato ${ }^{17}$, G. Ernis ${ }^{175}$, J. Ernst ${ }^{2}$,

M. Ernst ${ }^{25}$, S. Errede ${ }^{165}$, E. Ertel ${ }^{83}$, M. Escalier ${ }^{117}$, H. Esch ${ }^{43}$, C. Escobar ${ }^{125}$, B. Esposito ${ }^{47}$,

A.I. Etienvre ${ }^{136}$, E. Etzion ${ }^{153}$, H. Evans ${ }^{61}$, A. Ezhilov ${ }^{123}$, L. Fabbri' ${ }^{20 a, 20 b}$, G. Facini ${ }^{31}$,

R.M. Fakhrutdinov ${ }^{130}$, S. Falciano ${ }^{132 a}$, R.J. Falla ${ }^{78}$, J. Faltova ${ }^{129}$, Y. Fang ${ }^{33 a}$, M. Fanti ${ }^{91 a, 91 b}$,

A. Farbin ${ }^{8}$, A. Farilla ${ }^{134 a}$, T. Farooque ${ }^{12}$, S. Farrell ${ }^{15}$, S.M. Farrington ${ }^{170}$, P. Farthouat ${ }^{30}$,

F. Fassi ${ }^{135 e}$, P. Fassnacht ${ }^{30}$, D. Fassouliotis ${ }^{9}$, M. Faucci Giannelli 77 , A. Favareto ${ }^{50 a, 50 b}$,

L. Fayard ${ }^{117}$, O.L. Fedin ${ }^{123, n}$, W. Fedorko ${ }^{168}$, S. Feigl ${ }^{30}$, L. Feligioni ${ }^{85}$, C. Feng ${ }^{33 d}$, E.J. Feng ${ }^{30}$,

H. Feng ${ }^{89}$, A.B. Fenyuk ${ }^{130}$, L. Feremenga ${ }^{8}$, P. Fernandez Martinez ${ }^{167}$, S. Fernandez Perez ${ }^{30}$,

J. Ferrando ${ }^{53}$, A. Ferrari ${ }^{166}$, P. Ferrari ${ }^{107}$, R. Ferrari ${ }^{121 a}$, D.E. Ferreira de Lima ${ }^{53}$, A. Ferrer ${ }^{167}$,

D. Ferrere ${ }^{49}$, C. Ferretti ${ }^{89}$, A. Ferretto Parodi ${ }^{50 a, 50 b}$, M. Fiascaris ${ }^{31}$, F. Fiedler ${ }^{83}$, A. Filipčic ${ }^{75}$,

M. Filipuzzi ${ }^{42}$, F. Filthaut ${ }^{106}$, M. Fincke-Keeler ${ }^{169}$, K.D. Finelli ${ }^{150}$, M.C.N. Fiolhais ${ }^{126 a, 126 c}$,

L. Fiorini ${ }^{167}$, A. Firan ${ }^{40}$, A. Fischer ${ }^{2}$, C. Fischer ${ }^{12}$, J. Fischer ${ }^{175}$, W.C. Fisher ${ }^{90}$, N. Flaschel ${ }^{42}$,

I. Fleck ${ }^{141}$, P. Fleischmann ${ }^{89}$, G.T. Fletcher ${ }^{139}$, G. Fletcher ${ }^{76}$, R.R.M. Fletcher ${ }^{122}$, T. Flick ${ }^{175}$,

A. Floderus ${ }^{81}$, L.R. Flores Castillo ${ }^{60 a}$, M.J. Flowerdew ${ }^{101}$, A. Formica ${ }^{136}$, A. Forti ${ }^{84}$,

D. Fournier ${ }^{117}$, H. Fox ${ }^{72}$, S. Fracchia ${ }^{12}$, P. Francavilla ${ }^{80}$, M. Franchini ${ }^{20 a}$,20b , D. Francis ${ }^{30}$,

L. Franconi ${ }^{119}$, M. Franklin ${ }^{57}$, M. Frate ${ }^{163}$, M. Fraternali ${ }^{121 a, 121 b}$, D. Freeborn ${ }^{78}$, S.T. French ${ }^{28}$, 
S.M. Fressard-Batraneanu ${ }^{30}$, F. Friedrich ${ }^{44}$, D. Froidevaux ${ }^{30}$, J.A. Frost ${ }^{120}$, C. Fukunaga ${ }^{156}$, E. Fullana Torregrosa ${ }^{83}$, B.G. Fulsom ${ }^{143}$, T. Fusayasu ${ }^{102}$, J. Fuster ${ }^{167}$, C. Gabaldon ${ }^{55}$,

O. Gabizon ${ }^{175}$, A. Gabrielli ${ }^{20 a, 20 b}$, A. Gabrielli ${ }^{15}$, G.P. Gach ${ }^{18}$, S. Gadatsch ${ }^{30}$, S. Gadomski ${ }^{49}$,

G. Gagliardi ${ }^{50 a, 50 b}$, P. Gagnon ${ }^{61}$, C. Galea ${ }^{106}$, B. Galhardo ${ }^{126 a, 126 c}$, E.J. Gallas ${ }^{120}$, B.J. Gallop ${ }^{131}$,

P. Gallus ${ }^{128}$, G. Galster ${ }^{36}$, K.K. Gan ${ }^{111}$, J. Gao ${ }^{33 b, 85}$, Y. Gao ${ }^{46}$, Y.S. Gao ${ }^{143, f}$,

F.M. Garay Walls ${ }^{46}$, F. Garberson ${ }^{176}$, C. García ${ }^{167}$, J.E. García Navarro ${ }^{167}$, M. Garcia-Sciveres ${ }^{15}$,

R.W. Gardner ${ }^{31}$, N. Garelli ${ }^{143}$, V. Garonne ${ }^{119}$, C. Gatti ${ }^{47}$, A. Gaudiello ${ }^{50 a, 50 b}$, G. Gaudio ${ }^{121 a}$,

B. Gaur ${ }^{141}$, L. Gauthier ${ }^{95}$, P. Gauzzi1 ${ }^{132 a, 132 b}$, I.L. Gavrilenko ${ }^{96}$, C. Gay ${ }^{168}$, G. Gaycken ${ }^{21}$,

E.N. Gazis ${ }^{10}$, P. Ge ${ }^{33 d}$, Z. Gecse ${ }^{168}$, C.N.P. Gee ${ }^{131}$, Ch. Geich-Gimbel ${ }^{21}$, M.P. Geisler ${ }^{58 a}$,

C. Gemme ${ }^{50 a}$, M.H. Genest ${ }^{55}$, C. Geng ${ }^{33 b, o}$, S. Gentile ${ }^{132 a, 132 b}$, M. George ${ }^{54}$, S. George ${ }^{77}$,

D. Gerbaudo ${ }^{163}$, A. Gershon ${ }^{153}$, S. Ghasemi ${ }^{141}$, H. Ghazlane ${ }^{135 b}$, B. Giacobbe ${ }^{20 a}$,

S. Giagu ${ }^{132 a, 132 b}$, V. Giangiobbe ${ }^{12}$, P. Giannetti ${ }^{124 a, 124 b}$, B. Gibbard ${ }^{25}$, S.M. Gibson ${ }^{77}$,

M. Gignac ${ }^{168}$, M. Gilchriese ${ }^{15}$, T.P.S. Gillam ${ }^{28}$, D. Gillberg ${ }^{30}$, G. Gilles ${ }^{34}$, D.M. Gingrich ${ }^{3, d}$,

N. Giokaris ${ }^{9}$, M.P. Giordani1 ${ }^{164 a, 164 c}$, F.M. Giorgi ${ }^{20 a}$, F.M. Giorgi ${ }^{16}$, P.F. Giraud ${ }^{136}$, P. Giromini ${ }^{47}$,

D. Giugni ${ }^{91 a}$, C. Giuliani ${ }^{101}$, M. Giulini ${ }^{58 b}$, B.K. Gjelsten ${ }^{119}$, S. Gkaitatzis ${ }^{154}$, I. Gkialas ${ }^{154}$,

E.L. Gkougkousis ${ }^{117}$, L.K. Gladilin ${ }^{99}$, C. Glasman ${ }^{82}$, J. Glatzer ${ }^{30}$, P.C.F. Glaysher ${ }^{46}$, A. Glazov ${ }^{42}$,

M. Goblirsch-Kolb ${ }^{101}$, J.R. Goddard ${ }^{76}$, J. Godlewski ${ }^{39}$, S. Goldfarb ${ }^{89}$, T. Golling ${ }^{49}$,

D. Golubkov ${ }^{130}$, A. Gomes ${ }^{126 a, 126 b, 126 d}$, R. Gonçalo ${ }^{126 a}$, J. Goncalves Pinto Firmino Da Costa ${ }^{136}$,

L. Gonella ${ }^{21}$, S. González de la Hoz ${ }^{167}$, G. Gonzalez Parra ${ }^{12}$, S. Gonzalez-Sevilla ${ }^{49}$, L. Goossens ${ }^{30}$, P.A. Gorbounov ${ }^{97}$, H.A. Gordon ${ }^{25}$, I. Gorelov ${ }^{105}$, B. Gorini ${ }^{30}$, E. Gorini ${ }^{73 a, 73 b}$, A. Gorišek ${ }^{75}$,

E. Gornicki ${ }^{39}$, A.T. Goshaw ${ }^{45}$, C. Gössling ${ }^{43}$, M.I. Gostkin ${ }^{65}$, D. Goujdami ${ }^{135 c}$, A.G. Goussiou ${ }^{138}$, N. Govender ${ }^{145 b}$, E. Gozani ${ }^{152}$, H.M.X. Grabas ${ }^{137}$, L. Graber ${ }^{54}$, I. Grabowska-Bold ${ }^{38 a}$, P.O.J. Gradin ${ }^{166}$, P. Grafström ${ }^{20 a, 20 b}$, J. Gramling ${ }^{49}$, E. Gramstad ${ }^{119}$, S. Grancagnolo ${ }^{16}$,

V. Gratchev ${ }^{123}$, H.M. Gray ${ }^{30}$, E. Graziani ${ }^{134 a}$, Z.D. Greenwood ${ }^{79, p}$, C. Grefe ${ }^{21}$, K. Gregersen ${ }^{78}$, I.M. Gregor ${ }^{42}$, P. Grenier ${ }^{143}$, J. Griffiths ${ }^{8}$, A.A. Grillo ${ }^{137}$, K. Grimm ${ }^{72}$, S. Grinstein ${ }^{12, q}$,

Ph. Gris ${ }^{34}$, J.-F. Grivaz ${ }^{117}$, J.P. Grohs ${ }^{44}$, A. Grohsjean ${ }^{42}$, E. Gross ${ }^{172}$, J. Grosse-Knetter ${ }^{54}$,

G.C. Grossi ${ }^{79}$, Z.J. Grout ${ }^{149}$, L. Guan ${ }^{89}$, J. Guenther ${ }^{128}$, F. Guescini ${ }^{49}$, D. Guest ${ }^{163}$, O. Gueta ${ }^{153}$,

E. Guido ${ }^{50 a, 50 b}$, T. Guillemin ${ }^{117}$, S. Guindon ${ }^{2}$, U. Gul ${ }^{53}$, C. Gumpert ${ }^{30}$, J. Guo ${ }^{33 e}$, Y. Guo ${ }^{33 b, o}$,

S. Gupta ${ }^{120}$, G. Gustavino ${ }^{132 a, 132 b}$, P. Gutierrez ${ }^{113}$, N.G. Gutierrez Ortiz ${ }^{78}$, C. Gutschow ${ }^{44}$,

C. Guyot ${ }^{136}$, C. Gwenlan ${ }^{120}$, C.B. Gwilliam ${ }^{74}$, A. Haas $^{110}$, C. Haber ${ }^{15}$, H.K. Hadavand ${ }^{8}$,

N. Haddad ${ }^{135 e}$, P. Haefner ${ }^{21}$, S. Hageböck ${ }^{21}$, Z. Hajduk ${ }^{39}$, H. Hakobyan ${ }^{177}$, M. Haleem ${ }^{42}$,

J. Haley ${ }^{114}$, D. Hall ${ }^{120}$, G. Halladjian ${ }^{90}$, G.D. Hallewell ${ }^{85}$, K. Hamacher ${ }^{175}$, P. Hamal ${ }^{115}$,

K. Hamano ${ }^{169}$, A. Hamilton ${ }^{145 a}$, G.N. Hamity ${ }^{139}$, P.G. Hamnett ${ }^{42}$, L. Han ${ }^{33 b}$, K. Hanagaki ${ }^{66, r}$,

K. Hanawa ${ }^{155}$, M. Hance ${ }^{137}$, B. Haney ${ }^{122}$, P. Hanke ${ }^{58 a}$, R. Hanna ${ }^{136}$, J.B. Hansen ${ }^{36}$,

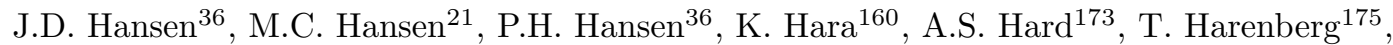

F. Hariri ${ }^{117}$, S. Harkusha ${ }^{92}$, R.D. Harrington ${ }^{46}$, P.F. Harrison ${ }^{170}$, F. Hartjes ${ }^{107}$, M. Hasegawa ${ }^{67}$,

Y. Hasegawa ${ }^{140}$, A. Hasib ${ }^{113}$, S. Hassani ${ }^{136}$, S. Haug ${ }^{17}$, R. Hauser ${ }^{90}$, L. Hauswald ${ }^{44}$,

M. Havranek ${ }^{127}$, C.M. Hawkes ${ }^{18}$, R.J. Hawkings ${ }^{30}$, A.D. Hawkins ${ }^{81}$, T. Hayashi ${ }^{160}$, D. Hayden ${ }^{90}$,

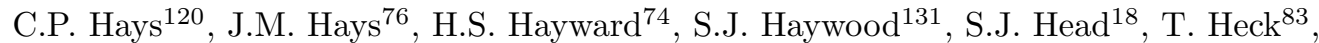

V. Hedberg ${ }^{81}$, L. Heelan ${ }^{8}$, S. Heim ${ }^{122}$, T. Heim ${ }^{175}$, B. Heinemann ${ }^{15}$, L. Heinrich ${ }^{110}$, J. Hejbal ${ }^{127}$,

L. Helary ${ }^{22}$, S. Hellman ${ }^{146 a, 146 b}$, C. Helsens ${ }^{30}$, J. Henderson ${ }^{120}$, R.C.W. Henderson ${ }^{72}$, Y. Heng ${ }^{173}$,

C. Hengler ${ }^{42}$, S. Henkelmann ${ }^{168}$, A. Henrichs ${ }^{176}$, A.M. Henriques Correia ${ }^{30}$, S. Henrot-Versille ${ }^{117}$,

G.H. Herbert ${ }^{16}$, Y. Hernández Jiménez ${ }^{167}$, G. Herten ${ }^{48}$, R. Hertenberger ${ }^{100}$, L. Hervas ${ }^{30}$,

G.G. Hesketh ${ }^{78}$, N.P. Hessey ${ }^{107}$, J.W. Hetherly ${ }^{40}$, R. Hickling ${ }^{76}$, E. Higón-Rodriguez ${ }^{167}$,

E. Hill ${ }^{169}$, J.C. Hill ${ }^{28}$, K.H. Hiller ${ }^{42}$, S.J. Hillier ${ }^{18}$, I. Hinchliffe ${ }^{15}$, E. Hines ${ }^{122}$, R.R. Hinman ${ }^{15}$,

M. Hirose ${ }^{157}$, D. Hirschbuehl ${ }^{175}$, J. Hobbs ${ }^{148}$, N. Hod ${ }^{107}$, M.C. Hodgkinson ${ }^{139}$, P. Hodgson ${ }^{139}$,

A. Hoecker ${ }^{30}$, M.R. Hoeferkamp ${ }^{105}$, F. Hoenig ${ }^{100}$, M. Hohlfeld ${ }^{83}$, D. Hohn ${ }^{21}$, T.R. Holmes ${ }^{15}$,

M. Homann ${ }^{43}$, T.M. Hong ${ }^{125}$, W.H. Hopkins ${ }^{116}$, Y. Horii ${ }^{103}$, A.J. Horton ${ }^{142}$, J-Y. Hostachy ${ }^{55}$, 
S. Hou ${ }^{151}$, A. Hoummada ${ }^{135 a}$, J. Howard ${ }^{120}$, J. Howarth ${ }^{42}$, M. Hrabovsky ${ }^{115}$, I. Hristova ${ }^{16}$, J. Hrivnac $^{117}$, T. Hryn'ova ${ }^{5}$, A. Hrynevich ${ }^{93}$, C. Hsu ${ }^{145 c}$, P.J. Hsu ${ }^{151, s}$, S.-C. Hsu ${ }^{138}$, D. Hu ${ }^{35}$, Q. Hu ${ }^{33 b}$, X. Hu ${ }^{89}$, Y. Huang ${ }^{42}$, Z. Hubacek ${ }^{128}$, F. Hubaut ${ }^{85}$, F. Huegging ${ }^{21}$, T.B. Huffman ${ }^{120}$, E.W. Hughes ${ }^{35}$, G. Hughes ${ }^{72}$, M. Huhtinen ${ }^{30}$, T.A. Hülsing ${ }^{83}$, N. Huseynov ${ }^{65, b}$, J. Huston ${ }^{90}$, J. Huth $^{57}$, G. Iacobucci ${ }^{49}$, G. Iakovidis ${ }^{25}$, I. Ibragimov ${ }^{141}$, L. Iconomidou-Fayard ${ }^{117}$, E. Ideal ${ }^{176}$, Z. Idrissi ${ }^{135 e}$, P. Iengo ${ }^{30}$, O. Igonkina ${ }^{107}$, T. Iizawa ${ }^{171}$, Y. Ikegami ${ }^{66}$, K. Ikematsu ${ }^{141}$, M. Ikeno ${ }^{66}$, Y. Ilchenko ${ }^{31, t}$, D. Iliadis ${ }^{154}$, N. Ilic ${ }^{143}$, T. Ince ${ }^{101}$, G. Introzzi ${ }^{121 a, 121 b}$, P. Ioannou ${ }^{9}$, M. Iodice $^{134 a}$, K. Iordanidou ${ }^{35}$, V. Ippolito ${ }^{57}$, A. Irles Quiles ${ }^{167}$, C. Isaksson ${ }^{166}$, M. Ishino ${ }^{68}$, M. Ishitsuka ${ }^{157}$, R. Ishmukhametov ${ }^{111}$, C. Issever ${ }^{120}$, S. Istin ${ }^{19 a}$, J.M. Iturbe Ponce ${ }^{84}$, R. Iuppa ${ }^{133 a, 133 b}$, J. Ivarsson $^{81}$, W. Iwanski ${ }^{39}$, H. Iwasaki ${ }^{66}$, J.M. Izen ${ }^{41}$, V. Izzo ${ }^{104 a}$, S. Jabbar ${ }^{3}$, B. Jackson ${ }^{122}$, M. Jackson ${ }^{74}$, P. Jackson ${ }^{1}$, M.R. Jaekel ${ }^{30}$, V. Jain ${ }^{2}$, K. Jakobs ${ }^{48}$, S. Jakobsen ${ }^{30}$, T. Jakoubek ${ }^{127}$, J. Jakubek ${ }^{128}$, D.O. Jamin ${ }^{114}$, D.K. Jana ${ }^{79}$, E. Jansen ${ }^{78}$, R. Jansky ${ }^{62}$, J. Janssen ${ }^{21}$, M. Janus ${ }^{54}$, G. Jarlskog ${ }^{81}$, N. Javadov ${ }^{65, b}$, T. Javưrek ${ }^{48}$, L. Jeanty ${ }^{15}$, J. Jejelava ${ }^{51 a, u}$, G.-Y. Jeng ${ }^{150}$, D. Jennens ${ }^{88}$, P. Jenni ${ }^{48, v}$, J. Jentzsch ${ }^{43}$, C. Jeske ${ }^{170}$, S. Jézéquel ${ }^{5}$, H. Ji ${ }^{173}$, J. Jia ${ }^{148}$, Y. Jiang ${ }^{33 b}$, S. Jiggins ${ }^{78}$, J. Jimenez Pena ${ }^{167}$, S. Jin ${ }^{33 a}$, A. Jinaru ${ }^{26 b}$, O. Jinnouchi ${ }^{157}$, M.D. Joergensen ${ }^{36}$, P. Johansson ${ }^{139}$, K.A. Johns ${ }^{7}$, W.J. Johnson ${ }^{138}$, K. Jon-And ${ }^{146 a, 146 b}$, G. Jones ${ }^{170}$, R.W.L. Jones ${ }^{72}$, T.J. Jones ${ }^{74}$, J. Jongmanns ${ }^{58 a}$, P.M. Jorge ${ }^{126 a, 126 b}$, K.D. Joshi ${ }^{84}$, J. Jovicevic ${ }^{159 a}$, X. Ju ${ }^{173}$, A. Juste Rozas ${ }^{12, q}$, M. Kaci ${ }^{167}$, A. Kaczmarska ${ }^{39}$, M. Kado ${ }^{117}$, H. Kagan ${ }^{111}$, M. Kagan ${ }^{143}$, S.J. Kahn ${ }^{85}$, E. Kajomovitz ${ }^{45}$, C.W. Kalderon ${ }^{120}$, S. Kama ${ }^{40}$, A. Kamenshchikov ${ }^{130}$, N. Kanaya ${ }^{155}$, S. Kaneti ${ }^{28}$, V.A. Kantserov ${ }^{98}$, J. Kanzaki ${ }^{66}$, B. Kaplan ${ }^{110}$, L.S. Kaplan ${ }^{173}$, A. Kapliy ${ }^{31}$, D. Kar ${ }^{145 c}$, K. Karakostas ${ }^{10}$, A. Karamaoun ${ }^{3}$, N. Karastathis ${ }^{10,107}$, M.J. Kareem ${ }^{54}$, E. Karentzos ${ }^{10}$, M. Karnevskiy ${ }^{83}$, S.N. Karpov ${ }^{65}$, Z.M. Karpova ${ }^{65}$, K. Karthik ${ }^{110}$, V. Kartvelishvili ${ }^{72}$, A.N. Karyukhin ${ }^{130}$, K. Kasahara ${ }^{160}$, L. Kashif ${ }^{173}$, R.D. Kass ${ }^{111}$,

A. Kastanas ${ }^{14}$, Y. Kataoka ${ }^{155}$, C. Kato ${ }^{155}$, A. Katre ${ }^{49}$, J. Katzy ${ }^{42}$, K. Kawade ${ }^{103}$, K. Kawagoe ${ }^{70}$, T. Kawamoto ${ }^{155}$, G. Kawamura ${ }^{54}$, S. Kazama ${ }^{155}$, V.F. Kazanin ${ }^{109, c}$, R. Keeler ${ }^{169}$, R. Kehoe ${ }^{40}$, J.S. Keller ${ }^{42}$, J.J. Kempster ${ }^{77}$, H. Keoshkerian ${ }^{84}$, O. Kepka ${ }^{127}$, B.P. Kerševan ${ }^{75}$, S. Kersten ${ }^{175}$, R.A. Keyes ${ }^{87}$, F. Khalil-zada ${ }^{11}$, H. Khandanyan ${ }^{146 a, 146 b}$, A. Khanov ${ }^{114}$, A.G. Kharlamov ${ }^{109, c}$, T.J. Khoo ${ }^{28}$, V. Khovanskiy ${ }^{97}$, E. Khramov ${ }^{65}$, J. Khubua ${ }^{51 b, w}$, S. Kido ${ }^{67}$, H.Y. Kim ${ }^{8}$, S.H. Kim ${ }^{160}$, Y.K. Kim ${ }^{31}$, N. Kimura ${ }^{154}$, O.M. Kind ${ }^{16}$, B.T. King ${ }^{74}$, M. King ${ }^{167}$, S.B. King ${ }^{168}$, J. Kirk ${ }^{131}$, A.E. Kiryunin ${ }^{101}$, T. Kishimoto ${ }^{67}$, D. Kisielewska ${ }^{38 a}$, F. Kiss ${ }^{48}$, K. Kiuchi ${ }^{160}$, O. Kivernyk ${ }^{136}$, E. Kladiva ${ }^{144 b}$, M.H. Klein ${ }^{35}$, M. Klein ${ }^{74}$, U. Klein ${ }^{74}$, K. Kleinknecht ${ }^{83}$, P. Klimek ${ }^{146 a, 146 b}$, A. Klimentov ${ }^{25}$, R. Klingenberg ${ }^{43}$, J.A. Klinger ${ }^{139}$, T. Klioutchnikova ${ }^{30}$, E.-E. Kluge ${ }^{58 a}$, P. Kluit ${ }^{107}$, S. Kluth ${ }^{101}$, J. Knapik ${ }^{39}$, E. Kneringer ${ }^{62}$, E.B.F.G. Knoops ${ }^{85}$, A. Knue ${ }^{53}$, A. Kobayashi ${ }^{155}$, D. Kobayashi ${ }^{157}$, T. Kobayashi ${ }^{155}$, M. Kobel ${ }^{44}$, M. Kocian ${ }^{143}$, P. Kodys ${ }^{129}$, T. Koffas ${ }^{29}$, E. Koffeman ${ }^{107}$, L.A. Kogan ${ }^{120}$, S. Kohlmann ${ }^{175}$, Z. Kohout ${ }^{128}$, T. Kohriki ${ }^{66}$, T. Koi ${ }^{143}$, H. Kolanoski ${ }^{16}$, M. Kolb ${ }^{58 b}$, I. Koletsou ${ }^{5}$, A.A. Komar ${ }^{96, *}$, Y. Komori ${ }^{155}$, T. Kondo ${ }^{66}$, N. Kondrashova ${ }^{42}$, K. Köneke ${ }^{48}$, A.C. König ${ }^{106}$, T. Kono ${ }^{66}$, R. Konoplich ${ }^{110, x}$, N. Konstantinidis ${ }^{78}$, R. Kopeliansky ${ }^{152}$, S. Koperny ${ }^{38 a}$, L. Köpke ${ }^{83}$, A.K. Kopp ${ }^{48}$, K. Korcyl ${ }^{39}$, K. Kordas ${ }^{154}$, A. Korn ${ }^{78}$, A.A. Korol ${ }^{109, c}$, I. Korolkov ${ }^{12}$, E.V. Korolkova ${ }^{139}$, O. Kortner ${ }^{101}$, S. Kortner ${ }^{101}$, T. Kosek ${ }^{129}$, V.V. Kostyukhin ${ }^{21}$, V.M. Kotov ${ }^{65}$, A. Kotwal ${ }^{45}$,

A. Kourkoumeli-Charalampidi ${ }^{154}$, C. Kourkoumelis ${ }^{9}$, V. Kouskoura ${ }^{25}$, A. Koutsman ${ }^{159 a}$, R. Kowalewski ${ }^{169}$, T.Z. Kowalski ${ }^{38 a}$, W. Kozanecki ${ }^{136}$, A.S. Kozhin ${ }^{130}$, V.A. Kramarenko ${ }^{99}$, G. Kramberger ${ }^{75}$, D. Krasnopevtsev ${ }^{98}$, M.W. Krasny ${ }^{80}$, A. Krasznahorkay ${ }^{30}$, J.K. Kraus ${ }^{21}$, A. Kravchenko ${ }^{25}$, S. Kreiss ${ }^{110}$, M. Kretz ${ }^{58 c}$, J. Kretzschmar ${ }^{74}$, K. Kreutzfeldt ${ }^{52}$, P. Krieger ${ }^{158}$, K. Krizka ${ }^{31}$, K. Kroeninger ${ }^{43}$, H. Kroha ${ }^{101}$, J. Kroll ${ }^{122}$, J. Kroseberg ${ }^{21}$, J. Krstic ${ }^{13}$, U. Kruchonak ${ }^{65}$, H. Krüger ${ }^{21}$, N. Krumnack ${ }^{64}$, A. Kruse ${ }^{173}$, M.C. Kruse ${ }^{45}$, M. Kruskal ${ }^{22}$, T. Kubota ${ }^{88}$, H. Kucuk ${ }^{78}$, S. Kuday ${ }^{4 b}$, S. Kuehn ${ }^{48}$, A. Kugel ${ }^{58 c}$, F. Kuger ${ }^{174}$, A. Kuhl ${ }^{137}$, T. Kuhl ${ }^{42}$, V. Kukhtin ${ }^{65}$, R. Kukla ${ }^{136}$, Y. Kulchitsky ${ }^{92}$, S. Kuleshov ${ }^{32 b}$, M. Kuna ${ }^{132 a, 132 b}$, 
T. Kunigo ${ }^{68}$, A. Kupco ${ }^{127}$, H. Kurashige ${ }^{67}$, Y.A. Kurochkin ${ }^{92}$, V. Kus ${ }^{127}$, E.S. Kuwertz ${ }^{169}$, M. Kuze ${ }^{157}$, J. Kvita ${ }^{115}$, T. Kwan ${ }^{169}$, D. Kyriazopoulos ${ }^{139}$, A. La Rosa ${ }^{137}$, J.L. La Rosa Navarro ${ }^{24 d}$, L. La Rotonda ${ }^{37 a, 37 b}$, C. Lacasta ${ }^{167}$, F. Lacava ${ }^{132 a, 132 b}$, J. Lacey ${ }^{29}$, H. Lacker ${ }^{16}$, D. Lacour ${ }^{80}$, V.R. Lacuesta ${ }^{167}$, E. Ladygin ${ }^{65}$, R. Lafaye ${ }^{5}$, B. Laforge ${ }^{80}$,

T. Lagouri ${ }^{176}$, S. Lai ${ }^{54}$, L. Lambourne ${ }^{78}$, S. Lammers ${ }^{61}$, C.L. Lampen ${ }^{7}$, W. Lampl ${ }^{7}$, E. Lançon ${ }^{136}$, U. Landgraf ${ }^{48}$, M.P.J. Landon ${ }^{76}$, V.S. Lang ${ }^{58 a}$, J.C. Lange ${ }^{12}$, A.J. Lankford ${ }^{163}$, F. Lanni ${ }^{25}$,

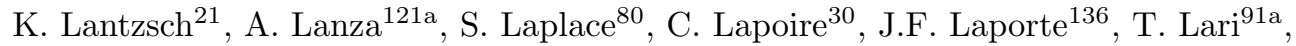
F. Lasagni Manghi ${ }^{20 a, 20 b}$, M. Lassnig ${ }^{30}$, P. Laurelli ${ }^{47}$, W. Lavrijsen ${ }^{15}$, A.T. Law ${ }^{137}$, P. Laycock ${ }^{74}$, T. Lazovich ${ }^{57}$, O. Le Dortz ${ }^{80}$, E. Le Guirriec ${ }^{85}$, E. Le Menedeu ${ }^{12}$, M. LeBlanc ${ }^{169}$, T. LeCompte ${ }^{6}$, F. Ledroit-Guillon ${ }^{55}$, C.A. Lee ${ }^{145 a}$, S.C. Lee ${ }^{151}$, L. Lee ${ }^{1}$, G. Lefebvre ${ }^{80}$, M. Lefebvre ${ }^{169}$, F. Legger ${ }^{100}$, C. Leggett ${ }^{15}$, A. Lehan ${ }^{74}$, G. Lehmann Miotto ${ }^{30}$, X. Lei ${ }^{7}$, W.A. Leight ${ }^{29}$, A. Leisos ${ }^{154, y}$, A.G. Leister ${ }^{176}$, M.A.L. Leite ${ }^{24 d}$, R. Leitner ${ }^{129}$, D. Lellouch ${ }^{172}$, B. Lemmer ${ }^{54}$, K.J.C. Leney ${ }^{78}$, T. Lenz ${ }^{21}$, B. Lenzi ${ }^{30}$, R. Leone ${ }^{7}$, S. Leone ${ }^{124 a, 124 b}$, C. Leonidopoulos ${ }^{46}$, S. Leontsinis ${ }^{10}$, C. Leroy ${ }^{95}$, C.G. Lester ${ }^{28}$, M. Levchenko ${ }^{123}$, J. Levêque ${ }^{5}$, D. Levin ${ }^{89}$, L.J. Levinson ${ }^{172}$, M. Levy ${ }^{18}$, A. Lewis ${ }^{120}$, A.M. Leyko ${ }^{21}$, M. Leyton ${ }^{41}$, B. Li ${ }^{33 b}, z, \mathrm{H}^{3} \mathrm{Li}^{148}$,

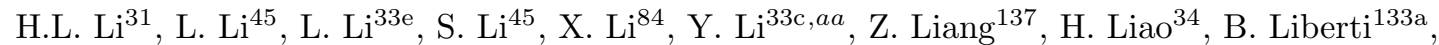

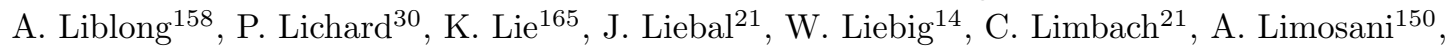
S.C. $\operatorname{Lin}^{151, a b}$, T.H. $\operatorname{Lin}^{83}$, F. Linde ${ }^{107}$, B.E. Lindquist ${ }^{148}$, J.T. Linnemann ${ }^{90}$, E. Lipeles ${ }^{122}$, A. Lipniacka ${ }^{14}$, M. Lisovyi ${ }^{58 b}$, T.M. Liss ${ }^{165}$, D. Lissauer ${ }^{25}$, A. Lister ${ }^{168}$, A.M. Litke ${ }^{137}$, B. $\mathrm{Liu}^{151, a c}$, D. $\mathrm{Liu}^{151}$, H. Liu ${ }^{89}$, J. Liu ${ }^{85}$, J.B. Liu ${ }^{33 b}$, K. Liu ${ }^{85}$, L. Liu ${ }^{165}$, M. Liu ${ }^{45}$, M. Liu ${ }^{33 b}$, Y. Liu ${ }^{33 b}$, M. Livan ${ }^{121 a, 121 b}$, A. Lleres ${ }^{55}$, J. Llorente Merino ${ }^{82}$, S.L. Lloyd ${ }^{76}$, F. Lo Sterzo ${ }^{151}$, E. Lobodzinska ${ }^{42}$, P. Loch $^{7}$, W.S. Lockman ${ }^{137}$, F.K. Loebinger ${ }^{84}$, A.E. Loevschall-Jensen ${ }^{36}$, K.M. Loew ${ }^{23}$, A. Loginov ${ }^{176}$, T. Lohse ${ }^{16}$, K. Lohwasser ${ }^{42}$, M. Lokajicek ${ }^{127}$, B.A. Long ${ }^{22}$, J.D. Long ${ }^{165}$, R.E. Long ${ }^{72}$, K.A. Looper ${ }^{111}$, L. Lopes ${ }^{126 a}$, D. Lopez Mateos ${ }^{57}$, B. Lopez Paredes ${ }^{139}$, I. Lopez Paz ${ }^{12}$, J. Lorenz ${ }^{100}$, N. Lorenzo Martinez ${ }^{61}$, M. Losada ${ }^{162}$,

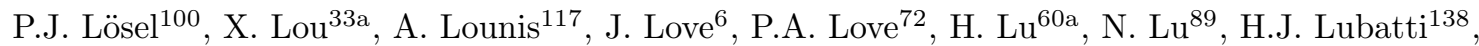
C. Luci ${ }^{132 a, 132 b}$, A. Lucotte ${ }^{55}$, C. Luedtke ${ }^{48}$, F. Luehring ${ }^{61}$, W. Lukas ${ }^{62}$, L. Luminari ${ }^{132 a}$, O. Lundberg ${ }^{146 a, 146 b}$, B. Lund-Jensen ${ }^{147}$, D. Lynn ${ }^{25}$, R. Lysak ${ }^{127}$, E. Lytken ${ }^{81}, \mathrm{H} \mathrm{Ma}^{25}$, L.L. Ma ${ }^{33 d}$, G. Maccarrone ${ }^{47}$, A. Macchiolo ${ }^{101}$, C.M. Macdonald ${ }^{139}$, B. Maček ${ }^{75}$, J. Machado Miguens ${ }^{122,126 b}$, D. Macina ${ }^{30}$, D. Madaffari ${ }^{85}$, R. Madar ${ }^{34}$, H.J. Maddocks ${ }^{72}$, W.F. Mader ${ }^{44}$, A. Madsen ${ }^{166}$, J. Maeda ${ }^{67}$, S. Maeland ${ }^{14}$, T. Maeno ${ }^{25}$, A. Maevskiy ${ }^{99}$,

E. Magradze ${ }^{54}$, K. Mahboubi ${ }^{48}$, J. Mahlstedt ${ }^{107}$, C. Maiani ${ }^{136}$, C. Maidantchik ${ }^{24 a}$, A.A. Maier ${ }^{101}$, T. Maier ${ }^{100}$, A. Maio ${ }^{126 a, 126 b, 126 d}$, S. Majewski ${ }^{116}$, Y. Makida ${ }^{66}$, N. Makovec ${ }^{117}$, B. Malaescu ${ }^{80}$, Pa. Malecki ${ }^{39}$, V.P. Maleev ${ }^{123}$, F. Malek ${ }^{55}$, U. Mallik ${ }^{63}$, D. Malon ${ }^{6}$, C. Malone ${ }^{143}$, S. Maltezos ${ }^{10}$, V.M. Malyshev ${ }^{109}$, S. Malyukov ${ }^{30}$, J. Mamuzic ${ }^{42}$, G. Mancini ${ }^{47}$, B. Mandelli ${ }^{30}$, L. Mandelli ${ }^{91 a}$, I. Mandic ${ }^{75}$, R. Mandrysch ${ }^{63}$, J. Maneira ${ }^{126 a, 126 b}$, L. Manhaes de Andrade Filho ${ }^{24 b}$, J. Manjarres Ramos ${ }^{159 b}$, A. Mann ${ }^{100}$, A. Manousakis-Katsikakis ${ }^{9}$, B. Mansoulie ${ }^{136}$, R. Mantifel ${ }^{87}$, M. Mantoani ${ }^{54}$, L. Mapelli ${ }^{30}$, L. March ${ }^{145 c}$, G. Marchiori ${ }^{80}$, M. Marcisovsky ${ }^{127}$, C.P. Marino ${ }^{169}$, M. Marjanovic ${ }^{13}$, D.E. Marley ${ }^{89}$, F. Marroquim ${ }^{24 a}$, S.P. Marsden ${ }^{84}$, Z. Marshall ${ }^{15}$, L.F. Marti ${ }^{17}$, S. Marti-Garcia ${ }^{167}$, B. Martin ${ }^{90}$, T.A. Martin ${ }^{170}$, V.J. Martin ${ }^{46}$, B. Martin dit Latour ${ }^{14}$, M. Martinez ${ }^{12, q}$, S. Martin-Haugh ${ }^{131}$, V.S. Martoiu ${ }^{26 b}$, A.C. Martyniuk ${ }^{78}$, M. Marx ${ }^{138}$, F. Marzano ${ }^{132 a}$, A. Marzin ${ }^{30}$, L. Masetti ${ }^{83}$, T. Mashimo ${ }^{155}$, R. Mashinistov ${ }^{96}$, J. Masik $^{84}$, A.L. Maslennikov ${ }^{109, c}$, I. Massa ${ }^{20 a, 20 b}$, L. Massa ${ }^{20 a, 20 b}$, P. Mastrandrea ${ }^{5}$,

A. Mastroberardino ${ }^{37 a, 37 b}$, T. Masubuchi ${ }^{155}$, P. Mättig ${ }^{175}$, J. Mattmann ${ }^{83}$, J. Maurer ${ }^{26 b}$, S.J. Maxfield ${ }^{74}$, D.A. Maximov ${ }^{109, c}$, R. Mazini ${ }^{151}$, S.M. Mazza ${ }^{91 a, 91 b}$, G. Mc Goldrick ${ }^{158}$, S.P. Mc Kee ${ }^{89}$, A. McCarn ${ }^{89}$, R.L. McCarthy ${ }^{148}$, T.G. McCarthy ${ }^{29}$, N.A. McCubbin ${ }^{131}$, K.W. McFarlane ${ }^{56, *}$, J.A. Mcfayden ${ }^{78}$, G. Mchedlidze ${ }^{54}$, S.J. McMahon ${ }^{131}$, R.A. McPherson ${ }^{169, l}$, M. Medinnis ${ }^{42}$, S. Meehan ${ }^{138}$, S. Mehlhase ${ }^{100}$, A. Mehta ${ }^{74}$, K. Meier ${ }^{58 a}$, C. Meineck ${ }^{100}$, 
B. Meirose ${ }^{41}$, B.R. Mellado Garcia ${ }^{145 c}$, F. Meloni ${ }^{17}$, A. Mengarelli20a,20b ${ }^{2}$ S. Menke ${ }^{101}$, E. Meoni ${ }^{161}$, K.M. Mercurio ${ }^{57}$, S. Mergelmeyer ${ }^{21}$, P. Mermod ${ }^{49}$, L. Merola $^{104 a, 104 b}$, C. Meroni ${ }^{91 a}$, F.S. Merritt ${ }^{31}$, A. Messina ${ }^{132 a, 132 b}$, J. Metcalfe ${ }^{25}$, A.S. Mete ${ }^{163}$, C. Meyer ${ }^{83}$, C. Meyer ${ }^{122}$, J-P. Meyer ${ }^{136}$, J. Meyer ${ }^{107}$, H. Meyer Zu Theenhausen ${ }^{58 a}$, R.P. Middleton ${ }^{131}$, S. Miglioranzi ${ }^{164 a, 164 c}$, L. Mijović ${ }^{21}$, G. Mikenberg ${ }^{172}$, M. Mikestikova ${ }^{127}$, M. Mikuž ${ }^{75}$, M. Milesi ${ }^{88}$, A. Milic ${ }^{30}$, D.W. Miller ${ }^{31}$, C. Mills ${ }^{46}$, A. Milov ${ }^{172}$, D.A. Milstead ${ }^{146 a, 146 b}$, A.A. Minaenko ${ }^{130}$, Y. Minami ${ }^{155}$, I.A. Minashvili ${ }^{65}$, A.I. Mincer ${ }^{110}$, B. Mindur ${ }^{38 a}$, M. Mineev ${ }^{65}$, Y. Ming ${ }^{173}$, L.M. Mir ${ }^{12}$, K.P. Mistry ${ }^{122}$, T. Mitani ${ }^{171}$, J. Mitrevski ${ }^{100}$, V.A. Mitsou ${ }^{167}$, A. Miucci ${ }^{49}$, P.S. Miyagawa ${ }^{139}$, J.U. Mjörnmark ${ }^{81}$, T. Moa ${ }^{146 a, 146 b}$, K. Mochizuki $^{85}$, S. Mohapatra ${ }^{35}$, W. Mohr ${ }^{48}$, S. Molander ${ }^{146 a, 146 b}$, R. Moles-Valls ${ }^{21}$, R. Monden ${ }^{68}$, K. Mönig ${ }^{42}$, C. Monini ${ }^{55}$, J. Monk ${ }^{36}$, E. Monnier ${ }^{85}$, A. Montalbano ${ }^{148}$, J. Montejo Berlingen ${ }^{12}$, F. Monticelli ${ }^{71}$, S. Monzani ${ }^{132 a, 132 b}$, R.W. Moore ${ }^{3}$, N. Morange ${ }^{117}$, D. Moreno ${ }^{162}$, M. Moreno Llácer ${ }^{54}$, P. Morettini ${ }^{50 a}$, D. Mori ${ }^{142}$, T. Mori ${ }^{155}$, M. Morii ${ }^{57}$, M. Morinaga ${ }^{155}$, V. Morisbak ${ }^{119}$, S. Moritz $^{83}$, A.K. Morley ${ }^{150}$, G. Mornacchi ${ }^{30}$, J.D. Morris ${ }^{76}$, S.S. Mortensen ${ }^{36}$, A. Morton ${ }^{53}$, L. Morvaj ${ }^{103}$, M. Mosidze ${ }^{51 b}$, J. Moss ${ }^{143}$, K. Motohashi ${ }^{157}$, R. Mount ${ }^{143}$, E. Mountricha ${ }^{25}$, S.V. Mouraviev ${ }^{96, *}$, E.J.W. Moyse ${ }^{86}$, S. Muanza ${ }^{85}$, R.D. Mudd ${ }^{18}$, F. Mueller ${ }^{101}$, J. Mueller ${ }^{125}$, R.S.P. Mueller ${ }^{100}$, T. Mueller ${ }^{28}$, D. Muenstermann ${ }^{49}$, P. Mullen ${ }^{53}$, G.A. Mullier ${ }^{17}$, F.J. Munoz Sanchez ${ }^{84}$, J.A. Murillo Quijada ${ }^{18}$, W.J. Murray ${ }^{170,131}$, H. Musheghyan ${ }^{54}$, E. Musto ${ }^{152}$, A.G. Myagkov ${ }^{130, a d}$, M. Myska ${ }^{128}$, B.P. Nachman ${ }^{143}$, O. Nackenhorst ${ }^{54}$, J. Nadal ${ }^{54}$, K. Nagai ${ }^{120}$, R. Nagai ${ }^{157}$,

Y. Nagai ${ }^{85}$, K. Nagano ${ }^{66}$, A. Nagarkar ${ }^{111}$, Y. Nagasaka ${ }^{59}$, K. Nagata ${ }^{160}$, M. Nagel ${ }^{101}$, E. Nagy ${ }^{85}$, A.M. Nairz ${ }^{30}$, Y. Nakahama ${ }^{30}$, K. Nakamura ${ }^{66}$, T. Nakamura ${ }^{155}$, I. Nakano ${ }^{112}$,

H. Namasivayam ${ }^{41}$, R.F. Naranjo Garcia ${ }^{42}$, R. Narayan $^{31}$, D.I. Narrias Villar ${ }^{58 a}$, T. Naumann ${ }^{42}$, G. Navarro ${ }^{162}$, R. Nayyar ${ }^{7}$, H.A. Neal ${ }^{89}$, P.Yu. Nechaeva ${ }^{96}$, T.J. Neep ${ }^{84}$, P.D. Nef ${ }^{143}$, A. Negri ${ }^{121 a, 121 b}$, M. Negrini ${ }^{20 a}$, S. Nektarijevic ${ }^{106}$, C. Nellist ${ }^{117}$, A. Nelson ${ }^{163}$, S. Nemecek ${ }^{127}$, P. Nemethy ${ }^{110}$, A.A. Nepomuceno ${ }^{24 a}$, M. Nessi ${ }^{30, a e}$, M.S. Neubauer ${ }^{165}$, M. Neumann ${ }^{175}$, R.M. Neves ${ }^{110}$, P. Nevski ${ }^{25}$, P.R. Newman ${ }^{18}$, D.H. Nguyen ${ }^{6}$, R.B. Nickerson ${ }^{120}$, R. Nicolaidou ${ }^{136}$, B. Nicquevert ${ }^{30}$, J. Nielsen ${ }^{137}$, N. Nikiforou ${ }^{35}$, A. Nikiforov ${ }^{16}$, V. Nikolaenko ${ }^{130, a d}$, I. Nikolic-Audit ${ }^{80}$, K. Nikolopoulos ${ }^{18}$, J.K. Nilsen ${ }^{119}$, P. Nilsson ${ }^{25}$, Y. Ninomiya ${ }^{155}$, A. Nisati ${ }^{132 a}$, R. Nisius ${ }^{101}$, T. Nobe ${ }^{155}$, M. Nomachi ${ }^{118}$, I. Nomidis ${ }^{29}$, T. Nooney ${ }^{76}$, S. Norberg ${ }^{113}$, M. Nordberg ${ }^{30}$, O. Novgorodova ${ }^{44}$, S. Nowak ${ }^{101}$, M. Nozaki ${ }^{66}$, L. Nozka ${ }^{115}$, K. Ntekas ${ }^{10}$, G. Nunes Hanninger ${ }^{88}$, T. Nunnemann ${ }^{100}$, E. Nurse ${ }^{78}$, F. Nuti ${ }^{88}$, F. O'grady ${ }^{7}$, D.C. O’Neil ${ }^{142}$, V. O'Shea ${ }^{53}$, F.G. Oakham ${ }^{29, d}$, H. Oberlack ${ }^{101}$, T. Obermann ${ }^{21}$, J. Ocariz ${ }^{80}$, A. Ochi ${ }^{67}$,

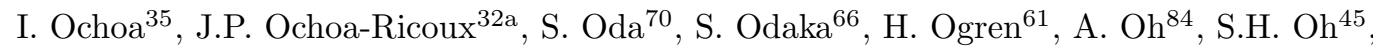
C.C. $\mathrm{Ohm}^{15}$, H. Ohman ${ }^{166}$, H. Oide ${ }^{30}$, W. Okamura ${ }^{118}$, H. Okawa ${ }^{160}$, Y. Okumura ${ }^{31}$,

T. Okuyama ${ }^{66}$, A. Olariu ${ }^{26 b}$, S.A. Olivares Pino ${ }^{46}$, D. Oliveira Damazio ${ }^{25}$, A. Olszewski ${ }^{39}$, J. Olszowska ${ }^{39}$, A. Onofre ${ }^{126 a, 126 e}$, K. Onogi ${ }^{103}$, P.U.E. Onyisi ${ }^{31, t}$, C.J. Oram ${ }^{159 a}$, M.J. Oreglia ${ }^{31}$, Y. Oren ${ }^{153}$, D. Orestano ${ }^{134 a, 134 b}$, N. Orlando ${ }^{154}$, C. Oropeza Barrera ${ }^{53}$, R.S. Orr ${ }^{158}$, B. Osculati ${ }^{50 a, 50 b}$, R. Ospanov ${ }^{84}$, G. Otero y Garzon ${ }^{27}$, H. Otono ${ }^{70}$, M. Ouchrif ${ }^{135 d}$, F. Ould-Saada ${ }^{119}$, A. Ouraou ${ }^{136}$, K.P. Oussoren ${ }^{107}$, Q. Ouyang ${ }^{33 a}$, A. Ovcharova ${ }^{15}$, M. Owen ${ }^{53}$, R.E. Owen ${ }^{18}$, V.E. Ozcan ${ }^{19 a}$, N. Ozturk ${ }^{8}$, K. Pachal ${ }^{142}$, A. Pacheco Pages ${ }^{12}$, C. Padilla Aranda ${ }^{12}$, M. Pagáčová ${ }^{48}$, S. Pagan Griso ${ }^{15}$, E. Paganis ${ }^{139}$, F. Paige ${ }^{25}$, P. Pais ${ }^{86}$, K. Pajchel ${ }^{119}$, G. Palacino ${ }^{159 b}$, S. Palestini ${ }^{30}$, M. Palka ${ }^{38 b}$, D. Pallin ${ }^{34}$, A. Palma ${ }^{126 a, 126 b}$, Y.B. Pan ${ }^{173}$, E.St. Panagiotopoulou ${ }^{10}$, C.E. Pandini ${ }^{80}$, J.G. Panduro Vazquez ${ }^{77}$, P. Pani ${ }^{146 a, 146 b}$, S. Panitkin ${ }^{25}$, D. Pantea ${ }^{26 b}$, L. Paolozzi ${ }^{49}$, Th.D. Papadopoulou ${ }^{10}$, K. Papageorgiou ${ }^{154}$, A. Paramonov ${ }^{6}$, D. Paredes Hernandez ${ }^{154}$, M.A. Parker ${ }^{28}$, K.A. Parker ${ }^{139}$, F. Parodi ${ }^{50 a, 50 b}$, J.A. Parsons ${ }^{35}$, U. Parzefall $^{48}$, E. Pasqualucci ${ }^{132 a}$, S. Passaggio ${ }^{50 a}$, F. Pastore ${ }^{134 a, 134 b, *}$, Fr. Pastore $^{77}$, G. Pásztor ${ }^{29}$, S. Pataraia ${ }^{175}$, N.D. Patel ${ }^{150}$, J.R. Pater ${ }^{84}$, T. Pauly ${ }^{30}$, J. Pearce ${ }^{169}$, B. Pearson ${ }^{113}$, L.E. Pedersen ${ }^{36}$, M. Pedersen ${ }^{119}$, S. Pedraza Lopez ${ }^{167}$, R. Pedro ${ }^{126 a, 126 b}$, S.V. Peleganchuk ${ }^{109, c}$, 
D. Pelikan ${ }^{166}$, O. Penc ${ }^{127}$, C. Peng ${ }^{33 a}$, H. Peng ${ }^{33 b}$, B. Penning ${ }^{31}$, J. Penwell ${ }^{61}$, D.V. Perepelitsa ${ }^{25}$, E. Perez Codina ${ }^{159 a}$, M.T. Pérez García-Estañ ${ }^{167}$, L. Perini ${ }^{11 a, 91 b}$, H. Pernegger ${ }^{30}$, S. Perrella ${ }^{104 a, 104 b}$, R. Peschke ${ }^{42}$, V.D. Peshekhonov ${ }^{65}$, K. Peters ${ }^{30}$, R.F.Y. Peters ${ }^{84}$, B.A. Petersen $^{30}$, T.C. Petersen ${ }^{36}$, E. Petit ${ }^{42}$, A. Petridis ${ }^{1}$, C. Petridou ${ }^{154}$, P. Petroff ${ }^{117}$, E. Petrolo ${ }^{132 a}$, F. Petrucci ${ }^{134 a, 134 b}$, N.E. Pettersson ${ }^{157}$, R. Pezoa ${ }^{32 b}$, P.W. Phillips ${ }^{131}$, G. Piacquadio ${ }^{143}$, E. Pianori ${ }^{170}$, A. Picazio ${ }^{49}$, E. Piccaro ${ }^{76}$, M. Piccinini2 ${ }^{20 a}, 20 \mathrm{~b}$, M.A. Pickering ${ }^{120}$, R. Piegaia ${ }^{27}$, D.T. Pignotti ${ }^{111}$, J.E. Pilcher ${ }^{31}$, A.D. Pilkington ${ }^{84}$, A.W.J. Pin $^{84}$, J. Pina ${ }^{126 a, 126 b, 126 d}$, M. Pinamonti164a,164c,af , J.L. Pinfold ${ }^{3}$, A. Pingel ${ }^{36}$, S. Pires ${ }^{80}$, H. Pirumov ${ }^{42}$, M. Pitt ${ }^{172}$, C. Pizio ${ }^{91 a, 91 b}$, L. Plazak ${ }^{144 a}$, M.-A. Pleier ${ }^{25}$, V. Pleskot ${ }^{129}$, E. Plotnikova ${ }^{65}$, P. Plucinski ${ }^{146 a, 146 b}$, D. Pluth ${ }^{64}$, R. Poettgen ${ }^{146 a, 146 b}$, L. Poggioli $^{117}$, D. Pohl ${ }^{21}$, G. Polesello ${ }^{121 a}$, A. Poley ${ }^{42}$, A. Policicchio ${ }^{37 a, 37 b}$, R. Polifka ${ }^{158}$, A. Polini ${ }^{20 a}$, C.S. Pollard ${ }^{53}$, V. Polychronakos ${ }^{25}$, K. Pommès ${ }^{30}$, L. Pontecorvo ${ }^{132 a}$, B.G. Pope ${ }^{90}$, G.A. Popeneciu ${ }^{26 c}$, D.S. Popovic ${ }^{13}$, A. Poppleton ${ }^{30}$, S. Pospisil ${ }^{128}$, K. Potamianos ${ }^{15}$, I.N. Potrap ${ }^{65}$, C.J. Potter ${ }^{149}$, C.T. Potter ${ }^{116}$, G. Poulard ${ }^{30}$, J. Poveda ${ }^{30}$, V. Pozdnyakov ${ }^{65}$, M.E. Pozo Astigarraga ${ }^{30}$, P. Pralavorio ${ }^{85}$, A. Pranko ${ }^{15}$, S. Prasad ${ }^{30}$, S. Prell ${ }^{64}$, D. Price ${ }^{84}$, L.E. Price ${ }^{6}$, M. Primavera ${ }^{73 a}$, S. Prince ${ }^{87}$, M. Proiss ${ }^{46}$, K. Prokofiev ${ }^{60 c}$, F. Prokoshin ${ }^{32 b}$, E. Protopapadaki ${ }^{136}$,

S. Protopopescu ${ }^{25}$, J. Proudfoot ${ }^{6}$, M. Przybycien ${ }^{38 a}$, E. Ptacek ${ }^{116}$, D. Puddu ${ }^{134 a, 134 b}$, E. Pueschel ${ }^{86}$, D. Puldon ${ }^{148}$, M. Purohit ${ }^{25, a g}$, P. Puzo ${ }^{117}$, J. Qian ${ }^{89}$, G. Qin ${ }^{53}$, Y. Qin ${ }^{84}$, A. Quadt $^{54}$, D.R. Quarrie ${ }^{15}$, W.B. Quayle ${ }^{164 a, 164 b}$, M. Queitsch-Maitland ${ }^{84}$, D. Quilty ${ }^{53}$, S. Raddum ${ }^{119}$, V. Radeka ${ }^{25}$, V. Radescu ${ }^{42}$, S.K. Radhakrishnan ${ }^{148}$, P. Radloff ${ }^{116}$, P. Rados $^{88}$, F. Ragusa ${ }^{91 a, 91 b}$, G. Rahal ${ }^{178}$, S. Rajagopalan ${ }^{25}$, M. Rammensee ${ }^{30}$, C. Rangel-Smith ${ }^{166}$, F. Rauscher ${ }^{100}$, S. Rave ${ }^{83}$, T. Ravenscroft ${ }^{53}$, M. Raymond ${ }^{30}$, A.L. Read ${ }^{119}$, N.P. Readioff ${ }^{74}$, D.M. Rebuzzi ${ }^{121 a, 121 b}$, A. Redelbach ${ }^{174}$, G. Redlinger ${ }^{25}$, R. Reece ${ }^{137}$, K. Reeves ${ }^{41}$, L. Rehnisch $^{16}$, J. Reichert ${ }^{122}$, H. Reisin ${ }^{27}$, C. Rembser ${ }^{30}$, H. Ren ${ }^{33 a}$, A. Renaud ${ }^{117}$, M. Rescigno ${ }^{132 a}$, S. Resconi ${ }^{91 a}$, O.L. Rezanova ${ }^{109, c}$, P. Reznicek ${ }^{129}$, R. Rezvani ${ }^{95}$, R. Richter ${ }^{101}$, S. Richter $^{78}$, E. Richter-Was ${ }^{38 b}$, O. Ricken ${ }^{21}$, M. Ridel ${ }^{80}$, P. Rieck ${ }^{16}$, C.J. Riegel ${ }^{175}$, J. Rieger ${ }^{54}$, O. Rifki ${ }^{113}$, M. Rijssenbeek ${ }^{148}$, A. Rimoldi ${ }^{121 a, 121 b}$, L. Rinaldi ${ }^{20 a}$, B. Ristić ${ }^{49}$, E. Ritsch ${ }^{30}$, I. Riu ${ }^{12}$, F. Rizatdinova ${ }^{114}$, E. Rizvi ${ }^{76}$, S.H. Robertson ${ }^{87, l}$, A. Robichaud-Veronneau ${ }^{87}$, D. Robinson ${ }^{28}$, J.E.M. Robinson ${ }^{42}$, A. Robson ${ }^{53}$, C. Roda ${ }^{124 a, 124 b}$, S. Roe ${ }^{30}$, O. Røhne ${ }^{119}$, A. Romaniouk ${ }^{98}$, M. Romano ${ }^{20 a, 20 b}$, S.M. Romano $\mathrm{Saez}^{34}$, E. Romero Adam ${ }^{167}$, N. Rompotis ${ }^{138}$, M. Ronzani ${ }^{48}$, L. $\operatorname{Roos}^{80}$, E. $\operatorname{Ros}^{167}$, S. Rosati ${ }^{132 a}$, K. Rosbach ${ }^{48}$, P. Rose ${ }^{137}$, P.L. Rosendahl ${ }^{14}$, O. Rosenthal ${ }^{141}$, V. Rossetti ${ }^{146 a, 146 b}$, E. Rossi ${ }^{104 a, 104 b}$, L.P. Rossi ${ }^{50 a}$, J.H.N. Rosten ${ }^{28}$, R. Rosten ${ }^{138}$, M. Rotaru ${ }^{26 b}$, I. Roth ${ }^{172}$, J. Rothberg ${ }^{138}$, D. Rousseau ${ }^{117}$, C.R. Royon ${ }^{136}$, A. Rozanov ${ }^{85}$, Y. Rozen ${ }^{152}$, X. Ruan ${ }^{145 c}$, F. Rubbo ${ }^{143}$, I. Rubinskiy ${ }^{42}$, V.I. Rud ${ }^{99}$, C. Rudolph ${ }^{44}$, M.S. Rudolph ${ }^{158}$, F. Rühr ${ }^{48}$, A. Ruiz-Martinez ${ }^{30}$, Z. Rurikova ${ }^{48}$, N.A. Rusakovich ${ }^{65}$, A. Ruschke ${ }^{100}$, H.L. Russell ${ }^{138}$, J.P. Rutherfoord ${ }^{7}$, N. Ruthmann ${ }^{30}$, Y.F. Ryabov ${ }^{123}$, M. Rybar ${ }^{165}$, G. Rybkin ${ }^{117}$, N.C. Ryder ${ }^{120}$, A.F. Saavedra ${ }^{150}$, G. Sabato ${ }^{107}$, S. Sacerdoti ${ }^{27}$, A. Saddique ${ }^{3}$, H.F-W. Sadrozinski ${ }^{137}$, R. Sadykov ${ }^{65}$, F. Safai Tehrani1 ${ }^{132 a}$, P. Saha ${ }^{108}$, M. Sahinsoy ${ }^{58 a}$, M. Saimpert ${ }^{136}$, T. Saito ${ }^{155}$, H. Sakamoto ${ }^{155}$, Y. Sakurai ${ }^{171}$, G. Salamanna ${ }^{134 a, 134 b}$, A. Salamon ${ }^{133 a}$, J.E. Salazar Loyola ${ }^{32 b}$, M. Saleem ${ }^{113}$, D. Salek ${ }^{107}$, P.H. Sales De Bruin ${ }^{138}$, D. Salihagic ${ }^{101}$, A. Salnikov ${ }^{143}$, J. Salt ${ }^{167}$, D. Salvatore ${ }^{37 a, 37 b}$, F. Salvatore ${ }^{149}$, A. Salvucci ${ }^{60 a}$, A. Salzburger $^{30}$, D. Sammel ${ }^{48}$, D. Sampsonidis ${ }^{154}$, A. Sanchez ${ }^{104 a, 104 b}$, J. Sánchez ${ }^{167}$, V. Sanchez Martinez ${ }^{167}$, H. Sandaker ${ }^{119}$, R.L. Sandbach ${ }^{76}$, H.G. Sander ${ }^{83}$, M.P. Sanders ${ }^{100}$, M. Sandhoff ${ }^{175}$, C. Sandoval ${ }^{162}$, R. Sandstroem ${ }^{101}$, D.P.C. Sankey ${ }^{131}$, M. Sannino ${ }^{50 a, 50 b}$, A. Sansoni ${ }^{47}$, C. Santoni ${ }^{34}$, R. Santonico ${ }^{133 a, 133 b}$, H. Santos ${ }^{126 a}$, I. Santoyo Castillo ${ }^{149}$, K. Sapp ${ }^{125}$, A. Sapronov ${ }^{65}$, J.G. Saraiva ${ }^{126 a, 126 d}$, B. Sarrazin ${ }^{21}$, O. Sasaki ${ }^{66}$, Y. Sasaki ${ }^{155}$, K. Sato ${ }^{160}$, G. Sauvage ${ }^{5, *}$, E. Sauvan ${ }^{5}$, G. Savage ${ }^{77}$, P. Savard ${ }^{158, d}$, C. Sawyer ${ }^{131}$, L. Sawyer ${ }^{79, p}$, J. Saxon ${ }^{31}$, C. Sbarra ${ }^{20 a}$, A. Sbrizzi ${ }^{20 a, 20 b}$, T. Scanlon ${ }^{78}$, D.A. Scannicchio ${ }^{163}$, M. Scarcella ${ }^{150}$, V. Scarfone ${ }^{37 a, 37 b}$, 
J. Schaarschmidt ${ }^{172}$, P. Schacht ${ }^{101}$, D. Schaefer ${ }^{30}$, R. Schaefer ${ }^{42}$, J. Schaeffer ${ }^{83}$, S. Schaepe ${ }^{21}$, S. Schaetzel ${ }^{58 b}$, U. Schäfer ${ }^{83}$, A.C. Schaffer ${ }^{117}$, D. Schaile ${ }^{100}$, R.D. Schamberger ${ }^{148}$, V. Scharf ${ }^{58 a}$, V.A. Schegelsky ${ }^{123}$, D. Scheirich ${ }^{129}$, M. Schernau ${ }^{163}$, C. Schiavi ${ }^{50 a, 50 b}$, C. Schillo ${ }^{48}$, M. Schioppa ${ }^{37 a, 37 b}$, S. Schlenker ${ }^{30}$, K. Schmieden ${ }^{30}$, C. Schmitt ${ }^{83}$, S. Schmitt ${ }^{58 b}$, S. Schmitt ${ }^{42}$, S. Schmitz ${ }^{83}$, B. Schneider ${ }^{159 a}$, Y.J. Schnellbach ${ }^{74}$, U. Schnoor ${ }^{44}$, L. Schoeffel ${ }^{136}$, A. Schoening ${ }^{58 b}$, B.D. Schoenrock ${ }^{90}$, E. Schopf ${ }^{21}$, A.L.S. Schorlemmer ${ }^{54}$, M. Schott ${ }^{83}$, D. Schouten ${ }^{159 a}$, J. Schovancova ${ }^{8}$, S. Schramm ${ }^{49}$, M. Schreyer ${ }^{174}$, N. Schuh ${ }^{83}$, M.J. Schultens ${ }^{21}$, H.-C. Schultz-Coulon ${ }^{58 a}$, H. Schulz ${ }^{16}$, M. Schumacher ${ }^{48}$, B.A. Schumm ${ }^{137}$, Ph. Schune ${ }^{136}$, C. Schwanenberger ${ }^{84}$, A. Schwartzman ${ }^{143}$, T.A. Schwarz ${ }^{89}$, Ph. Schwegler ${ }^{101}$, H. Schweiger ${ }^{84}$, Ph. Schwemling ${ }^{136}$, R. Schwienhorst ${ }^{90}$, J. Schwindling ${ }^{136}$, T. Schwindt ${ }^{21}$, F.G. Sciacca ${ }^{17}$, E. Scifo ${ }^{117}$, G. Sciolla ${ }^{23}$, F. Scuri ${ }^{124 a, 124 b}$, F. Scutti ${ }^{21}$, J. Searcy ${ }^{89}$, G. Sedov ${ }^{42}$, E. Sedykh ${ }^{123}$, P. Seema ${ }^{21}$, S.C. Seidel ${ }^{105}$, A. Seiden ${ }^{137}$, F. Seifert ${ }^{128}$, J.M. Seixas ${ }^{24 a}$, G. Sekhniaidze ${ }^{104 a}$, K. Sekhon ${ }^{89}$, S.J. Sekula ${ }^{40}$, D.M. Seliverstov ${ }^{123, *}$, N. Semprini-Cesari ${ }^{20 a, 20 b}$, C. Serfon ${ }^{30}$, L. Serin ${ }^{117}$, L. Serkin ${ }^{164 a, 164 b}$, T. Serre ${ }^{85}$, M. Sessa ${ }^{134 a, 134 b}$, R. Seuster ${ }^{159 a}$, H. Severini ${ }^{113}$, T. Sfiligoj ${ }^{75}$, F. Sforza ${ }^{30}$, A. Sfyrla ${ }^{30}$, E. Shabalina ${ }^{54}$, M. Shamim ${ }^{116}$, L.Y. Shan ${ }^{33 a}$, R. Shang ${ }^{165}$, J.T. Shank ${ }^{22}$, M. Shapiro ${ }^{15}$, P.B. Shatalov ${ }^{97}$, K. Shaw ${ }^{164 a, 164 b}$, S.M. Shaw ${ }^{84}$, A. Shcherbakova ${ }^{146 a, 146 b}$, C.Y. Shehu ${ }^{149}$, P. Sherwood ${ }^{78}$, L. Shi $^{151, a h}$, S. Shimizu ${ }^{67}$, C.O. Shimmin ${ }^{163}$, M. Shimojima ${ }^{102}$, M. Shiyakova ${ }^{65}$, A. Shmeleva ${ }^{96}$, D. Shoaleh Saadi ${ }^{95}$, M.J. Shochet ${ }^{31}$, S. Shojaii ${ }^{91 a, 91 b}$, S. Shrestha ${ }^{111}$, E. Shulga ${ }^{98}$, M.A. Shupe ${ }^{7}$, P. Sicho ${ }^{127}$, P.E. Sidebo ${ }^{147}$, O. Sidiropoulou ${ }^{174}$, D. Sidorov ${ }^{114}$, A. Sidoti ${ }^{20 a, 20 b}$, F. Siegert ${ }^{44}$, Dj. Sijacki ${ }^{13}$, J. Silva ${ }^{126 a, 126 d}$, Y. Silver ${ }^{153}$, S.B. Silverstein ${ }^{146 a}$, V. Simak ${ }^{128}$, O. Simard ${ }^{5}$, Lj. Simic ${ }^{13}$, S. Simion ${ }^{117}$, E. Simioni ${ }^{83}$, B. Simmons ${ }^{78}$, D. Simon ${ }^{34}$, P. Sinervo ${ }^{158}$, N.B. Sinev ${ }^{116}$, M. Sioli ${ }^{20 a, 20 b}$, G. Siragusa ${ }^{174}$, A.N. Sisakyan ${ }^{65, *}$, S.Yu. Sivoklokov ${ }^{99}$, J. Sjölin ${ }^{146 a, 146 b}$, T.B. Sjursen ${ }^{14}$, M.B. Skinner ${ }^{72}$, H.P. Skottowe ${ }^{57}$, P. Skubic ${ }^{113}$, M. Slater ${ }^{18}$, T. Slavicek ${ }^{128}$, M. Slawinska ${ }^{107}$, K. Sliwa ${ }^{161}$, V. Smakhtin ${ }^{172}$, B.H. Smart ${ }^{46}$, L. Smestad ${ }^{14}$, S.Yu. Smirnov ${ }^{98}$, Y. Smirnov ${ }^{98}$, L.N. Smirnova ${ }^{99, a i}$, O. Smirnova ${ }^{81}$, M.N.K. Smith ${ }^{35}$, R.W. Smith ${ }^{35}$, M. Smizanska ${ }^{72}$, K. Smolek ${ }^{128}$, A.A. Snesarev ${ }^{96}$, G. Snidero ${ }^{76}$, S. Snyder ${ }^{25}$, R. Sobie ${ }^{169, l}$, F. Socher ${ }^{44}$, A. Soffer ${ }^{153}$, D.A. Soh ${ }^{151, a h}$, G. Sokhrannyi ${ }^{75}$, C.A. Solans ${ }^{30}$, M. Solar ${ }^{128}$, J. Solc ${ }^{128}$, E.Yu. Soldatov ${ }^{98}$, U. Soldevila ${ }^{167}$, A.A. Solodkov ${ }^{130}$, A. Soloshenko ${ }^{65}$, O.V. Solovyanov ${ }^{130}$, V. Solovyev ${ }^{123}$, P. Sommer ${ }^{48}$, H.Y. Song ${ }^{33 b, z}$, N. Soni ${ }^{1}$, A. Sood ${ }^{15}$, A. Sopczak ${ }^{128}$, B. Sopko ${ }^{128}$, V. Sopko ${ }^{128}$, V. Sorin ${ }^{12}$, D. Sosa ${ }^{58 b}$, M. Sosebee ${ }^{8}$, C.L. Sotiropoulou ${ }^{124 a, 124 b}$, R. Soualah ${ }^{164 a, 164 c}$, A.M. Soukharev ${ }^{109, c}$, D. South ${ }^{42}$, B.C. Sowden ${ }^{77}$, S. Spagnolo ${ }^{73 a, 73 b}$, M. Spalla ${ }^{124 a, 124 b}$, M. Spangenberg ${ }^{170}$, F. Spanò ${ }^{77}$, W.R. Spearman ${ }^{57}$, D. Sperlich ${ }^{16}$, F. Spettel ${ }^{101}$, R. Spighi ${ }^{20 a}$, G. Spigo $^{30}$, L.A. Spiller ${ }^{88}$, M. Spousta ${ }^{129}$, R.D. St. Denis ${ }^{53, *}$, A. Stabile ${ }^{91 a}$, S. Staerz ${ }^{30}$, J. Stahlman ${ }^{122}$, R. Stamen ${ }^{58 a}$, S. Stamm ${ }^{16}$, E. Stanecka ${ }^{39}$, C. Stanescu ${ }^{134 a}$, M. Stanescu-Bellu ${ }^{42}$, M.M. Stanitzki ${ }^{42}$, S. Stapnes ${ }^{119}$, E.A. Starchenko ${ }^{130}$, J. Stark ${ }^{55}$, P. Staroba ${ }^{127}$, P. Starovoitov ${ }^{58 a}$, R. Staszewskii ${ }^{39}$, P. Steinberg ${ }^{25}$, B. Stelzer ${ }^{142}$, H.J. Stelzer ${ }^{30}$, O. Stelzer-Chilton ${ }^{159 a}$, H. Stenzel ${ }^{52}$, G.A. Stewart ${ }^{53}$, J.A. Stillings ${ }^{21}$, M.C. Stockton ${ }^{87}$, M. Stoebe ${ }^{87}$, G. Stoicea ${ }^{26 b}$, P. Stolte ${ }^{54}$, S. Stonjek ${ }^{101}$, A.R. Stradling ${ }^{8}$, A. Straessner ${ }^{44}$, M.E. Stramaglia ${ }^{17}$, J. Strandberg ${ }^{147}$, S. Strandberg ${ }^{146 a, 146 b}$, A. Strandlie ${ }^{119}$, E. Strauss ${ }^{143}$, M. Strauss ${ }^{113}$, P. Strizenec ${ }^{144 b}$, R. Ströhmer ${ }^{174}$, D.M. Strom ${ }^{116}$, R. Stroynowski ${ }^{40}$, A. Strubig ${ }^{106}$, S.A. Stucci ${ }^{17}$, B. Stugu ${ }^{14}$, N.A. Styles ${ }^{42}$, D. $\mathrm{Su}^{143}$, J. Su ${ }^{125}$, R. Subramaniam ${ }^{79}$, A. Succurro ${ }^{12}$, S. Suchek ${ }^{58 a}$, Y. Sugaya ${ }^{118}$, M. Suk ${ }^{128}$, V.V. Sulin ${ }^{96}$, S. Sultansoy ${ }^{4 c}$, T. Sumida ${ }^{68}$, S. Sun ${ }^{57}$, X. Sun ${ }^{33 a}$, J.E. Sundermann ${ }^{48}$, K. Suruliz ${ }^{149}$, G. Susinno ${ }^{37 a, 37 b}$, M.R. Sutton ${ }^{149}$, S. Suzuki ${ }^{66}$, M. Svatos ${ }^{127}$, M. Swiatlowski ${ }^{31}$, I. Sykora ${ }^{144 a}$, T. Sykora ${ }^{129}$, D. Ta ${ }^{48}$, C. Taccini ${ }^{134 a, 134 b}$, K. Tackmann ${ }^{42}$, J. Taenzer ${ }^{158}$, A. Taffard ${ }^{163}$, R. Tafirout ${ }^{159 a}$, N. Taiblum ${ }^{153}$, H. Takai ${ }^{25}$, R. Takashima ${ }^{69}$, H. Takeda $^{67}$, T. Takeshita ${ }^{140}$, Y. Takubo ${ }^{66}$, M. Talby ${ }^{85}$, A.A. Talyshev ${ }^{109, c}$, J.Y.C. Tam ${ }^{174}$, K.G. Tan ${ }^{88}$, J. Tanaka ${ }^{155}$, R. Tanaka ${ }^{117}$, S. Tanaka ${ }^{66}$, B.B. Tannenwald ${ }^{111}$, S. Tapia Araya ${ }^{32 b}$, S. Tapprogge ${ }^{83}$, 


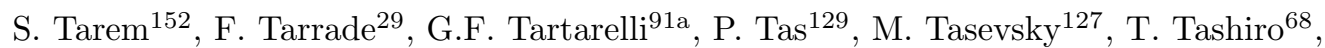
E. Tassi ${ }^{37 a, 37 b}$, A. Tavares Delgado ${ }^{126 a, 126 b}$, Y. Tayalati ${ }^{135 d}$, F.E. Taylor ${ }^{94}$, G.N. Taylor ${ }^{88}$, P.T.E. Taylor ${ }^{88}$, W. Taylor ${ }^{159 b}$, F.A. Teischinger ${ }^{30}$, M. Teixeira Dias Castanheira ${ }^{76}$, P. Teixeira-Dias ${ }^{77}$, K.K. Temming ${ }^{48}$, D. Temple ${ }^{142}$, H. Ten Kate ${ }^{30}$, P.K. Teng ${ }^{151}$, J.J. Teoh ${ }^{118}$, F. Tepel ${ }^{175}$, S. Terada ${ }^{66}$, K. Terashi ${ }^{155}$, J. Terron ${ }^{82}$, S. Terzo ${ }^{101}$, M. Testa ${ }^{47}$, R.J. Teuscher ${ }^{158, l}$, T. Theveneaux-Pelzer ${ }^{34}$, J.P. Thomas ${ }^{18}$, J. Thomas-Wilsker ${ }^{77}$, E.N. Thompson ${ }^{35}$, P.D. Thompson ${ }^{18}$, R.J. Thompson ${ }^{84}$, A.S. Thompson ${ }^{53}$, L.A. Thomsen ${ }^{176}$, E. Thomson ${ }^{122}$, M. Thomson ${ }^{28}$, R.P. Thun ${ }^{89, *}$, M.J. Tibbetts ${ }^{15}$, R.E. Ticse Torres ${ }^{85}$, V.O. Tikhomirov ${ }^{96, a j}$, Yu.A. Tikhonov ${ }^{109, c}$, S. Timoshenko ${ }^{98}$, E. Tiouchichine ${ }^{85}$, P. Tipton ${ }^{176}$, S. Tisserant ${ }^{85}$,

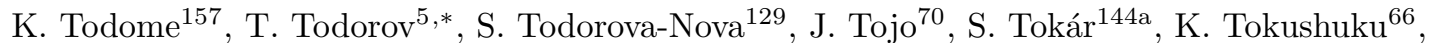
K. Tollefson ${ }^{90}$, E. Tolley ${ }^{57}$, L. Tomlinson ${ }^{84}$, M. Tomoto ${ }^{103}$, L. Tompkins ${ }^{143, a k}$, K. Toms ${ }^{105}$, E. Torrence ${ }^{116}$, H. Torres ${ }^{142}$, E. Torró Pastor ${ }^{138}$, J. Toth ${ }^{85, a l}$, F. Touchard ${ }^{85}$, D.R. Tovey ${ }^{139}$, T. Trefzger ${ }^{174}$, L. Tremblet ${ }^{30}$, A. Tricoli ${ }^{30}$, I.M. Trigger ${ }^{159 a}$, S. Trincaz-Duvoid ${ }^{80}$, M.F. Tripiana ${ }^{12}$, W. Trischuk ${ }^{158}$, B. Trocmé ${ }^{55}$, C. Troncon ${ }^{91 a}$, M. Trottier-McDonald ${ }^{15}$, M. Trovatelli $^{169}$, L. Truong ${ }^{164 a, 164 c}$, M. Trzebinski ${ }^{39}$, A. Trzupek ${ }^{39}$, C. Tsarouchas ${ }^{30}$, J.C-L. Tseng ${ }^{120}$, P.V. Tsiareshka ${ }^{92}$, D. Tsionou ${ }^{154}$, G. Tsipolitis ${ }^{10}$, N. Tsirintanis ${ }^{9}$, S. Tsiskaridze ${ }^{12}$, V. Tsiskaridze ${ }^{48}$, E.G. Tskhadadze ${ }^{51 a}$, K.M. Tsui ${ }^{60 a}$, I.I. Tsukerman ${ }^{97}$, V. Tsulaia ${ }^{15}$, S. Tsuno ${ }^{66}$, D. Tsybychev ${ }^{148}$, A. Tudorache ${ }^{26 b}$, V. Tudorache ${ }^{26 b}$, A.N. Tuna ${ }^{57}$, S.A. Tupputi ${ }^{20 a, 20 b}$,

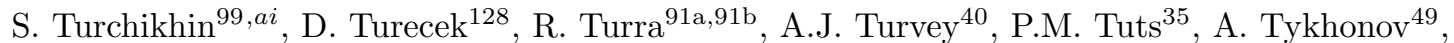
M. Tylmad ${ }^{146 a, 146 b}$, M. Tyndel ${ }^{131}$, I. Ueda ${ }^{155}$, R. Ueno ${ }^{29}$, M. Ughetto ${ }^{146 a, 146 b}$, F. Ukegawa ${ }^{160}$, G. Unal ${ }^{30}$, A. Undrus ${ }^{25}$, G. Unel ${ }^{163}$, F.C. Ungaro ${ }^{48}$, Y. Unno ${ }^{66}$, C. Unverdorben ${ }^{100}$, J. Urban ${ }^{144 b}$, P. Urquijo ${ }^{88}$, P. Urrejola ${ }^{83}$, G. Usai ${ }^{8}$, A. Usanova ${ }^{62}$, L. Vacavant ${ }^{85}$, V. Vacek ${ }^{128}$, B. Vachon ${ }^{87}$, C. Valderanis ${ }^{83}$, N. Valencic ${ }^{107}$, S. Valentinetti ${ }^{20 a, 20 b}$, A. Valero ${ }^{167}$, L. Valery ${ }^{12}$, S. Valkar ${ }^{129}$, S. Vallecorsa ${ }^{49}$, J.A. Valls Ferrer ${ }^{167}$, W. Van Den Wollenberg ${ }^{107}$, P.C. Van Der Deijl ${ }^{107}$, R. van der Geer ${ }^{107}$, H. van der Graaf ${ }^{107}$, N. van Eldik ${ }^{152}$, P. van Gemmeren ${ }^{6}$, J. Van Nieuwkoop ${ }^{142}$, I. van Vulpen ${ }^{107}$, M.C. van Woerden $^{30}$, M. Vanadia ${ }^{132 a, 132 b}$, W. Vandelli ${ }^{30}$,

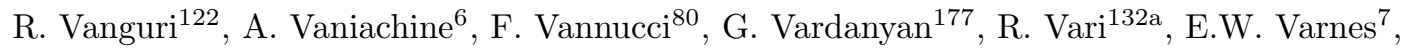

T. $\operatorname{Varol}^{40}$, D. Varouchas ${ }^{80}$, A. Vartapetian ${ }^{8}$, K.E. Varvell ${ }^{150}$, F. Vazeille ${ }^{34}$,

T. Vazquez Schroeder ${ }^{87}$, J. Veatch ${ }^{7}$, L.M. Veloce ${ }^{158}$, F. Veloso ${ }^{126 a, 126 c}$, T. Velz ${ }^{21}$,

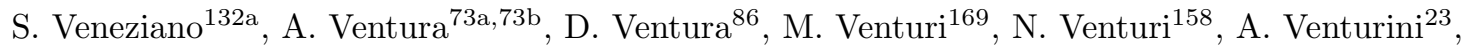
V. Vercesi ${ }^{121 a}$, M. Verducci ${ }^{132 a, 132 b}$, W. Verkerke ${ }^{107}$, J.C. Vermeulen ${ }^{107}$, A. Vest ${ }^{44}$, M.C. Vetterli ${ }^{142, d}$, O. Viazlo ${ }^{81}$, I. Vichou ${ }^{165}$, T. Vickey ${ }^{139}$, O.E. Vickey Boeriu ${ }^{139}$, G.H.A. Viehhauser ${ }^{120}$, S. Viel ${ }^{15}$, R. Vigne ${ }^{62}$, M. Villa ${ }^{20 a, 20 b}$, M. Villaplana Perez ${ }^{91 a, 91 b}$, E. Vilucchi ${ }^{47}$, M.G. Vincter ${ }^{29}$, V.B. Vinogradov ${ }^{65}$, I. Vivarelli ${ }^{149}$, F. Vives Vaque ${ }^{3}$, S. Vlachos $^{10}$, D. Vladoiu ${ }^{100}$, M. Vlasak ${ }^{128}$, M. Vogel ${ }^{32 a}$, P. Vokac $^{128}$, G. Volpi ${ }^{124 a, 124 b}$, M. Volpi ${ }^{88}$, H. von der Schmitt ${ }^{101}$, H. von Radziewski ${ }^{48}$, E. von Toerne ${ }^{21}$, V. Vorobel $^{129}$, K. Vorobev $^{98}$, M. $\operatorname{Vos}^{167}$, R. $\operatorname{Voss}^{30}$, J.H. Vossebeld ${ }^{74}$, N. Vranjes ${ }^{13}$, M. Vranjes Milosavljevic ${ }^{13}$, V. Vrba ${ }^{127}$, M. Vreeswijk ${ }^{107}$, R. Vuillermet ${ }^{30}$, I. Vukotic ${ }^{31}$, Z. Vykydal ${ }^{128}$, P. Wagner $^{21}$, W. Wagner ${ }^{175}$, H. Wahlberg ${ }^{71}$, S. Wahrmund ${ }^{44}$, J. Wakabayashi ${ }^{103}$, J. Walder ${ }^{72}$, R. Walker ${ }^{100}$, W. Walkowiak ${ }^{141}$, C. Wang ${ }^{151}$, F. Wang ${ }^{173}$, H. Wang ${ }^{15}$, H. Wang ${ }^{40}$, J. Wang ${ }^{42}$, J. Wang ${ }^{150}$, K. Wang ${ }^{87}$, R. Wang ${ }^{6}$, S.M. Wang ${ }^{151}$, T. Wang ${ }^{21}$, T. Wang ${ }^{35}$, X. Wang ${ }^{176}$, C. Wanotayaroj ${ }^{116}$, A. Warburton ${ }^{87}$, C.P. Ward ${ }^{28}$, D.R. Wardrope ${ }^{78}$, A. Washbrook ${ }^{46}$, C. Wasicki ${ }^{42}$, P.M. Watkins ${ }^{18}$, A.T. Watson ${ }^{18}$, I.J. Watson ${ }^{150}$, M.F. Watson ${ }^{18}$, G. Watts ${ }^{138}$, S. Watts ${ }^{84}$, B.M. Waugh ${ }^{78}$, S. Webb ${ }^{84}$,

M.S. Weber ${ }^{17}$, S.W. Weber ${ }^{174}$, J.S. Webster ${ }^{31}$, A.R. Weidberg ${ }^{120}$, B. Weinert ${ }^{61}$, J. Weingarten ${ }^{54}$, C. Weiser ${ }^{48}$, H. Weits ${ }^{107}$, P.S. Wells ${ }^{30}$, T. Wenaus ${ }^{25}$, T. Wengler ${ }^{30}$, S. Wenig ${ }^{30}$, N. Wermes ${ }^{21}$, M. Werner ${ }^{48}$, P. Werner ${ }^{30}$, M. Wessels ${ }^{58 a}$, J. Wetter ${ }^{161}$, K. Whalen ${ }^{116}$, A.M. Wharton ${ }^{72}$, A. White ${ }^{8}$, M.J. White ${ }^{1}$, R. White ${ }^{32 b}$, S. White ${ }^{124 a, 124 b}$, D. Whiteson ${ }^{163}$, F.J. Wickens ${ }^{131}$, W. Wiedenmann ${ }^{173}$, M. Wielers ${ }^{131}$, P. Wienemann ${ }^{21}$, C. Wiglesworth ${ }^{36}$, L.A.M. Wiik-Fuchs ${ }^{21}$, 
A. Wildauer ${ }^{101}$, H.G. Wilkens ${ }^{30}$, H.H. Williams ${ }^{122}$, S. Williams ${ }^{107}$, C. Willis ${ }^{90}$, S. Willocq ${ }^{86}$, A. Wilson ${ }^{89}$, J.A. Wilson ${ }^{18}$, I. Wingerter-Seez ${ }^{5}$, F. Winklmeier ${ }^{116}$, B.T. Winter ${ }^{21}$, M. Wittgen ${ }^{143}$, J. Wittkowski ${ }^{100}$, S.J. Wollstadt ${ }^{83}$, M.W. Wolter ${ }^{39}$, H. Wolters ${ }^{126 a, 126 c}$, B.K. Wosiek ${ }^{39}$, J. Wotschack ${ }^{30}$, M.J. Woudstra ${ }^{84}$, K.W. Wozniak ${ }^{39}$, M. Wu ${ }^{55}$, M. Wu ${ }^{31}$, S.L. Wu ${ }^{173}$, X. Wu ${ }^{49}$, Y. $\mathrm{Wu}^{89}$, T.R. Wyatt ${ }^{84}$, B.M. Wynne ${ }^{46}$, S. Xella ${ }^{36}$, D. Xu ${ }^{33 a}$, L. Xu ${ }^{25}$, B. Yabsley ${ }^{150}$, S. Yacoob ${ }^{145 a}$, R. Yakabe ${ }^{67}$, M. Yamada ${ }^{66}$, D. Yamaguchi ${ }^{157}$, Y. Yamaguchi ${ }^{118}$, A. Yamamoto ${ }^{66}$, S. Yamamoto ${ }^{155}$, T. Yamanaka ${ }^{155}$, K. Yamauchi ${ }^{103}$, Y. Yamazaki ${ }^{67}$, Z. Yan ${ }^{22}$, H. Yang ${ }^{33 e}$, H. Yang ${ }^{173}$, Y. Yang ${ }^{151}$, W-M. Yao ${ }^{15}$, Y.C. Yap ${ }^{80}$, Y. Yasu ${ }^{66}$, E. Yatsenko ${ }^{5}$, K.H. Yau Wong ${ }^{21}$, J. $\mathrm{Ye}^{40}$, S. $\mathrm{Ye}^{25}$, I. Yeletskikh ${ }^{65}$, A.L. Yen ${ }^{57}$, E. Yildirim ${ }^{4}$, K. Yorita ${ }^{171}$, R. Yoshida ${ }^{6}$, K. Yoshihara ${ }^{122}$, C. Young ${ }^{143}$, C.J.S. Young ${ }^{30}$, S. Youssef ${ }^{22}$, D.R. $\mathrm{Yu}^{15}$, J. $_{\mathrm{Yu}^{8}}$, J.M. Yu ${ }^{89}$, J. $\mathrm{Yu}^{114}$, L. Yuan ${ }^{67}$, S.P.Y. Yuen ${ }^{21}$, A. Yurkewicz ${ }^{108}$, I. Yusuff ${ }^{28, a m}$, B. Zabinski ${ }^{39}$, R. Zaidan ${ }^{63}$, A.M. Zaitsev ${ }^{130, a d}$, J. Zalieckas ${ }^{14}$, A. Zaman ${ }^{148}$, S. Zambito ${ }^{57}$, L. Zanello ${ }^{132 a, 132 b}$, D. Zanzi ${ }^{88}$, C. Zeitnitz ${ }^{175}$, M. Zeman ${ }^{128}$, A. Zemla ${ }^{38 a}$, J.C. Zeng ${ }^{165}$, Q. Zeng ${ }^{143}$, K. Zengel ${ }^{23}$, O. Zenin ${ }^{130}$, T. Ženis ${ }^{144 a}$, D. Zerwas ${ }^{117}$, D. Zhang ${ }^{89}$, F. Zhang ${ }^{173}$, G. Zhang ${ }^{33 b}$, H. Zhang ${ }^{33 c}$, J. Zhang ${ }^{6}$, L. Zhang ${ }^{48}$, R. Zhang ${ }^{33 b, j}$, X. Zhang ${ }^{33 d}$, Z. Zhang ${ }^{117}$, X. Zhao ${ }^{40}$, Y. Zhao ${ }^{33 d, 117}$, Z. Zhao ${ }^{33 b}$, A. Zhemchugov ${ }^{65}$, J. Zhong ${ }^{120}$, B. Zhou ${ }^{89}$, C. Zhou ${ }^{45}$, L. Zhou ${ }^{35}$, L. Zhou ${ }^{40}$, M. Zhou ${ }^{148}$, N. Zhou ${ }^{33 f}$, C.G. Zhu ${ }^{33 d}$, H. Zhu ${ }^{33 a}$, J. Zhu ${ }^{89}$, Y. Zhu ${ }^{33 b}$, X. Zhuang ${ }^{33 a}$, K. Zhukov ${ }^{96}$, A. Zibell ${ }^{174}$, D. Zieminska ${ }^{61}$, N.I. Zimine ${ }^{65}$, C. Zimmermann ${ }^{83}$, S. Zimmermann ${ }^{48}$, Z. Zinonos ${ }^{54}$, M. Zinser ${ }^{83}$, M. Ziolkowski ${ }^{141}$, L. Živković ${ }^{13}$, G. Zobernig ${ }^{173}$, A. Zoccoli ${ }^{20 a, 20 b}$, M. zur Nedden ${ }^{16}$, G. Zurzolo ${ }^{104 a, 104 b}$, L. Zwalinski ${ }^{30}$

1 Department of Physics, University of Adelaide, Adelaide, Australia

2 Physics Department, SUNY Albany, Albany NY, United States of America

3 Department of Physics, University of Alberta, Edmonton AB, Canada

4 (a) Department of Physics, Ankara University, Ankara; ${ }^{(b)}$ Istanbul Aydin University, Istanbul; ${ }^{(c)}$ Division of Physics, TOBB University of Economics and Technology, Ankara, Turkey

5 LAPP, CNRS/IN2P3 and Université Savoie Mont Blanc, Annecy-le-Vieux, France

6 High Energy Physics Division, Argonne National Laboratory, Argonne IL, United States of America

7 Department of Physics, University of Arizona, Tucson AZ, United States of America

8 Department of Physics, The University of Texas at Arlington, Arlington TX, United States of America

9 Physics Department, University of Athens, Athens, Greece

10 Physics Department, National Technical University of Athens, Zografou, Greece

11 Institute of Physics, Azerbaijan Academy of Sciences, Baku, Azerbaijan

12 Institut de Física d'Altes Energies and Departament de Física de la Universitat Autònoma de Barcelona, Barcelona, Spain

13 Institute of Physics, University of Belgrade, Belgrade, Serbia

14 Department for Physics and Technology, University of Bergen, Bergen, Norway

15 Physics Division, Lawrence Berkeley National Laboratory and University of California, Berkeley CA, United States of America

16 Department of Physics, Humboldt University, Berlin, Germany

17 Albert Einstein Center for Fundamental Physics and Laboratory for High Energy Physics, University of Bern, Bern, Switzerland

18 School of Physics and Astronomy, University of Birmingham, Birmingham, United Kingdom

19 (a) Department of Physics, Bogazici University, Istanbul; ${ }^{(b)}$ Department of Physics Engineering, Gaziantep University, Gaziantep; ${ }^{(c)}$ Department of Physics, Dogus University, Istanbul, Turkey

20 (a) INFN Sezione di Bologna; ${ }^{(b)}$ Dipartimento di Fisica e Astronomia, Università di Bologna, Bologna, Italy

21 Physikalisches Institut, University of Bonn, Bonn, Germany

22 Department of Physics, Boston University, Boston MA, United States of America

23 Department of Physics, Brandeis University, Waltham MA, United States of America 
24 (a) Universidade Federal do Rio De Janeiro COPPE/EE/IF, Rio de Janeiro; ${ }^{(b)}$ Electrical Circuits Department, Federal University of Juiz de Fora (UFJF), Juiz de Fora; ${ }^{(c)}$ Federal University of Sao Joao del Rei (UFSJ), Sao Joao del Rei; ${ }^{(d)}$ Instituto de Fisica, Universidade de Sao Paulo, Sao Paulo, Brazil

25 Physics Department, Brookhaven National Laboratory, Upton NY, United States of America

26 (a) Transilvania University of Brasov, Brasov; ${ }^{(b)}$ National Institute of Physics and Nuclear Engineering, Bucharest; ${ }^{(c)}$ National Institute for Research and Development of Isotopic and Molecular Technologies, Physics Department, Cluj Napoca; ${ }^{(d)}$ University Politehnica Bucharest, Bucharest; ${ }^{(e)}$ West University in Timisoara, Timisoara, Romania

27 Departamento de Física, Universidad de Buenos Aires, Buenos Aires, Argentina

28 Cavendish Laboratory, University of Cambridge, Cambridge, United Kingdom

29 Department of Physics, Carleton University, Ottawa ON, Canada

30 CERN, Geneva, Switzerland

31 Enrico Fermi Institute, University of Chicago, Chicago IL, United States of America

32 (a) Departamento de Física, Pontificia Universidad Católica de Chile, Santiago; ${ }^{(b)}$ Departamento de Física, Universidad Técnica Federico Santa María, Valparaíso, Chile

33 (a) Institute of High Energy Physics, Chinese Academy of Sciences, Beijing; ${ }^{(b)}$ Department of Modern Physics, University of Science and Technology of China, Anhui; (c) Department of Physics, Nanjing University, Jiangsu; ${ }^{(d)}$ School of Physics, Shandong University, Shandong; ${ }^{(e)}$ Department of Physics and Astronomy, Shanghai Key Laboratory for Particle Physics and Cosmology, Shanghai Jiao Tong University, Shanghai; ${ }^{(f)}$ Physics Department, Tsinghua University, Beijing 100084, China

34 Laboratoire de Physique Corpusculaire, Clermont Université and Université Blaise Pascal and CNRS/IN2P3, Clermont-Ferrand, France

35 Nevis Laboratory, Columbia University, Irvington NY, United States of America

36 Niels Bohr Institute, University of Copenhagen, Kobenhavn, Denmark

37 (a) INFN Gruppo Collegato di Cosenza, Laboratori Nazionali di Frascati; ${ }^{(b)}$ Dipartimento di Fisica, Università della Calabria, Rende, Italy

38 (a) AGH University of Science and Technology, Faculty of Physics and Applied Computer Science, Krakow; ${ }^{(b)}$ Marian Smoluchowski Institute of Physics, Jagiellonian University, Krakow, Poland

39 Institute of Nuclear Physics Polish Academy of Sciences, Krakow, Poland

40 Physics Department, Southern Methodist University, Dallas TX, United States of America

41 Physics Department, University of Texas at Dallas, Richardson TX, United States of America

42 DESY, Hamburg and Zeuthen, Germany

43 Institut für Experimentelle Physik IV, Technische Universität Dortmund, Dortmund, Germany

44 Institut für Kern- und Teilchenphysik, Technische Universität Dresden, Dresden, Germany

45 Department of Physics, Duke University, Durham NC, United States of America

46 SUPA - School of Physics and Astronomy, University of Edinburgh, Edinburgh, United Kingdom

47 INFN Laboratori Nazionali di Frascati, Frascati, Italy

48 Fakultät für Mathematik und Physik, Albert-Ludwigs-Universität, Freiburg, Germany

49 Section de Physique, Université de Genève, Geneva, Switzerland

50 (a) INFN Sezione di Genova; ${ }^{(b)}$ Dipartimento di Fisica, Università di Genova, Genova, Italy

51 (a) E. Andronikashvili Institute of Physics, Iv. Javakhishvili Tbilisi State University, Tbilisi; ${ }^{(b)}$ High Energy Physics Institute, Tbilisi State University, Tbilisi, Georgia

52 II Physikalisches Institut, Justus-Liebig-Universität Giessen, Giessen, Germany

53 SUPA - School of Physics and Astronomy, University of Glasgow, Glasgow, United Kingdom

54 II Physikalisches Institut, Georg-August-Universität, Göttingen, Germany

55 Laboratoire de Physique Subatomique et de Cosmologie, Université Grenoble-Alpes, CNRS/IN2P3, Grenoble, France

56 Department of Physics, Hampton University, Hampton VA, United States of America

57 Laboratory for Particle Physics and Cosmology, Harvard University, Cambridge MA, United States of America 
58 (a) Kirchhoff-Institut für Physik, Ruprecht-Karls-Universität Heidelberg, Heidelberg; ${ }^{(b)}$ Physikalisches Institut, Ruprecht-Karls-Universität Heidelberg, Heidelberg; ${ }^{(c)}$ ZITI Institut für technische Informatik, Ruprecht-Karls-Universität Heidelberg, Mannheim, Germany

59 Faculty of Applied Information Science, Hiroshima Institute of Technology, Hiroshima, Japan

60 (a) Department of Physics, The Chinese University of Hong Kong, Shatin, N.T., Hong Kong; ${ }^{(b)}$ Department of Physics, The University of Hong Kong, Hong Kong; ${ }^{(c)}$ Department of Physics, The Hong Kong University of Science and Technology, Clear Water Bay, Kowloon, Hong Kong, China

61 Department of Physics, Indiana University, Bloomington IN, United States of America

62 Institut für Astro- und Teilchenphysik, Leopold-Franzens-Universität, Innsbruck, Austria

63 University of Iowa, Iowa City IA, United States of America

64 Department of Physics and Astronomy, Iowa State University, Ames IA, United States of America

65 Joint Institute for Nuclear Research, JINR Dubna, Dubna, Russia

66 KEK, High Energy Accelerator Research Organization, Tsukuba, Japan

67 Graduate School of Science, Kobe University, Kobe, Japan

68 Faculty of Science, Kyoto University, Kyoto, Japan

69 Kyoto University of Education, Kyoto, Japan

70 Department of Physics, Kyushu University, Fukuoka, Japan

71 Instituto de Física La Plata, Universidad Nacional de La Plata and CONICET, La Plata, Argentina

72 Physics Department, Lancaster University, Lancaster, United Kingdom

73 (a) INFN Sezione di Lecce; ${ }^{(b)}$ Dipartimento di Matematica e Fisica, Università del Salento, Lecce, Italy

74 Oliver Lodge Laboratory, University of Liverpool, Liverpool, United Kingdom

75 Department of Physics, Jožef Stefan Institute and University of Ljubljana, Ljubljana, Slovenia

76 School of Physics and Astronomy, Queen Mary University of London, London, United Kingdom

77 Department of Physics, Royal Holloway University of London, Surrey, United Kingdom

78 Department of Physics and Astronomy, University College London, London, United Kingdom

79 Louisiana Tech University, Ruston LA, United States of America

80 Laboratoire de Physique Nucléaire et de Hautes Energies, UPMC and Université Paris-Diderot and CNRS/IN2P3, Paris, France

81 Fysiska institutionen, Lunds universitet, Lund, Sweden

82 Departamento de Fisica Teorica C-15, Universidad Autonoma de Madrid, Madrid, Spain

83 Institut für Physik, Universität Mainz, Mainz, Germany

84 School of Physics and Astronomy, University of Manchester, Manchester, United Kingdom

85 CPPM, Aix-Marseille Université and CNRS/IN2P3, Marseille, France

86 Department of Physics, University of Massachusetts, Amherst MA, United States of America

87 Department of Physics, McGill University, Montreal QC, Canada

88 School of Physics, University of Melbourne, Victoria, Australia

89 Department of Physics, The University of Michigan, Ann Arbor MI, United States of America

90 Department of Physics and Astronomy, Michigan State University, East Lansing MI, United States of America

91 (a) INFN Sezione di Milano; ${ }^{(b)}$ Dipartimento di Fisica, Università di Milano, Milano, Italy

92 B.I. Stepanov Institute of Physics, National Academy of Sciences of Belarus, Minsk, Republic of Belarus

93 National Scientific and Educational Centre for Particle and High Energy Physics, Minsk, Republic of Belarus

94 Department of Physics, Massachusetts Institute of Technology, Cambridge MA, United States of America

95 Group of Particle Physics, University of Montreal, Montreal QC, Canada

96 P.N. Lebedev Institute of Physics, Academy of Sciences, Moscow, Russia

97 Institute for Theoretical and Experimental Physics (ITEP), Moscow, Russia

98 National Research Nuclear University MEPhI, Moscow, Russia 
D.V. Skobeltsyn Institute of Nuclear Physics, M.V. Lomonosov Moscow State University, Moscow, Russia

100 Fakultät für Physik, Ludwig-Maximilians-Universität München, München, Germany

101 Max-Planck-Institut für Physik (Werner-Heisenberg-Institut), München, Germany

102 Nagasaki Institute of Applied Science, Nagasaki, Japan

103 Graduate School of Science and Kobayashi-Maskawa Institute, Nagoya University, Nagoya, Japan

104 (a) INFN Sezione di Napoli; ${ }^{(b)}$ Dipartimento di Fisica, Università di Napoli, Napoli, Italy

105 Department of Physics and Astronomy, University of New Mexico, Albuquerque NM, United States of America

106 Institute for Mathematics, Astrophysics and Particle Physics, Radboud University Nijmegen/Nikhef, Nijmegen, Netherlands

107 Nikhef National Institute for Subatomic Physics and University of Amsterdam, Amsterdam, Netherlands

108 Department of Physics, Northern Illinois University, DeKalb IL, United States of America

109 Budker Institute of Nuclear Physics, SB RAS, Novosibirsk, Russia

110 Department of Physics, New York University, New York NY, United States of America

111 Ohio State University, Columbus OH, United States of America

112 Faculty of Science, Okayama University, Okayama, Japan

113 Homer L. Dodge Department of Physics and Astronomy, University of Oklahoma, Norman OK, United States of America

114 Department of Physics, Oklahoma State University, Stillwater OK, United States of America

115 Palacký University, RCPTM, Olomouc, Czech Republic

116 Center for High Energy Physics, University of Oregon, Eugene OR, United States of America

117 LAL, Université Paris-Sud and CNRS/IN2P3, Orsay, France

118 Graduate School of Science, Osaka University, Osaka, Japan

119 Department of Physics, University of Oslo, Oslo, Norway

120 Department of Physics, Oxford University, Oxford, United Kingdom

$121{ }^{(a)}$ INFN Sezione di Pavia; ${ }^{(b)}$ Dipartimento di Fisica, Università di Pavia, Pavia, Italy

122 Department of Physics, University of Pennsylvania, Philadelphia PA, United States of America

123 National Research Centre 'Kurchatov Institute' B.P.Konstantinov Petersburg Nuclear Physics Institute, St. Petersburg, Russia

124 (a) INFN Sezione di Pisa; ${ }^{(b)}$ Dipartimento di Fisica E. Fermi, Università di Pisa, Pisa, Italy

125 Department of Physics and Astronomy, University of Pittsburgh, Pittsburgh PA, United States of America

126 (a) Laboratório de Instrumentação e Física Experimental de Partículas - LIP, Lisboa; ${ }^{(b)}$ Faculdade de Ciências, Universidade de Lisboa, Lisboa; ( ${ }^{(c)}$ Department of Physics, University of Coimbra, Coimbra; ${ }^{(d)}$ Centro de Física Nuclear da Universidade de Lisboa, Lisboa; ${ }^{(e)}$ Departamento de Fisica, Universidade do Minho, Braga; ${ }^{(f)}$ Departamento de Fisica Teorica y del Cosmos and CAFPE, Universidad de Granada, Granada (Spain); ${ }^{(g)}$ Dep Fisica and CEFITEC of Faculdade de Ciencias e Tecnologia, Universidade Nova de Lisboa, Caparica, Portugal

127 Institute of Physics, Academy of Sciences of the Czech Republic, Praha, Czech Republic

128 Czech Technical University in Prague, Praha, Czech Republic

129 Faculty of Mathematics and Physics, Charles University in Prague, Praha, Czech Republic

130 State Research Center Institute for High Energy Physics (Protvino), NRC KI, Russia

131 Particle Physics Department, Rutherford Appleton Laboratory, Didcot, United Kingdom

132 (a) INFN Sezione di Roma; ${ }^{(b)}$ Dipartimento di Fisica, Sapienza Università di Roma, Roma, Italy

133 (a) INFN Sezione di Roma Tor Vergata; ${ }^{(b)}$ Dipartimento di Fisica, Università di Roma Tor Vergata, Roma, Italy

134 (a) INFN Sezione di Roma Tre; ${ }^{(b)}$ Dipartimento di Matematica e Fisica, Università Roma Tre, Roma, Italy

135 (a) Faculté des Sciences Ain Chock, Réseau Universitaire de Physique des Hautes Energies Université Hassan II, Casablanca; ${ }^{(b)}$ Centre National de l'Energie des Sciences Techniques Nucleaires, Rabat; ${ }^{(c)}$ Faculté des Sciences Semlalia, Université Cadi Ayyad, LPHEA-Marrakech; 
(d) Faculté des Sciences, Université Mohamed Premier and LPTPM, Oujda; ${ }^{(e)}$ Faculté des sciences, Université Mohammed V, Rabat, Morocco

136 DSM/IRFU (Institut de Recherches sur les Lois Fondamentales de l'Univers), CEA Saclay (Commissariat à l'Energie Atomique et aux Energies Alternatives), Gif-sur-Yvette, France

137 Santa Cruz Institute for Particle Physics, University of California Santa Cruz, Santa Cruz CA, United States of America

138 Department of Physics, University of Washington, Seattle WA, United States of America

139 Department of Physics and Astronomy, University of Sheffield, Sheffield, United Kingdom

140 Department of Physics, Shinshu University, Nagano, Japan

141 Fachbereich Physik, Universität Siegen, Siegen, Germany

142 Department of Physics, Simon Fraser University, Burnaby BC, Canada

143 SLAC National Accelerator Laboratory, Stanford CA, United States of America

144 (a) Faculty of Mathematics, Physics 8 Informatics, Comenius University, Bratislava; ${ }^{(b)}$

Department of Subnuclear Physics, Institute of Experimental Physics of the Slovak Academy of Sciences, Kosice, Slovak Republic

145 (a) Department of Physics, University of Cape Town, Cape Town; ${ }^{(b)}$ Department of Physics, University of Johannesburg, Johannesburg; ${ }^{(c)}$ School of Physics, University of the Witwatersrand, Johannesburg, South Africa

146 (a) Department of Physics, Stockholm University; ${ }^{(b)}$ The Oskar Klein Centre, Stockholm, Sweden 147 Physics Department, Royal Institute of Technology, Stockholm, Sweden

148 Departments of Physics 83 Astronomy and Chemistry, Stony Brook University, Stony Brook NY, United States of America

149 Department of Physics and Astronomy, University of Sussex, Brighton, United Kingdom

150 School of Physics, University of Sydney, Sydney, Australia

151 Institute of Physics, Academia Sinica, Taipei, Taiwan

152 Department of Physics, Technion: Israel Institute of Technology, Haifa, Israel

153 Raymond and Beverly Sackler School of Physics and Astronomy, Tel Aviv University, Tel Aviv, Israel

154 Department of Physics, Aristotle University of Thessaloniki, Thessaloniki, Greece

155 International Center for Elementary Particle Physics and Department of Physics, The University of Tokyo, Tokyo, Japan

156 Graduate School of Science and Technology, Tokyo Metropolitan University, Tokyo, Japan

157 Department of Physics, Tokyo Institute of Technology, Tokyo, Japan

158 Department of Physics, University of Toronto, Toronto ON, Canada

159 (a) TRIUMF, Vancouver BC; ${ }^{(b)}$ Department of Physics and Astronomy, York University, Toronto ON, Canada

160 Faculty of Pure and Applied Sciences, and Center for Integrated Research in Fundamental Science and Engineering, University of Tsukuba, Tsukuba, Japan

161 Department of Physics and Astronomy, Tufts University, Medford MA, United States of America

162 Centro de Investigaciones, Universidad Antonio Narino, Bogota, Colombia

163 Department of Physics and Astronomy, University of California Irvine, Irvine CA, United States of America

164 (a) INFN Gruppo Collegato di Udine, Sezione di Trieste, Udine; ${ }^{(b)}$ ICTP, Trieste; ${ }^{(c)}$ Dipartimento di Chimica, Fisica e Ambiente, Università di Udine, Udine, Italy

165 Department of Physics, University of Illinois, Urbana IL, United States of America

166 Department of Physics and Astronomy, University of Uppsala, Uppsala, Sweden

167 Instituto de Física Corpuscular (IFIC) and Departamento de Física Atómica, Molecular y Nuclear and Departamento de Ingeniería Electrónica and Instituto de Microelectrónica de Barcelona (IMB-CNM), University of Valencia and CSIC, Valencia, Spain

168 Department of Physics, University of British Columbia, Vancouver BC, Canada

169 Department of Physics and Astronomy, University of Victoria, Victoria BC, Canada

170 Department of Physics, University of Warwick, Coventry, United Kingdom 
171 Waseda University, Tokyo, Japan

172 Department of Particle Physics, The Weizmann Institute of Science, Rehovot, Israel

173 Department of Physics, University of Wisconsin, Madison WI, United States of America

174 Fakultät für Physik und Astronomie, Julius-Maximilians-Universität, Würzburg, Germany

175 Fachbereich C Physik, Bergische Universität Wuppertal, Wuppertal, Germany

176 Department of Physics, Yale University, New Haven CT, United States of America

177 Yerevan Physics Institute, Yerevan, Armenia

178 Centre de Calcul de l'Institut National de Physique Nucléaire et de Physique des Particules (IN2P3), Villeurbanne, France

a Also at Department of Physics, King's College London, London, United Kingdom

${ }^{b}$ Also at Institute of Physics, Azerbaijan Academy of Sciences, Baku, Azerbaijan

c Also at Novosibirsk State University, Novosibirsk, Russia

${ }^{d}$ Also at TRIUMF, Vancouver BC, Canada

e Also at Department of Physics $\&$ Astronomy, University of Louisville, Louisville, KY, United States of America

${ }^{f}$ Also at Department of Physics, California State University, Fresno CA, United States of America

$g$ Also at Department of Physics, University of Fribourg, Fribourg, Switzerland

${ }^{h}$ Also at Departamento de Fisica e Astronomia, Faculdade de Ciencias, Universidade do Porto, Portugal

${ }^{i}$ Also at Tomsk State University, Tomsk, Russia

j Also at CPPM, Aix-Marseille Université and CNRS/IN2P3, Marseille, France

${ }^{k}$ Also at Universita di Napoli Parthenope, Napoli, Italy

${ }^{l}$ Also at Institute of Particle Physics (IPP), Canada

${ }^{m}$ Also at Particle Physics Department, Rutherford Appleton Laboratory, Didcot, United Kingdom

$n$ Also at Department of Physics, St. Petersburg State Polytechnical University, St. Petersburg, Russia

o Also at Department of Physics, The University of Michigan, Ann Arbor MI, United States of America

${ }^{p}$ Also at Louisiana Tech University, Ruston LA, United States of America

$q$ Also at Institucio Catalana de Recerca i Estudis Avancats, ICREA, Barcelona, Spain

$r$ Also at Graduate School of Science, Osaka University, Osaka, Japan

$s$ Also at Department of Physics, National Tsing Hua University, Taiwan

${ }^{t}$ Also at Department of Physics, The University of Texas at Austin, Austin TX, United States of America

${ }^{u}$ Also at Institute of Theoretical Physics, Ilia State University, Tbilisi, Georgia

$\checkmark$ Also at CERN, Geneva, Switzerland

w Also at Georgian Technical University (GTU), Tbilisi, Georgia

$x$ Also at Manhattan College, New York NY, United States of America

$y$ Also at Hellenic Open University, Patras, Greece

$z$ Also at Institute of Physics, Academia Sinica, Taipei, Taiwan

aa Also at LAL, Université Paris-Sud and CNRS/IN2P3, Orsay, France

ab Also at Academia Sinica Grid Computing, Institute of Physics, Academia Sinica, Taipei, Taiwan

ac Also at School of Physics, Shandong University, Shandong, China

ad Also at Moscow Institute of Physics and Technology State University, Dolgoprudny, Russia

ae Also at Section de Physique, Université de Genève, Geneva, Switzerland

af Also at International School for Advanced Studies (SISSA), Trieste, Italy

ag Also at Department of Physics and Astronomy, University of South Carolina, Columbia SC, United States of America

ah Also at School of Physics and Engineering, Sun Yat-sen University, Guangzhou, China

ai Also at Faculty of Physics, M.V.Lomonosov Moscow State University, Moscow, Russia

aj Also at National Research Nuclear University MEPhI, Moscow, Russia 
ak Also at Department of Physics, Stanford University, Stanford CA, United States of America

al Also at Institute for Particle and Nuclear Physics, Wigner Research Centre for Physics, Budapest, Hungary

am Also at University of Malaya, Department of Physics, Kuala Lumpur, Malaysia

* Deceased 